\title{
An implementation of Wang tilings for the representation of metallic glasses in Molecular Dynamics
}

\author{
by \\ Roxana Barcelo Singh \\ A thesis submitted to the \\ Faculty of Graduate and Postdoctoral Affairs \\ in partial fulfillment of the requirements for the degree of
}

Master of Applied Science in Materials Engineering

Ottawa-Carleton Institute for Mechanical and Aerospace Engineering

Department of Mechanical and Aerospace Engineering

Carleton University

Ottawa, Ontario

August, 2016

(C) Copyright

Roxana Barcelo Singh, 2016 
The undersigned hereby recommends to the

Faculty of Graduate and Postdoctoral Affairs

acceptance of the thesis

\title{
An implementation of Wang tilings for the representation of metallic glasses in Molecular Dynamics
}

\author{
submitted by Roxana Barcelo Singh \\ in partial fulfillment of the requirements for the degree of \\ Master of Applied Science in Materials Engineering
}

Dr. Ronald E. Miller, Supervisor

Dr. Ronald E. Miller, Department Chair

Carleton University

August, 2016 


\section{Abstract}

This thesis presents an implementation of a mathematical model known as Wang tilings for the representation of metallic glasses in atomistic simulations, specifically Molecular Dynamics. The purpose of this work is to assess whether a Wang tiling specimen can be considered an accurate representation of a metallic glass. Being able to implement Wang tilings for the representation of amorphous structures can potentially increase the size of the systems being studied and enable researchers to study small tile sets instead of large systems and achieve the same results.

A technique for the creation of a Wang tiling specimen and a true specimen, considered to be a metallic glass, is developed in this work. Once they are created, the mechanical response to uniaxial tension deformation for both specimens is compared and analyzed macroscopic and microscopically.

The macroscopic analysis consisted of comparing stress-strain curves which revealed that the Wang tiling specimen has a lower maximum strength compared to the true specimen. The microscopic analysis shed insights on the possible reasons for this difference and revealed that the Wang tiling specimen presents a higher concentration of atomic bond deficiency defects. It was also found that neither specimens presented shear band formation, but that shear was evenly distributed across the specimens.

While a technique for the creation of a Wang tiling specimen was accurately 
developed, the tiled system is not a surrogate of the true specimen. The value of this research is the adaptation of Wang tilings in Materials Engineering and is a first approach for their implementation. 


\section{Acknowledgments}

Special thanks to Dr. Ronald E. Miller for the opportunity to work on this project, his vision, guidance, encouragement, and his financial support.

Thanks to Dr. Martin Doškář from the University of Prague for his contributions on the development of Wang tilings.

Special thanks to Consejo Nacional de Ciencia y Tecnología (CONACYT) from the government of Mexico, and Consejo Estatal de Ciencia y Tecnología de Sonora (COECYT Sonora) for their financial support.

Thanks to Dr. Jean-François Joly for his guidance in the research project.

Special thanks to the Department of Mechanical and Aerospace Engineering and the Faculty of Graduate Studies and Research of Carleton University for the opportunity to pursue my masters degree. 
To my family

"Forget not Laila who re-walks the same path" 


\section{Table of Contents}

Abstract $\quad$ iii

Acknowledgments $\quad$ v

Table of Contents vii

List of Tables $\quad$ ix

List of Figures $\quad \mathrm{x}$

Nomenclature $\quad$ xiii

1 Introduction 1

2 Metallic Glasses and Atomistic Simulation 6

2.1 Metallic glasses . . . . . . . . . . . . . . . . . 9

2.1.1 Structure of metallic glasses . . . . . . . . . . . . . 12

2.1.2 Deformation of metallic glasses . . . . . . . . . . . . 15

2.2 Atomistic simulation . . . . . . . . . . . . . . . . 17

2.2.1 Interatomic potential . . . . . . . . . . . . . . 20

2.2 .2 Lattice structure . . . . . . . . . . . . . . . . . . . . 21

2.2.3 Energy minimization ................ 22

2.3 Atomistic simulation of metallic glasses . . . . . . . . . . . . . 23 
3 Wang Tiling $\quad 27$

3.1 Stochastic Wang tiling . . . . . . . . . . . . . . 28

3.2 Current state of the art of Wang tilings . . . . . . . . . . . . . 30

3.2.1 Wang tilings in engineering . . . . . . . . . . . . 31

3.2.2 Wang tilings in atomistic simulation . . . . . . . . . . 33

4 Methodology $\quad 36$

4.1 True specimen . . . . . . . . . . . . . . . . . . . 37

4.2 Wang tiling specimen . . . . . . . . . . . . . . . . . . . 43

4.2.1 Sample extraction . . . . . . . . . . . . . . 46

4.2.2 Prototile assembly . . . . . . . . . . . . . . . . 49

4.2.3 Wang tile construction ............... 54

4.2.4 Wang tiling assembly . . . . . . . . . . . . . . . 56

5 Results Analysis $\quad 59$

5.1 Macroscopic observations . . . . . . . . . . . . . . 60

5.2 Microscopic observations . . . . . . . . . . . . . 69

5.2.1 Atomic Bond Deficiency defects (BD defects) . . . . . . . . 70

5.2.2 Local atomic Mises strain, $\eta_{i}^{\text {Mises }}$, observation . . . . . . . . 77

5.3 Results analysis . . . . . . . . . . . . . . . . . 85

6 Conclusions $\quad 87$

6.1 Methodology findings . . . . . . . . . . . . . . . 88

6.1.1 True specimen findings . . . . . . . . . . . . . . 88

6.1.2 Wang tiling specimen findings . . . . . . . . . . . . . 89

6.2 Validation . . . . . . . . . . . . . . . . . . . 90

$\begin{array}{ll}\text { List of References } & 92\end{array}$ 


\section{List of Tables}

2.1 Generalized differences between crystalline and amorphous solids . . . 8

2.2 General attributes found in metallic glasses of different compositions (retrieved from $[1]) \ldots \ldots \ldots \ldots$

2.3 Comparison of tensile properties of various metallic glasses and stainless steel . . . . . . . . . . . . . . . . . .

4.1 Elastic constants for eight different specimens, where the Bulk and Young Modulus are measured in $\mathrm{eV} / \AA^{2}$. . . . . . . . . . . . . . . . . 42

4.2 Wang tile set composed of 16 distinct Wang tiles. Tiles are created by placing four different samples in a specific orientation (NSEW). . . 


\section{List of Figures}

1.1 Classification of solids. . . . . . . . . . . . . . . . . . 2

2.1 Representation of a unit cell in a three dimensional cubic lattice. . . . 7

2.2 Materials-selection chart. . . . . . . . . . . . . . . . . . 10

2.3 Comparison of the critical cooling rate and reduced glass transition temperature of glasses. . . . . . . . . . . . . . . . . . 11

2.4 Models of the amorphous structure. . . . . . . . . . . . . . . 14

2.5 Periodic boundary conditions. . . . . . . . . . . . . . . . . . . 21

2.6 Schematic representation of the potential energy landscape. . . . . . . 23

3.1 Wang tiling components. . . . . . . . . . . . . . . . 28

3.2 Stochastic Wang tiling components. . . . . . . . . . . . . . . . 29

3.3 Microstructure compression samples. . . . . . . . . . . . . . . . 32

3.4 Tiled microstructure compression sample. . . . . . . . . . . . . 32

4.1 True specimen. . . . . . . . . . . . . . . . . . . . . . 39

4.2 Specimen nucleation during minimization due to low initial density. . 41

4.3 Phase segregation of particles during minimization due to low initial

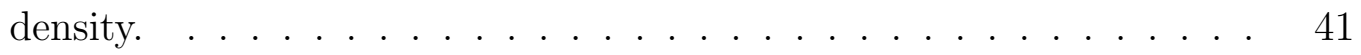

4.4 Graphic determination of the Young modulus. . . . . . . . . . . 43

4.5 Smaller true specimen. . . . . . . . . . . . . . . . . . . . . 44

4.6 Square samples extracted from a true specimen. . . . . . . . . . . . 47 
4.7 Modified Image Quilting Algorithm (IQA) $\ldots \ldots \ldots \ldots \ldots$

4.8 Prototile before and after minimization $\ldots \ldots \ldots \ldots$

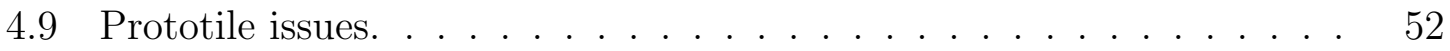

4.10 Wang tile representation inside a prototile. . . . . . . . . . 54

4.11 Wang tile extracted from a prototile. . . . . . . . . . . 54

4.12 Wang tile produced from a minimized prototile. . . . . . . . . 55

4.13 Wang tile assembly. . . . . . . . . . . . . . . . 56

4.14 Local atomic Mises strain, $\eta_{i}^{\text {Mises }}$, analysis of a Wang tiling specimen

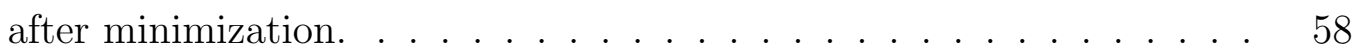

5.1 Specimen dimensions. . . . . . . . . . . . . . . . . . . . . . 61

5.2 Stress-strain curve comparison for eight different true specimens. . . . 62

5.3 Estimation of the elastic limit from the stress-strain curve. . . . . . 63

5.4 Stress-strain curve comparison for two different tilings (same tile set). 65

5.5 Stress-strain curve comparison for three different tilings (different sam-

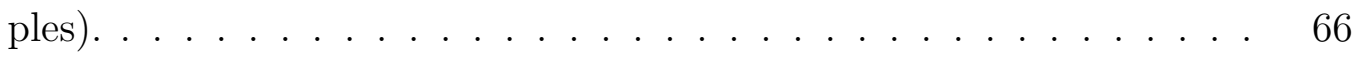

5.6 Stress-strain curve comparison for eight different tilings with samples from different specimens. . . . . . . . . . . . . . . . . 67

5.7 Stress-strain curve comparison for a true specimen and a Wang tiling

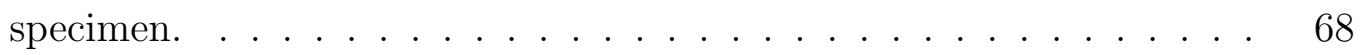

5.8 Vacancy defect and atomic Bond Deficiency (BD) defect comparison. 71

5.9 Example of a BD defect in a Wang tiling specimen . . . . . . 72

5.10 True specimen BD defects. . . . . . . . . . . . . . . . 74

5.11 Wang tiling specimen BD defects. . . . . . . . . . . . . 74

5.12 BD defect formation . . . . . . . . . . . . . . 75

$5.13 \mathrm{BD}$ defects at the maximum strength, $t_{\max } \ldots \ldots \ldots \ldots$

5.14 Timesteps of interest for visualizing the local atomic Mises strain, $\eta_{i}^{\text {Mises }} .79$ 
5.15 Shear Transformation Zone (STZ) before and after deformation. . . . 80

5.16 Intensity plots of $\eta_{i}^{\text {Mises }}$ for $t_{1}$. Atoms are coloured according to their

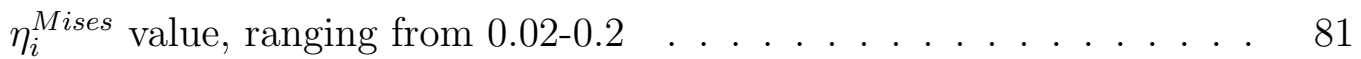

5.17 Intensity plots of $\eta_{i}^{\text {Mises }}$ for $\epsilon_{x x}=2 \%$. Atoms are coloured according to their $\eta_{i}^{\text {Mises }}$ value, ranging from $0.02-0.2 \ldots \ldots$. . . . . . . . 82

5.18 High $\eta_{i}^{\text {Mises }}$ value zone in the Wang tiling specimen. Atoms are coloured according to their $\eta_{i}^{\text {Mises }}$ value, ranging from 0.02 to 0.2 . . 83

5.19 Evolution of a zone with high values of $\eta_{i}^{\text {Mises }}$ from $t_{1}$ to $t_{\max }$ to $\epsilon_{x x}=$

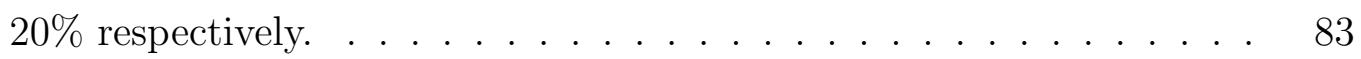

5.20 Intensity plots of $\eta_{i}^{\text {Mises }}$ for $t=t_{\max }$. Atoms are coloured according to their $\eta_{i}^{\text {Mises }}$ value, ranging from $0.02-0.2 \ldots \ldots$. . . . . . . . . 84

5.21 Intensity plots of $\eta_{i}^{\text {Mises }}$ for $\epsilon_{x x}=20 \%$. Atoms are coloured according to their $\eta_{i}^{\text {Mises }}$ value, ranging from $0.02-0.2 \ldots \ldots$. . . . . . . . . . 85 


\section{Nomenclature}

\begin{tabular}{ll}
\hline Acronym & Abbreviation for \\
\hline MD & Molecular Dynamics \\
MS & Molecular Statics \\
BMGs & Bulk Metallic Glasses \\
BD defects & Atomic Bond Deficiency defects \\
$\eta_{i}^{\text {Mises }}$ & Local atomic Mises strain \\
EAM potential & Embedded-Atom Model potential \\
IQA & Image Quilting Algorithm \\
LAMMPS & Large-scale Atomic/Molecular Massively Parallel Simulator \\
OVITO & Open VIsualization TOol \\
& \\
\hline
\end{tabular}




\section{Symbol Variable}

\begin{tabular}{|c|c|}
\hline $\mathrm{eV}$ & Electron-volt \\
\hline$\stackrel{\circ}{A}$ & Angstrom \\
\hline $\mathrm{T}_{r g}$ & Reduced glass transition temperature \\
\hline $\mathrm{T}_{g}$ & Glass transition temperature \\
\hline $\mathrm{T}_{m}$ & Melting temperature \\
\hline $\mathrm{T}_{c}$ & Crystallization onset temperature \\
\hline $\mathrm{E}$ & Young's modulus \\
\hline$\sigma_{f}$ & Fracture strength \\
\hline $\mathrm{E}$ & Total energy \\
\hline $\mathrm{F}_{i}$ & Embedding energy \\
\hline$\phi_{\alpha \beta}$ & Pair potential \\
\hline$r_{i j}$ & Interatomic distances \\
\hline$\rho$ & Density \\
\hline$\sigma_{x x}$ & Stress \\
\hline$\varepsilon, \epsilon$ & Strain \\
\hline$\dot{\varepsilon}$ & Engineering strain rate \\
\hline$L_{b o x}$ & Length of simulation box \\
\hline
\end{tabular}




$\begin{array}{ll}t & \text { Time } \\ \boldsymbol{d} & \text { Vector separation } \\ \boldsymbol{J} & \text { Jacobian } \\ \mathrm{N}_{i} & \text { Nearest neighbour atoms } \\ \mathrm{I} & \text { Identity matrix }\end{array}$




\section{Chapter 1}

\section{Introduction}

One of the greatest attributes of the human species is the ability to make things. The use of materials for specific purposes such as engineering, tools, components, etc have led to the technological world we live in.

The role of materials has been so important in history that historians and anthropologists have named time periods according to the most predominant material used, e.g. Stone, Bronze and the Iron Ages. Nowadays, we would not be able to identify a predominant material; humans have improved old materials and developed new materials to meet specific needs. A multitude of sophisticated materials are now available i.e. plastics, silicon, titanium, alloys, high-technology ceramics, composites, optical fibres, etc. It is due to the wide use of all these materials that we can refer to our modern society as the 'Age of Technology' [3].

The constant improvement on materials by artisans and technologists across history resulted in sophisticated products. Such improvement can be easily appreciated in our daily lives: the clothing we wear is adaptable to our surroundings. The so-

phistication of our weapons, starting from sticks and stones, to swords, to machine artillery and finally to chemical weaponry. Vehicles are no long pulled by horses, they can go longer distances and at a quicker pace than ever before. We no longer 

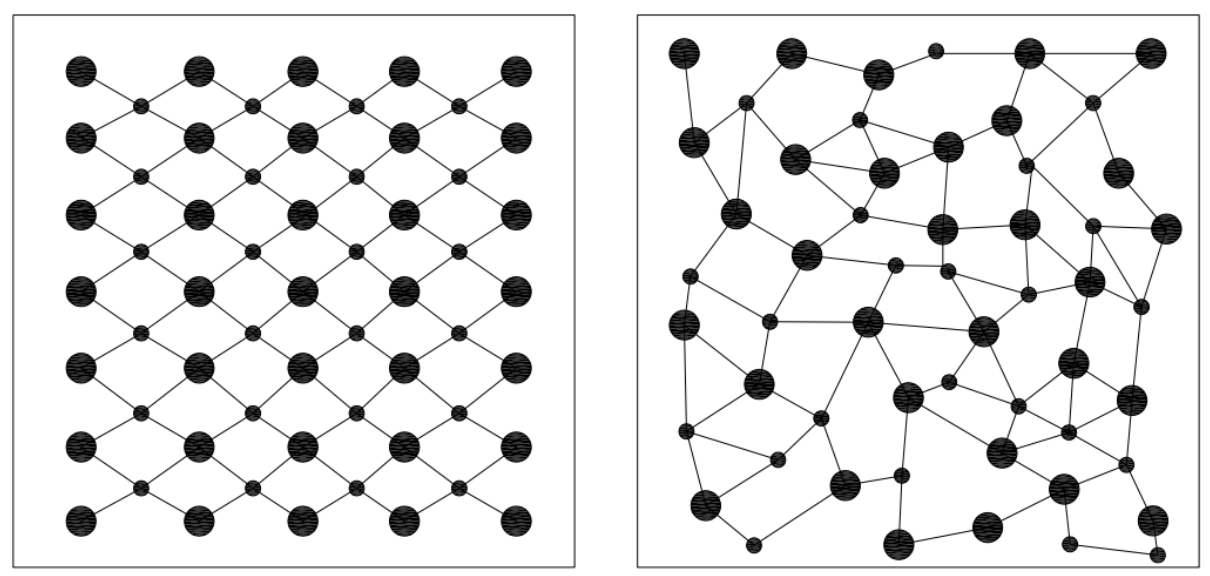

Figure 1.1: Classification of solids.

Crystalline solid (left) and amorphous solid (right).

live under sheds and preserve heat through natural fires; the comfort of our homes is possible thanks to the development of new materials that are less flammable and good heat conductors. Products have been refined with the adaptation of materials.

The adaptation of materials can be attributed to the evolution of science and our ever-growing knowledge of our surroundings. The more knowledge we possess of a material's physical and chemical properties the more implementations it can have. The atom is the basic unit of internal structure for studies in materials; while we know that all matter is made up of atoms, it is important to remember that not all atoms are the same. The atoms of a solid can be arranged following a repetitive three-dimensional structure, or, with no particular geometrical arrangement [4]. The first case, produces a crystalline solid and the second an amorphous solid, as seen on figure 1.1. A solid's durability, conductivity, and other properties, are directly related to it's atomic arrangement.

Centuries of research on crystalline solids have lead to the classification of crystal solids in accordance to their lattice structure, known as the Bravais lattices [5]. Each lattice is a unique combination of a crystal system and the centerings within the unit 
cell. This Bravais structure that a material presents, determines the solid's physical characteristics and engineering properties (i.e. conductivity, resistivity, stress, ductility, among others). Phase transitions and responses to mechanical or electrical prompts are direct manifestations of their crystallinity and defects.

Amorphous solids, on the other hand, cannot be classified to such an extent due to the lack of a definite geometrical order in their constituent particles. They are short range order solids that when broken produce fragments with irregular surfaces. When an amorphous solid, usually translucent or transparent, behaves like a solid, we call it a glass [6]. This research work is specific to a type of amorphous solid called a metallic glass.

Metallic glasses are usually alloys with a disordered atomic structure. They can form in several ways, i.e. extremely rapid cooling (also known as quenching) mechanical alloying, chemical reactions or radiation exposure [1].

The first reported metallic glass was documented in the early 1960s. We can therefore consider them a relatively modern material [1]. The novelty of metallic glasses points to a relative lack of information on their atomic structure and thermal and mechanical properties. Since metallic glasses are amorphous solids that do not follow a geometrical array, their representation is limited by the more-or-less random positions atoms occupy in the structure and limitations related to atom proximities. In order to better understand these interactions we perform atomistic simulations, such as Molecular Dynamics (MD).

MD is a computer simulation method that studies the displacement of atoms and molecules during a fixed period of time [7]. It studies the evolution of a system as a function of time due to interatomic (atom-to-atom) interactions. The accuracy in the method depends on the system's realism and the atomic interaction model 
used. Atomistic simulation is validated through experimental data and its use enables the study of structures at extreme conditions that are hard to attain through experimentation, and can be time consuming and expensive.

This research project arose from the complexity associated with the representation of metallic glasses in atomistic simulations. The majority of the amorphous systems studied in the literature have started from a randomly generated initial structure that then needs to be stabilized, or equilibrated. This endeavour can be time consuming and a truly equilibrated "groundstate", or local minimum, is difficult to achieve.

This thesis implements a mathematical model that has been successful in other fields (such as the graphics design field), known as Wang tilings [8]. It consists of the creation of samples (extracted from an amorphous specimen), that when assembled, produce a semi-random structure that looks non-repetitive to the human eye (termed 'Wang tiling specimen'). Few attempts have been made to implement this model in the study of amorphous solids, hence, a lot of concerns need to be addressed.

The accurate representation of metallic glasses, or other amorphous solids, via Wang tilings would permit the study of bigger systems than can then be studied using periodic MD simulations [1].

The main objectives of this thesis are to:

1. Adapt the Wang tiling model to atomistic simulations.

2. Develop a successful methodology for the creation and implementation of a Wang tiling metallic glass.

3. Verify the accuracy of a semi-random Wang tiling system in comparison to a random system.

In order to understand the relevance of the implementation of this model, it is important to highlight the characteristics of amorphous structures and the difficulty 
associated with their study. Chapter 2 provides an introduction to metallic glasses, how they are produced, their properties and their importance for engineering purposes. In chapter 2 the basics on atomistic simulations and the simulation of glasses is introduced. The goal of chapter 2 is to present different approaches on the study of metallic glasses and the limitations associated with them. It also reflects on the current state of the art of atomistic simulations for metallic glasses.

The description of the Wang tiling model is detailed in chapter 3. This chapter also reflects on the current state of the art of Wang tilings in engineering. It presents the most relevant studies being carried out and their contributions and implications for this thesis.

By the time the reader has read the first few chapters, they will have a better understanding of metallic glasses, Wang tilings and modeling glasses using atomistic simulations. The ensuing chapter, chapter 4, will present the methodology used to develop Wang tiling metallic glasses and the creation of a true specimen. The true specimen will then be used as the reference configuration for generating the Wang tiling specimen. Details on the limitations encountered during the methodology development will be discussed in chapter 5 and the comparison between a purely random (true specimen) and a semi-random (Wang tiling specimen) system will be addressed. Finally, the last chapter will present the conclusions to this thesis and future work. 


\section{Chapter 2}

\section{Metallic Glasses and Atomistic Simulation}

The introduction presented a broad classification of solid materials into crystalline or non-crystalline. The scope of this thesis is on non-crystalline solids, also known as amorphous materials, and specific to metallic glasses.

The introduction to this chapter expands on the differences between both categories (crystalline and amorphous) and sets the scene for discussing metallic glasses. Section 2.1 provides a brief historical recollection on the study of metallic glasses that provides insights on their limitations and unexplored potential.

Metallic glasses were discovered in the 1960's through experimentation and since then, research into their properties has been carried out both experimentally and with the use of computational resources. In section 2.2 atomistic simulation is introduced along with it's use for the study of amorphous solids. Section 2.3 reflects on the current state of the art of atomistic simulations of metallic glasses.

By the end of the chapter, the reader should have a general understanding on what a metallic glass is, how it differs from crystal solids, the impact of these differences on their properties (both physical and mechanical) and deformation, and a general understanding on atomistic simulations and their limitations when simulating metallic glasses. 
Solids can be classified as crystalline or amorphous depending on their atom arrangement. A solid is considered crystalline when it's atoms, ions, molecules or other constituent elements are arranged in a periodic pattern. We define their crystallinity as a repetition of a unit, known as a unit cell. A perfect crystal is that in which the atoms are arranged in a pattern that repeats periodically in three dimensions to an infinite extent [9], see figure 2.1 for a finite example.

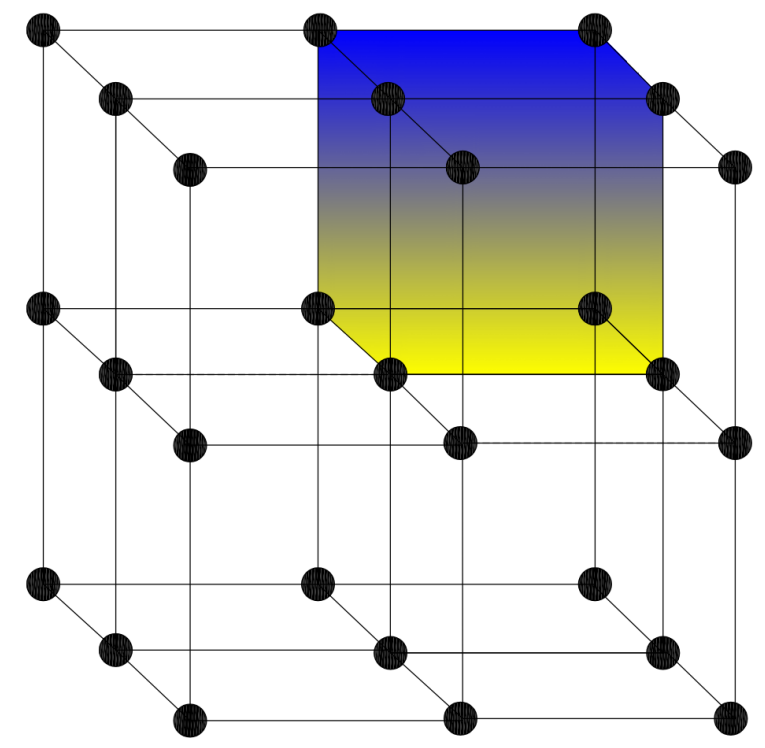

Figure 2.1: Representation of a unit cell in a three dimensional cubic lattice.

Amorphous solids are considered disordered structures that do not follow a set pattern during their creation. Disorder does not mean mere chaos, it refers to the breaking of symmetry and order. In the physics of these materials, we cannot rely the theorems or principles that are accepted for crystalline materials because their lattice translational invariance cannot be assumed.

In these disordered materials, two elementary features of a crystalline state may still be present: close-packed systems and homogeneousness [10]. Many disordered 
Table 2.1: Generalized differences between crystalline and amorphous solids

\begin{tabular}{|c|c|c|}
\hline Property & Crystalline Solid & Amorphous Solid \\
\hline Geometry & $\begin{array}{c}\text { Constituent particles are } \\
\text { orderly arranged in a repetitive } \\
\text { pattern. }\end{array}$ & $\begin{array}{r}\text { Particles do not follow a geometrical } \\
\text { arrangement. }\end{array}$ \\
\hline Melting Point & $\begin{array}{c}\text { Sharp melting point, the } \\
\text { solid changes state at a } \\
\text { defined temperature. }\end{array}$ & $\begin{array}{c}\text { Does not have a sharp melting point. } \\
\text { When an amorphous solid is heated, } \\
\text { it softens and starts flowing without } \\
\text { undergoing a sharp state change. }\end{array}$ \\
\hline Cooling Curve & $\begin{array}{c}\text { Cooling curve exhibits two breaks } \\
\text { that represent the beginning and the } \\
\text { end of the process of crystallization. }\end{array}$ & Smooth cooling curve. \\
\hline Cutting & Clean cleavage. & Irregular cut. \\
\hline
\end{tabular}

materials are as closed-packed as possible within their geometrical or chemical constraints. Close packing implies a high degree of uniformity in the density of the assembly, meaning that any small volume can be exchanged for a similar volume elsewhere without significant differences in properties; the system can therefore be defined as being homogeneous.

Because of their atomic arrangement, solids exhibit very different physical properties, some of these differences can be appreciated in table 2.1. One of the biggest differences between crystalline structures and amorphous materials is the great flexibility in the nearest-neighbour arrangement of atoms that the latter present; metallic glasses exhibit short-range order [1]. 


\subsection{Metallic glasses}

Metallic amorphous alloys, also known as metallic glasses, are newcomers to the amorphous materials group. Amorphous metals can be produced through a variety of ways, the most common being their production by quenching from a melt. They differ from ordinary structural metal in that their constituent atoms are not assembled in a crystalline lattice as was explained in the prior section. Amorphous alloys were first developed over 50 years ago and were used as magnetic core or reinforcement added to other materials. The formation of the first metallic glass is attributed to Duwez at Caltech, USA, in 1960 composed of $\mathrm{Au}_{75} \mathrm{Si}_{25}$ [11]. Their method permits large quantities of an alloy to be made into a glassy state or frozen liquid configuration by rapid quenching techniques at very high rates of $10^{5}-10^{6} \mathrm{~K} / \mathrm{s}$.

Research on metallic glasses gained momentum in the early $70 \mathrm{~s}$ and $80 \mathrm{~s}$. The novelty of these materials and their prospective applications lead to the creation and study of metallic glasses with a wide variety of chemical configurations. It took little time for the development of new 'materials-selection charts' that would compare the properties of newly developed metallic glasses to conventional engineering materials. These material selection charts permit a quantitative comparison of properties such as the elastic limit, Young Modulus, resilience, loss coefficient, etc. Figure 2.2 is an example of these charts, it was developed by Ashby et al. [12] as a resource for

the selection of materials based on properties of interest; in [12], several graphs are presented to compare the mechanical properties of metallic glasses. On charts of this type, the ellipses enclose the range of values associated with a specific group. A more detailed interpretation of figure 2.2 and the description of the properties of these glasses will be presented in section 2.1.1. From the figure, we can clearly determine that a greater strength is exhibited by metallic glasses in comparison to tungsten and 


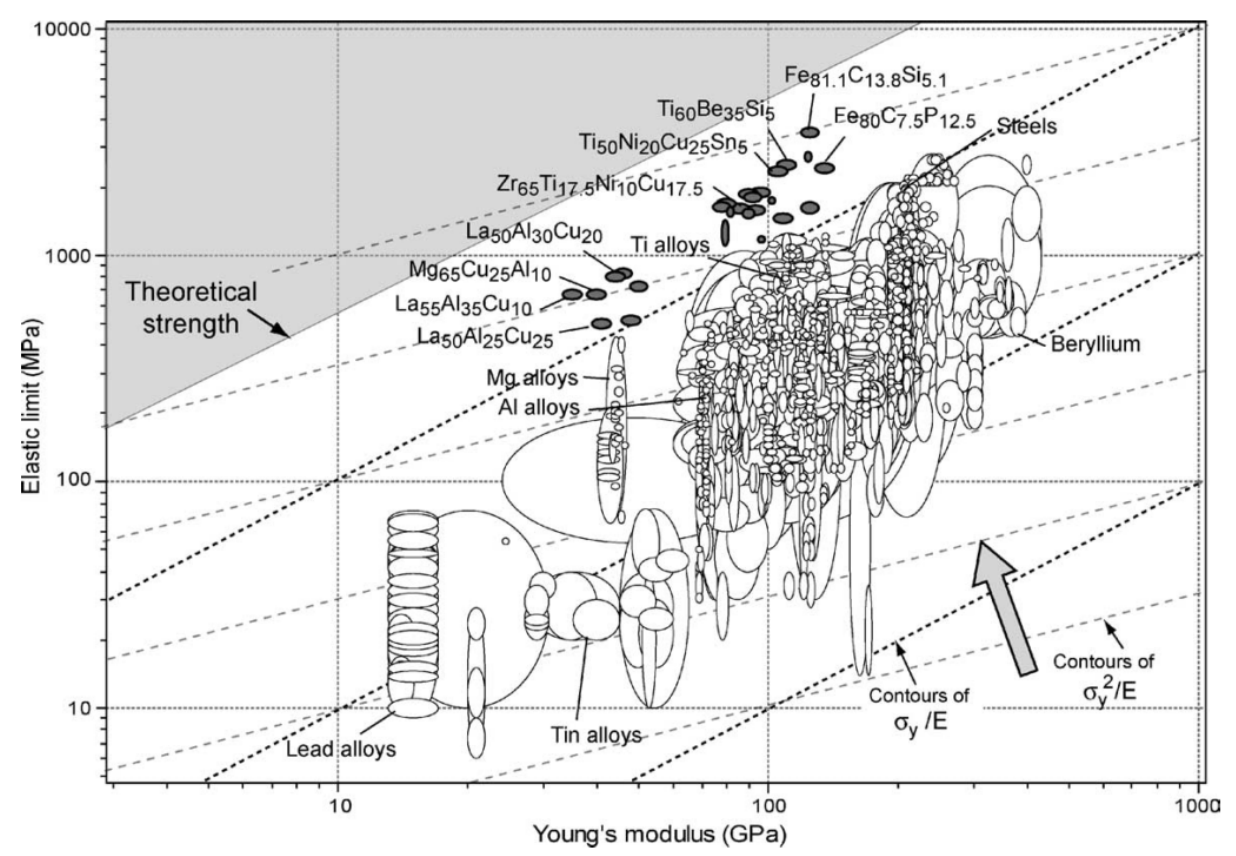

Figure 2.2: Materials-selection chart.

Elastic limit, $\sigma_{y}$, plotted against the Young's modulus, E. The graph includes information for 1507 materials grouped as metals, alloys, metal matrix composites and metallic glasses. Metallic glasses are located in the upper section of the image and are grouped according to their chemical compositions. Ellipses enclose the range of values associated with a specific group. Reproduced from [12].

steel alloys.

Amongst the many academics that have researched these materials, Turnbull and coworker made a crucial contribution to the discipline. They showed the similarities between metallic glasses and other glasses such as silicates, ceramic glasses and polymers. They showed that a glass transition manifested in conventional glass-forming melts also could be found in rapid quenched metallic glasses [13]. The glass transition occurred at a well-defined temperature, characteristic of crystalline solids, till then un-associated with amorphous solids. In his research, Turnbull established one of the criteria used for classifying glasses due to their glass-forming ability (GFA), known as the reduced glass transition temperature, $T_{r g}$. The $T_{r g}$ is the ratio between the glass 


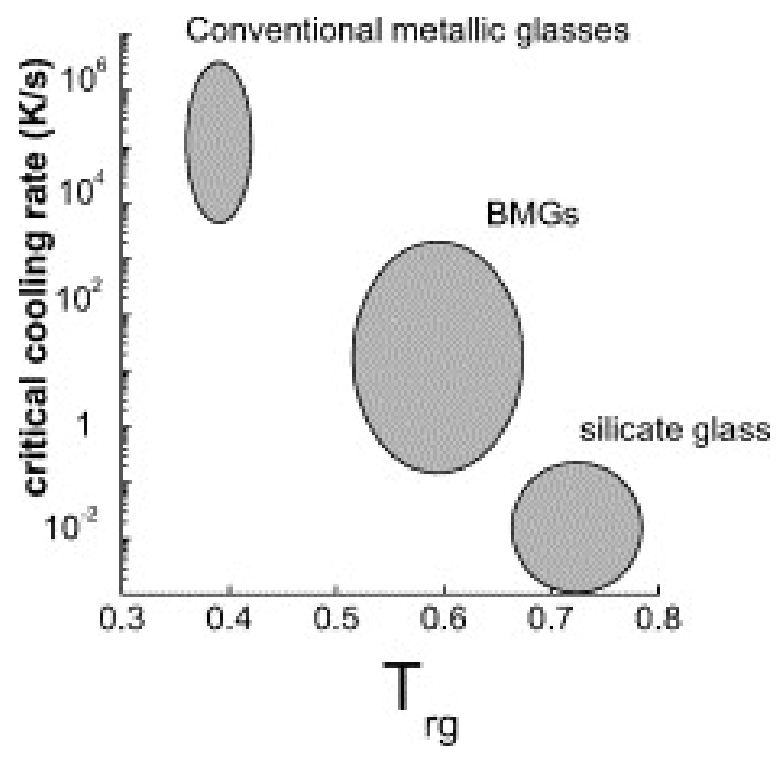

Figure 2.3: Comparison of the critical cooling rate and reduced glass transition temperature of glasses.

Includes information for three different material groups: conventional metallic glasses, bulk metallic glasses (BMGs), and sillicate glasses. Reproduced from [14].

transition temperature and the melting temperature, $T_{g}$ and $T_{m}$ respectively; where $T_{r g}=T_{g} / T_{m}$. The understanding of this criterion led to a more refined classification of glasses which can be appreciated in figure 2.3 [14].

Figure 2.3 shows a distinction between metallic glasses and bulk metallic glasses (BMGs). The classification is based on the size of the metallic glass formed during quenching. Glass that is larger than $1 \mathrm{~mm}^{3}$ is considered a BMG and a smaller sample is simply considered a metallic glass. Up until the 1980's only a limited number of BMGs were developed due to the size constraint. Nowadays there is a wide variety of BMGs that have been minutely classified by Takeuchi et al. according to: the atomic size difference, heat of mixing, and period of the constituent elements in the periodic table (reference [15] provides a diverse number of classification tables based on this criteria).

Early approaches to the fabrication of metallic glasses were mostly empirical and 
metallic glass production was considered accidental or due to production flaws. Turnbull's research provided the relationship between metallic glasses and other glasses and highlighted the performance dependence of a metallic glass to its glass transition rate, this discovery set the guidelines for the creation of new metallic glasses. Later on, researchers started to understand that not only were the quenching rate and temperature factors in the production of these glasses, but that the choice of constituent elements would also change the cooling rates and sizes of glasses generated. The accurate selection of constituent elements could result in cooling rates in the range of 1-100 K/s. Slower cooling rates meant that larger pieces could be fabricated, leading to higher applicability. For these alloys, the intrinsic factors, i.e. number of atoms, purities and the atomic size of the constituent elements, composition, cohesion among the elements, etc, played key roles in the glass formation [1]. The variety of attributes presented by BMG's has determined their possible applications, as can be seen from table 2.2 .

Amorphous materials exhibit both brittle and ductile behaviour that in some instances can be comparable to deformation in crystals at the macroscopic level [16]. Metallic glasses are new materials that exhibit very high strength, low internal friction and good corrosion resistance [17]. Their properties make them useful for the creation of: tools with sharp edges, springs and golf clubs (which benefit from their high elastic energy storage and low damping), new fashion items and information storage [6].

\subsubsection{Structure of metallic glasses}

Metallic glasses display some interesting properties that are very attractive for new commercial endeavours, as can be appreciated from table 2.1. So far, amorphous solids have been defined simply as having random structures; no information has 
Table 2.2: General attributes found in metallic glasses of different compositions (retrieved from [1])

\begin{tabular}{|c|c|c|}
\hline Attribute & Attractiveness & $\begin{array}{c}\text { Metallic Glass } \\
\text { Composition }\end{array}$ \\
\hline Mechanical & $\begin{array}{l}\text { - High hardness, good wear } \\
\text { and abrasion resistance } \\
\text { - High yield strength } \\
\text { - High fracture toughness } \\
\text { - High specific strength } \\
\text { - Low mechanical damping }\end{array}$ & $\begin{array}{c}\mathrm{Fe}, \mathrm{Al}, \mathrm{Ti} \text { based } \\
\text { alloys }\end{array}$ \\
\hline Thermal & $\begin{array}{c}\text { - Supercooled liquid } \\
\text { when } \mathrm{T}_{g}<\mathrm{T}_{c} \\
\text { - Crystallization onset } \\
\text { temperature, } \mathrm{T}_{c}\end{array}$ & $\begin{array}{l}\mathrm{Pd}, \mathrm{Cu}, \mathrm{Si}, \mathrm{Ag} \\
\text { i.e. } \mathrm{Pd}-\mathrm{Cu}-\mathrm{Si} \& \\
\mathrm{Pd}-\mathrm{Ag}-\mathrm{Si} \text { alloys }\end{array}$ \\
\hline $\begin{array}{c}\text { Electric and } \\
\text { magnetic }\end{array}$ & $\begin{array}{c}\text { - High magnetic permeability } \\
\text { - Resistivity independent } \\
\text { of temperature }\end{array}$ & $\begin{array}{c}\mathrm{Fe}, \mathrm{Co}, \mathrm{Ni} \text { based } \\
\text { alloys }\end{array}$ \\
\hline Chemical & - Corrosion resistance & $\begin{array}{c}\text { B, Si, P based } \\
\text { alloys }\end{array}$ \\
\hline
\end{tabular}



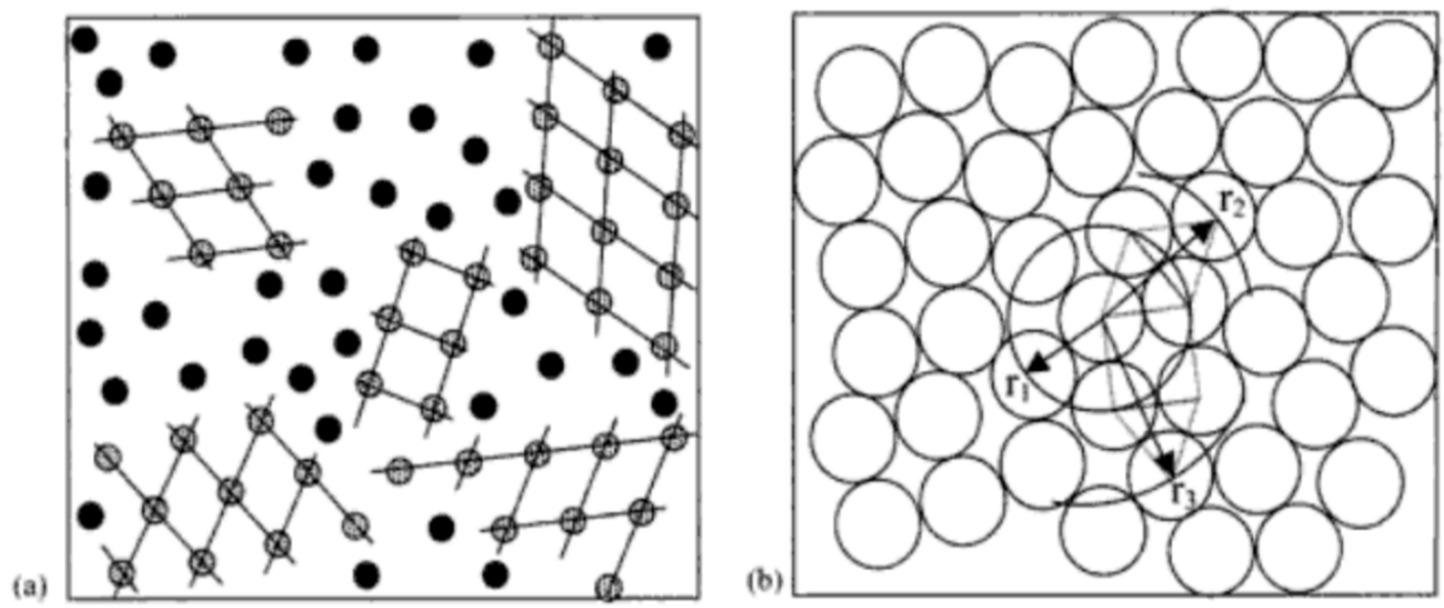

Figure 2.4: Models of the amorphous structure.

Microcrystalline disorder material (a), and topological disorder model (b). Reproduced from [18].

been provided on how we study this lack of geometrical order. There are two wellknown models for the representation of disordered structures: the microcrystalline disorder model and the topological disorder model, see figure 2.4. For the representation of metallic glasses, the microcrystalline disorder model is frequently used. This model reproduces the basic profile of the pair distribution function, or interatomic interactions, observed experimentally.

Figure 2.2 provides a more detailed resource for the comparison of metallic glasses to other materials. In the image, metallic glasses are identified by their composition (in at.\%), they stand out from other materials as they lie in the upper edge of the populated region. They have high strength in correlation with their Young's modulus, making them ideal for precision tools such as knives and scalpels. Also, their lack of grain structures allows for a closer edge sharpening than with crystals [6].

The tensile properties of metallic glasses can be seen in table 2.3. It clearly shows that metallic glasses have very high values of hardness and strength when compared to conventional materials such as stainless steel. Further interpretation of the table, such 
Table 2.3: Comparison of tensile properties of various metallic glasses and stainless steel

\begin{tabular}{|c|c|c|c|}
\hline Alloy system & $\begin{array}{c}\text { Fracture strength } \\
\sigma_{f}[\mathrm{MPa}]\end{array}$ & $\begin{array}{c}\text { Yield strength } \\
{[\mathrm{MPa}]}\end{array}$ & $\begin{array}{c}\text { Young modulus } \\
\mathbf{E}[\mathbf{G P a}]\end{array}$ \\
\hline $\mathrm{Cu}_{60} \mathrm{Hf}_{25} \mathrm{Ti}_{15}$ & 2010 & 2160 & 124 \\
\hline $\mathrm{Zr}_{70} \mathrm{Al}_{10} \mathrm{Ni}_{20}$ & 1411 & 1335 & 61 \\
\hline $\mathrm{La}_{55} \mathrm{Al}_{25} \mathrm{Ni}_{20}$ & 735 & 515 & 34 \\
\hline Stainless steel 304 & 505 & 215 & $193-200$ \\
\hline
\end{tabular}

as in the determination of the ratio of the fracture strength to the Young's modulus, is used for assessing high-strength materials. This particular value is considerably greater in metallic glasses, 0.03, compared to commercial alloys, 0.005 .

\subsubsection{Deformation of metallic glasses}

The use of metallic glasses in structural applications requires understanding their mechanical properties, especially the yield criteria.

Studying deformation in metallic glasses through atomistic simulation, specifically molecular dynamics, is a three step process:(i) form a random system and equilibrate it, (ii) quench the system to form a glass and (iii) apply a deformation to it. For the deformation step, one must first choose the type of deformation to perform on the system, i.e. shear or tension.

In the previous section, several chemical and mechanical properties of metallic glasses were presented. They are strong materials that exhibit a high elastic limit and fracture well beyond their yield point.

Deformation is different at high and low temperatures. When the temperature is smaller than the crystallization onset temperature, $T_{c}$, thin shear bands start to occur. When the temperature nears $T_{c}$, inelastic deformation starts to be present. In this 
stage, deformation is dependent on the strain rate; at high strain rates (approximately $10^{-3} / \mathrm{s}$ ) fracture takes place, while at low strain rates (approximately $10^{-5} / \mathrm{s}$ ) necking occurs and deformation is more homogenous [19].

One of the first atomistic descriptions of plastic deformation in metallic glasses is Argon's model of free volume [20]. This model describes localized regions of increased disorder. It considers that in some regions there is a lower coordination between atoms than in the rest of the material, known as free volume sites; in these regions, it is energetically more likely that localized shear transformations will occur. In his model, Argon postulated that at small temperatures and large applied stresses, many free volume sites will rearrange, and the localized sites would produce a new structure. Argon's model presented some problematic consequences as it implied a pressure sensitivity to deformation.

In 2008, Keryvin et al. [21] proposed the use of the Mohr-Coulomb (M-C) model which incorporates the effect of normal stresses on plastic deformation. This model was not developed specifically for the simulation of glasses, but for granular solids. However, metallic glasses can be considered analogous to granular solids as their motion of atoms is similar to the sliding of granules. This model is considered the most accurate for the representation of inelastic deformation; it confirms the influence of a normal stress component on the yielding criteria of metallic glasses.

Further research by Schuh and Lund [22] validated the M-C model for yield criteria. Their research rested on the model of fundamental units of deformation, defined as shear transformation zones, STZ's, regions with disordered atoms that reorganized themselves under stress.

Metallic glasses have a low ductility due to the localization of plastic deformation in thin shear bands. In these regions, local heating leads to decohesion and catastrophic failure of the glass [23]. 
Metallic glasses exhibit reversible elastic deformation at small applied stresses, irreversible deformation at large stresses and a strong dependence on the state of the system during past deformations [24]. This behaviour arises during shearing because first-nearest-neighbour relations and chemical ordering are severely disrupted at the atomic level [25]. Metallic glasses do not present long-range atomic order like crystalline materials do; long-range order leads to stability and durability. They have pronounced short- and medium- range order at the atomic scale making them less stable than crystals [26].

\subsection{Atomistic simulation}

The modern world is in constant demand of highly optimized efficient products. The production of these products is limited by our understanding of the relation between a material's microstructure and its response at the macroscopic level. Computational mechanics and atomistic simulations are two major resources that try to shed a light on this relation.

Computational modeling is a valuable asset that complements experimental research and helps validate new models. Simulations provide key tests of theory, providing insights that lead to new theoretical ideas and provide a bridge between theory and experiment. An advantage of atomistic simulations is the detailed picture of the model under investigation that they provide. They help explain the connection of macroscopic properties to the microscopic and even atomic scale.

Atomistic simulations require the use of a potential energy function that defines the interactions between atoms $[27,28]$. The positions and trajectories of atoms are determined through a system of coupled equations of motions, which result in a time evolution description of a system. With these quantities and using statistical 
mechanics and thermodynamics, various thermodynamic parameters and correlation factors can be derived $[27,29]$. The potential energy functions used for the description of metallic glasses in particular will be discussed later in section 2.2.1.

When modeling materials at the atomic scale resolution, methods like Molecular Dynamics (MD), Molecular Statics (MS) and Monte Carlo based simulations are standard methods. These simulations generate temporal-spatial information on twodimensional or three-dimensional atom configurations and their atom trajectories, which are then analyzed to better understand a physical system.

MD originated with the work of Alder and Wainwright [7] on the interaction of hard spheres and their thermodynamic interpretation. Since the 1960's, more sophisticated methods have been developed and refined with applications to liquids, solids and gases. MD is now one of the most popular tools for the exploration of molecular structures, thermodynamic properties and dynamics.

When working with isolated molecules, MD is about developing quantitative predictions of molecular size and shape, atom interactions, behaviour under pressure and the transition of molecules from one state to another. For bulk systems, the focus may be on thermodynamic properties or deformation response to loads. MD relies on time-steps that are used for sampling a probability distribution to describe the likely change a molecule might experience [30].

Classical mechanical models rely on the use of Newton's equations for motion of the nuclei. However, the application of these laws leads to equations much too complicated to be solvable analytically [31]. Applying Newton's Second Law to modeling materials, leads to equation 2.1, where the forces are determined from the potential energy function $U$. In equation $2.1, m$ is mass, $\boldsymbol{a}$ is the acceleration, $\boldsymbol{f}$ is force, and $\boldsymbol{q}$ is the position of the atom. 


$$
m \boldsymbol{a}=\boldsymbol{f} \quad \text { where } \quad \boldsymbol{f}=\frac{-\delta U}{\delta \boldsymbol{q}}
$$

Equation 2.1 must be supplemented by the initial conditions of all atoms (positions and velocities) for it to describe motion, it cannot be used by itself. This equation is solved discretely, and can be solved using the velocity-Verlet algorithm [32].

In this research, MD and MS calculations are conducted using a free, open source program called LAMMPS ${ }^{1}$ from Sandia National Laboratories [33].

Along with the accuracy of the model being used during an atomistic simulation, visualization software plays a key role in the translation of raw atomic coordinates into meaningful graphical representations that accurately depict the derived data. The visualization software used in this work is called OVITO ${ }^{2}[34]$. It covers both the analysis and the visualization of large-scale atomistic databases that are produced by Monte Carlo or Molecular Dynamic/Statics simulation codes. For this thesis, OVITO was used (i) as a visual aid to determine if systems were visually representative of amorphous structures (ensuring systems weren’t granulating), (ii) to visualize atom movement and system deformation in a time scale, (iii) to calculate the displacement between two states of the system, and (iv) for colour shading atoms for the visualization of atomic structures. Images from OVITO can be seen in chapters 4 and 5.

The next subsections aim to provide the reader with the necessary background for setting up an atomistic simulation and the implications associated with choosing a pair potential, a periodic lattice and an energy minimization algorithm. This information will be relevant for understanding the choices made when conducting this research, the specifics will be provided in chapter 4 .

\footnotetext{
${ }^{1}$ Acronym for Large-scale Atomic/Massively Parallel Simulator.

${ }^{2}$ Acronym for Open Visualization Tool.
} 


\subsubsection{Interatomic potential}

Potential models are constructed from special functional forms that describe interactions of pairs, triples or quadruples of atomic nuclei taking into account interactions predicted through quantum mechanics or observed in experiments. Simulations of liquid and glasses through potential models date back to the earliest stages of simulation studies. They have been used to answer basic questions regarding the properties of glasses and the nature of the glass transition. Several models have been used, including but not limited to: pair potentials, soft spheres, hard spheres, many-body interactions and $a b$ initio calculations.

When working with metallic glasses, many-body potentials are the most common empirical model used, therefore, a more detailed explanation is provided.

\section{Many-body potentials}

Many studies on glass formation are carried out using pairwise interaction potentials such as hard spheres, repulsive soft spheres, and the Lennard-Jones model. Recently, many-body interaction potentials are being used to simulate more realistic systems. Many-body interactions describe the energy of an atom as a function of a fictitious "electron density" from the neighbouring atoms, plus a pair term. These methods use equation (2.2) for the total energy, E, calculation, where $F_{i}$ is the embedding energy experienced by atom $i$ due to the sum of electron densities $\rho$ from all other atoms, $\phi_{\alpha \beta}$ is a pair potential and $r_{i j}$ are interatomic distances:

$$
E=\sum_{i} F\left(\overline{\rho_{i}}\right)+(1 / 2) \sum_{j \neq i} \phi\left(r_{i j}\right)
$$

Assuming that the density is spherically symmetric, the density is written as equation (2.3), where atoms within a specified cutoff radius are taken into account. 


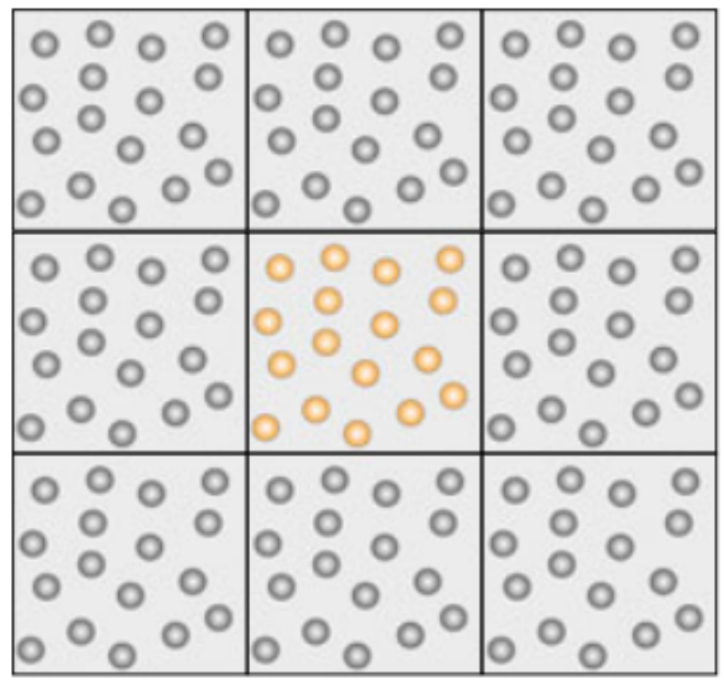

Figure 2.5: Periodic boundary conditions.

Representation of periodic boundary conditions for a two-dimensional system of interest (coloured orange in the image). Reproduced from [30].

$$
\overline{\rho_{i}}=\sum_{j \neq i} \rho_{j}\left(r_{i j}\right)
$$

In this research, the interaction between the molecules is determined by the embedded-atom model potential (EAM potential) for a copper, $\mathrm{Cu}$, and zirconium, Zr, system that was developed by Mendelev et al. [35]. The cutoff radius used in this work is the one reported in the EAM potential.

\subsubsection{Lattice structure}

The technique of periodic boundary conditions can be used in two-dimensional and three-dimensional systems. It assumes that a simulation box is surrounded by a box of particles whose positions are exact shifted images of those in the original system. When atoms move outside the box, they re-enter the box at a corresponding point in the opposite face, as seen in figure 2.5. 
This type of boundary condition is imporant in simulations of realistic systems where one wants to eliminate boundary effects. Periodic boundaries allow the preservation of Newton's third law on translational symmetry and thus the conservation of momentum. However, they impose a periodicity on the system that is unavoidable, even if one wants to study amorphous materials.

\subsubsection{Energy minimization}

As seen in section 2.2.1, we can compute the potential energy of a system of atoms based on interatomic distances between atoms. Given the potential energy function of all atoms in a system, it is possible to represent it in a potential energy landscape [32]. This landscape presents a $3 \mathrm{~N}$ dimensional (for $\mathrm{N}$ atoms) topology analogous to a terrestrial landscape, as can be seen in figure 2.6.

When seeking the most energetically stable configurations and their atomic arrangements, minima and basins are the most physically relevant features of the potential energy. The curvature of the energy function around a minimum determines things such as the elastic moduli of a solid.

It is of no surprise that methods to search for minima receive a lot of attention. The energy minimization of a system is performed iteratively by adjusting atom coordinates. Iterations are performed till a configuration has reached its local potential energy minimum; when this happens, we consider that the system is equilibrated.

In order to determine in which direction each atom should move, a minimization algorithm is used. This algorithm, sets the search direction along which atom coordinates are changed. This search normally evaluates forces and energies several times to determine the new coordinates. There are several minimization algorithms in existence, differing in complexity and the variables accounted for. In this work, two 


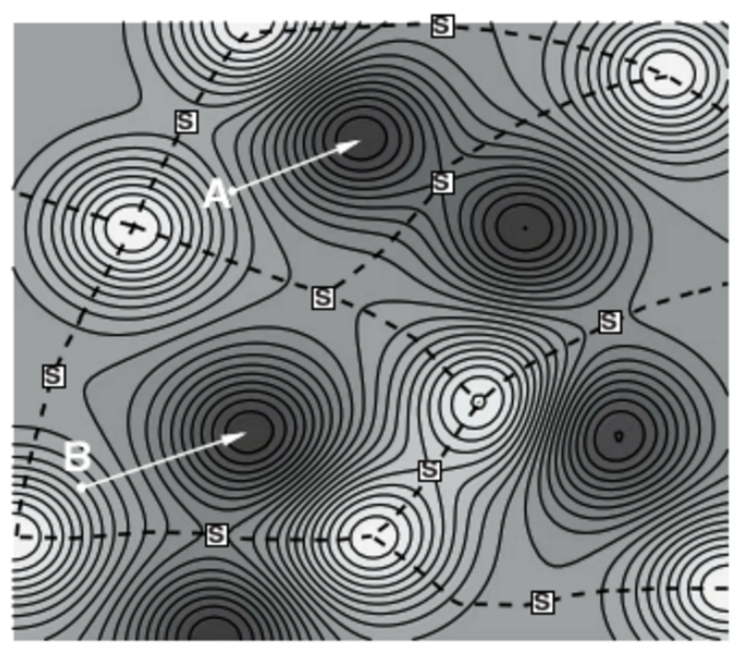

Figure 2.6: Schematic representation of the potential energy landscape.

The darkest regions are energy minima, lightest are energy maxima, and saddle points are indicated by "s". Solid lines are contours of constant energy and dashed lines are dividing surfaces that separate the different minima. Reproduced from [32].

algorithms were used: the Conjugate Gradient (CG) method and a damped dynamics method, known as 'FIRE'3 [36,37].

\subsection{Atomistic simulation of metallic glasses}

In section 2.2, a general background on atomistic simulations and it's implementation for the study of materials was presented. Simulations provide a detailed picture of a model and explain the relationship of macroscopic properties to the atomic scale. They can and are used to compare experimental results to a hypothetical model and validate it. This model can then be used to generate simulation results that would otherwise be inaccessible through experimentation. However, simulations also face a number of limitations.

Results of atomistic simulations are dependent on the interaction potential used.

\footnotetext{
${ }^{3}$ Acronym for Fast Inertial Relaxation Engine.
} 
In section 2.2.1 a brief description on interatomic potentials was provided. Typically, metallic glasses consist of two or more components [1] and developing an accurate potential, representative of experimental results, is not a trivial task [35].

Other limitations associated with the atomistic simulation of metallic glasses are related to: the availability of a phase diagram for a particular system, timescale dependencies and glass formation [1]. Being aware of these limitations ensures that the created system is representative of metallic glasses. This realism will be specially important for the creation of the initial configuration; the configuration from which a Wang tiling specimen will be formed.

The phase diagram provides information on the degree of undercooling and the driving force for crystallization [38]. If information on the phase diagram is unknown, simulations of binary or higher-order systems simply ignore it or use alternative techniques to calculate it. The phase diagram's information can be of value for establishing cooling and heating rates and ensuring glass formation.

In Molecular Dynamic (MD) simulations, interatomic interactions within a system are determined at each timestep (one femtosecond). The size of the system modelled has some influence in simulation results [1]. Present computational power allow the simulation of several thousand to a few million atoms, where the complexity of the system determines the size. Larger crystalline systems can be studied when compared to amorphous systems; this difference is due to the behaviour of amorphous systems, it is not as easy to predict as with crystalline systems and more complex algorithms are required for modelling them.

Another limitation, regarding the glass-forming ability, is of particular interest for metallic glasses. It has been shown that in quenching, the cooling rate has a direct effect on whether a system crystallizes or not [39]. Another factor limiting glass formation, is the system's density. The wrong density can result in phase segregation 
or nucleation of the components.

This research consists on the implementation of Wang tilings for the representation of metallic glasses. Broadly speaking, a Wang tiling is formed by arranging small components, Wang tiles, in a sequence until they tile a finite plane (specifics on Wang tilings can be found in chapter 3). The small components are taken from a reference configuration that is considered to be a metallic glass. Hence, it can be assumed that the accuracy in the representation of metallic glasses using Wang tilings is influenced by the reference configuration used (known as true specimen in this work). Therefore, it is important to remember the aforementioned limitations and ensure they are accounted for in the creation of the true specimen.

Ultimately, interest in metallic glasses resides in their mechanical properties. Atomistic simulations provide information which connect local structures with constitutive behaviours such as strength and strain softening. Systems can be subjected to different types of deformation, such as simple shear or tension, to determine whether shear banding or necking occurs [17].

Strain simulations aren't straightforward. In simple strain, at low temperatures, a small strain results in a proportional stress elastic deformation; and under constant strain a steady state Newtonian flow can be achieved; however, this is not always the case. Kob and Anderson [40] demonstrated that systems with a different initial temperature might produce either localized flow or homogenous flow (Newtonian flow) regardless of being quenched to the same temperature and deformed under identical conditions. Even for identical initial structures and temperatures, the history of the system is important. Rattler and Robbins [41] performed simulations that showed that the shear yield stress increases logarithmically with time.

If the deformation results in shear band formation, recent work indicates that shear banding changes the structure in the glass and that this softening can be reversed by 
annealing the sample. However, Albano and Falk [40] showed the difficulty in making definitive statements as identical compositions could present different deformation under identical simulation conditions.

The system's mechanical response to deformation can be used for determining the differences between systems. This will be used to compare the Wang tiling specimen to the true specimen (reference configuration) to assess the ability of the Wang tiling specimen to reproduce the true response.

Atomistic simulation has been used for the study of amorphous solids and metallic glasses in particular [1]. The basic characteristic of a metallic glass is the lack of geometrical order, which results in a system that is difficult to model. The interatomic potential, system size, and timestep dependency of the system are some of the factors that need to be accounted for to ensure the creation of a metallic glass. Since a Wang tiling system is formed from a reference, a possible hypothesis is that the accuracy with which a Wang tiling specimen represents metallic glasses depends on the reference configuration used; if the reference is a good representation of metallic glasses, the Wang tiling system created will be a good representation as well.

One of the greatest limitations for studying metallic glasses is computational resources. Simulating large amorphous systems is time consuming and depends on the complexity of the modelled system. Implementing Wang tilings for the representation of metallic glasses might be a solution to this problem. A hypothesis is that a specimen created through Wang tiles (referred to as the Wang tiling specimen in this research) would take less time to minimize. This idea is based on the fact that a Wang tiling specimen is formed from a reference configuration (true specimen) that is already representative of metallic glasses. 


\section{Chapter 3}

\section{Wang Tiling}

In chapter 2, a description on metallic glasses and Molecular Statics (MS) and Dynamics (MD) was introduced. The objective of this thesis is the implementation of Wang tiling theory for the representation of metallic glasses in MD simulations. In this chapter, the history of Wang tilings and their application in different fields is presented. The final section, section 3.2, provides the current state of the art of Wang tilings and the importance of their implementation in atomistic simulations for the representation of amorphous solids.

In 1961, Hao Wang, American mathematician and logician presented the Wang tiling method [8], a geometrically motivated method based on the creation of square tiles with coded edges permitting their assembly in a geometrically compatible manner. His proposal included a semi-decisional procedure for the tile arrangement based on boundary compatibilities, as seen in figure 3.1. The resulting tiled plane was termed a Wang tiling (visually analogous to a chessboard).

Wang's work was based on the periodic arrangement of tiles for the creation of an infinite tiling, regardless of the number of tiles required for the endeavor. Following his work, researchers focused on finding a finite set of tiles that could tile the infinite 

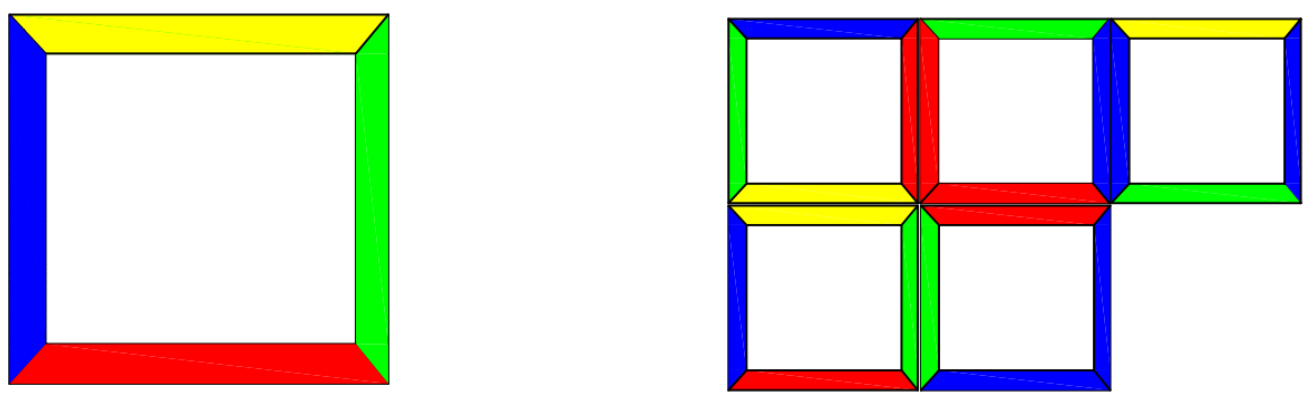

Figure 3.1: Wang tiling components.

Wang tile square with coded edges (left) and Wang tile arrangement (right), the resulting arrangement is known as the Wang tiling. Tiles are assembled ensuring edge code compatibility: interacting tiles present the same coloured edges.

plane aperiodically. Half a decade later, Culik [42] reduced the number to 13 tiles, which he then extended to include a three dimensional model of Wang cubes [43].

\subsection{Stochastic Wang tiling}

A tiling is formed by assembling small components (known as tiles). Using the tiling concept for modelling amorphous solids seems appealing, as large amorphous systems can be created using small amorphous components. However, each type of tiling requires a specific assembly procedure, a defined number of tiles, and edge constraints. Cohen et al. [44] introduced a stochastic variant to alleviate these restrictions. This stochastic variant allows for freedom in the choice of number of tiles and codes on tile edges.

The stochastic tiling starts with a Wang tile with coded edges (square domain with codes attributed to its edges), see figure 3.2 with colour coded edges. All types of tiles (such as the ones in the figure) that are available for assembly are known as the 'Wang tile set'. A tile that is rotated by multiples of $\pi / 2$ is considered a new tile type. The plane that is covered by the tiles is denoted as a tiling. 


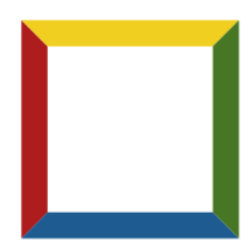

(a)

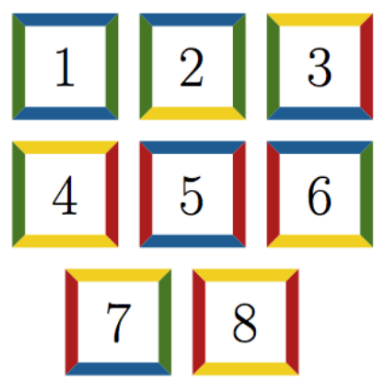

(b)

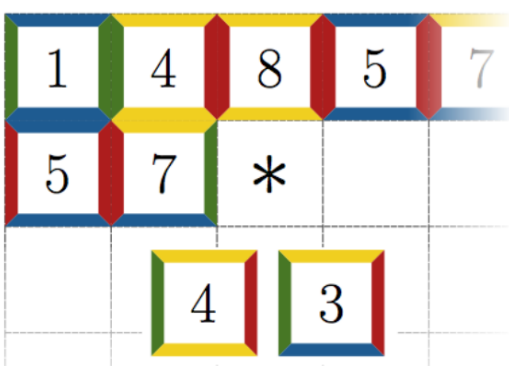

(c)

Figure 3.2: Stochastic Wang tiling components.

Wang tile (a), tile set (b), and tiling (c). The tiling assembly, image (c), shows two different compatible Wang tiles for the next location. Reproduced from [45].

The idea of a stochastic tiling is to cover an infinite plane with tiles; where adjacent tiles have the same edge code on touching edges, as seen in figure 3.2. Image (c) in the figure shows that two possible tiles can be assembled in the starred location (tiles 4 and 3 from the set in (b)); they both present a west-green and north-yellow boundary that is required. The tile that is placed is randomly chosen ensuring aperiodicity.

We can identify the basic elements of a Wang stochastic tiling as (i) Wang tiles, (ii) tile sets and (iii) tilings. Where:

1. Wang tile: square piece that carries micro-structural patterns within its domain. Edges are usually distinguished by colours, alphabetical codes or integers (figure $3.2(\mathrm{a}))$.

2. Tile set: collection of tiles that enable to cover an infinite plane aperiodically (figure $3.2(\mathrm{~b})$ ).

3. Tiling: discrete mapping of the tiles in a planar lattice (figure $3.2(\mathrm{c})$ ).

These definitions, are the basis for understanding the terminology used in chapters 4 and 5 . 
The steps for generating a stochastic Wang tiling filled up in a row-by-row order are: (i) the edge codes for the previous placed tiles are obtained (ii) tiles with the same edge code are identified from the tile set (iii) a tile from the subset is randomly selected (iv) the selected tile is placed in the tiling, see figure 3.2. The restrictions associated with creating a tiling are directly related to the assembly procedure. In order to construct a tiling, at least one tile for all admissible combinations on its edges needs to be available in the tile set. In order to ensure randomness, at least two tiles for each combination need to exist. The aperiodicity of the resulting tiling is guaranteed assuming the random number generator never returns a periodic sequence of numbers.

\subsection{Current state of the art of Wang tilings}

Little research has been done on the implementation of Wang tiling in atomistic simulations. An accurate representation of amorphous systems through Wang tilings can potentially enable researchers the study of larger systems created from a more stable initial structure; where the system would not need to be randomly generated from scratch. Also, one might be able to study a small set of tiles instead of a large random system.

There is great value in knowing the current state of the art of Wang tilings in engineering and the merits in its application in atomistic simulations of amorphous solids. This section provides information on both topics and highlights the value in implementing Wang tilings for the simulation of metallic glasses. 


\subsubsection{Wang tilings in engineering}

At first, Wang tiles were attractive exclusively to researchers in the area of Discrete Mathematics and the Theory of Computation. Their area of application changed in the mid 1980's, when Shechtman et al. [46] reported the first observation of a metallic crystal with icosahedral symmetry, exhibiting five-fold symmetry. That same year, Levine and Steinhard [47] achieved the same orientation with the Penrose kite and dart tiling [48] and termed solids without periodic translational order as 'quasicrystals'. The connection between quasicrystals and tilings then led to research on the thermodynamics of a tiling, particularly a set of 16 tiles called the Ammann tiles $[49,50]$. These were the first applications of tilings to the realm of Materials Science and Engineering.

Wang tiling applications are not limited to those three areas, the use of these tiles has also expanded to Biology. It has been used as a concept of matching rules in the design of DNA structures [51]. End sequences of DNA bipolymer strands are used to achieve edge compatibility. This application might later on be extrapolated for the assembly of nano-structures such as nano-wires [52].

Since the beginning of the century Wang tilings have been used in computer graphics for the production of irregular patterns. Cohen et al. [44] developed a way of designing the tiles from a provided sample and then made use of an image merging algorithm developed by Efros and Freeman for the assembly of the image-tiles [54]. This approach was then adapted to Materials Engineering by Novák, to reconstruct disordered microstructures [53] and amorphous solids [55], figure 3.3. Novák studied the compression of random microstructures that were produced via stochastic Wang tilings, his work is the first to implement Wang tilings for the representation of disordered microstructures. This research is an extension of part of the work developed 


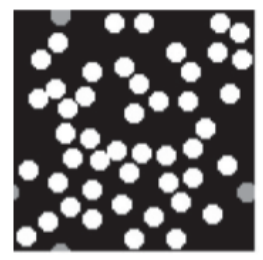

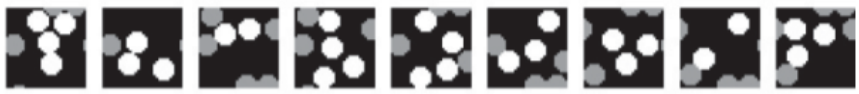

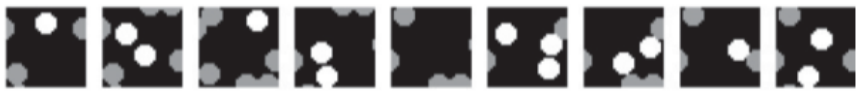

Figure 3.3: Microstructure compression samples.

Representation of a single cell containing the complete morphological information (left) and the building blocks assembled to produce a tiling (right). Reproduced from [53].
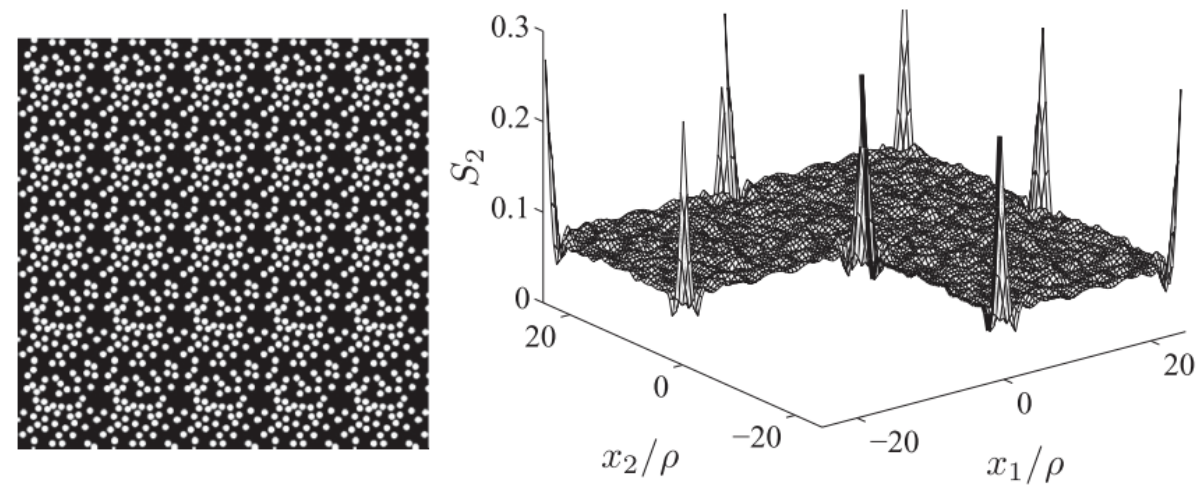

Figure 3.4: Tiled microstructure compression sample.

Representation of the tiling produced by assemblying the building blocks from the prior figure (left) and a two point probability analysis of the produced tiling depicting corner issues (right). In the two-point probability analysis, the tiling is compared to an objective function that has been previously established. Reproduced from [53] 
by Novák et al. [55] in this subject.

In their work, Novák et al. [53] focused on microstructure compression aimed at the efficient representation of material structures in multiscale computations. Their approach consisted of controlling compression algorithms in small Wang tile sets in combination with the stochastic Cohen-Shade-Hiller-Deussen (CSHD) tiling algorithm [44]. A two-dimensional square domain was discretized by a regular square lattice made up of lattice cells that contain specific morphological patterns, as seen in figure 3.3. The morphology of the tiling was analyzed using a two point probability function on the tiling. One of the challenges they found was an increase in density in corner edges, as can be seen in figure 3.4, the solution they propose is to experiment with different tile sizes and tilings and to establish edge constraints during the tile extraction phase.

The issues encountered by Novák et al. [53] were then improved upon by Doškář et al. [55]. His work presents a technique to synthesize and compress complex material morphologies. Tiles are designed following the Image Quilting Algorithm (IQA) proposed by Efros et al. [54] which allows the fusion of images without severe visual defects. The work presents a method for the representation of complex microstructural patterns by making use of Wang tile sets. His work presented the greatest contribution to this research by providing a general guideline on the assembly of complex images for the development of a Wang tiling.

\subsubsection{Wang tilings in atomistic simulation}

Sections 2.3 and 3.2.1 provide the current state of the art of atomistic simulations and Wang tiling in engineering respectively. It has highlighted the limitations associated with the creation of a metallic glass and with the implementation of Wang tilings in 
engineering.

This research is an extension of the work conducted by Novák and Doškář ( [53] and [55] respecively) for the representation of microstructures. Their work is the first to implement Wang tilings in materials engineering to reconstruct disordered microstructures and amorphous solids.

This work consists on the implementation of Wang tilings for the representation of metallic glasses, where the resulting system is known as the Wang tiling specimen. The creation of this specimen is an adaptation of the technique presented by Doškár [55]. It consists on using an Image Quilting Algorithm (IQA), proposed by Efros et al. [54], for the fusion of microstructural excerpts, that will ultimately result in Wang tiles that when assembled will yield a tiling.

These microstructural excerpts, defined as samples, are fragments taken from a reference configuration (true specimen) that is considered a metallic glass. One hypothesis is that the accuracy with which the Wang tiling specimen represents metallic glasses depends on the true specimen used and its realism. If this hypothesis proves true, smaller samples can be created and assembled for studying larger systems or, small tile sets can be studied instead of large random specimens.

However, some of the limitations associated with atomistic simulations and Wang tilings need to be taken into account. Molecular Dynamic (MD) simulations of bulk systems are nearly always conducted assuming periodic boundary conditions, ensuring there are no edges effects in a simulation. This boundary condition can be harder to ensure when working with Wang tiles. A Wang tiling is assembled ensuring edge code compatibilities between contacting Wang tiles which might not necessarily be the case when using periodic boundaries. There is no guarantee that periodic edges will be the same.

Regarding the Wang tiling specimen creation, some concerns are regarding (i) 
the creation of sample tiles and the location of atoms at the edges, (ii) the types of interatomic interactions that will take place between neighbouring tiles when the tiling is assembled, and (iii) implementing edge constrains in atomic simulations.

The value of this research is the novelty of implementing Wang tilings for MD. This is the first recorded account to date that makes use of this theory in MD simulation of metallic glasses. If the adapted technique proves successful, the computational effort for the generation of large samples of amorphous solids will be greatly reduced, allowing larger samples to be studied. It will also enable researchers to study small tile sets that can generate different tiling in each assembly. It would then be easier to generate new specimens of the same material without having to start from scratch. 


\section{Chapter 4}

\section{Methodology}

Prior chapters have provided the necessary background to understand the importance of studying amorphous solids (refer to section 2.1), the value in performing Molecular Static and Dynamic simulations for the representation of solids (refer to section 2.2), and the relevance of implementing Wang tilings for the representation of noncrystalline solids (refer to section 3.2). Chapter 3 also provided a general description on the creation of Wang tilings, their application in different fields of research and some of the issues that other researchers have faced.

One of the objectives of this research is to develop and validate a methodology that uses Wang tilings to accurately represent amorphous solids. Hence, two models were created. The first model requires the creation of an amorphous sample (random specimen) and ensuring it is in equilibrium. This model, referred to as the true specimen, will serve as both the reference configuration and as the specimen used for the creation of the Wang tiling. The second model will be the new amorphous solid created through Wang tiles (semi-random specimen), referred to as the Wang tiling specimen. This chapter includes a methodology proposal for the creation of both specimens, alongside information on problems encountered, their solutions and their impact on the methodology. 
The intent is to create Wang tiling specimens that can be used as surrogates for a true specimen. A Wang tiling specimen that is representative of a true specimen would be of great value for the development of larger systems of study. It might also enable researchers to study small tile sets instead of large systems, and create a correlation between smaller specimens and larger specimens of metallic glasses.

The Molecular Dynamics (MD) simulations were carried out using the LAMMPS simulation package [33] and the embedded-atom model potential (EAM potential) provided by Mendelev et al. [35] for a two-component metallic glass. For MD and EAM potential information refer to chapter 2 .

Our working material is a two-dimensional, two-component, non crystalline solid. It is composed of copper $(\mathrm{Cu})$ and zirconium $(\mathrm{Zr})$ molecules in a 64:36 ratio approximately. The two-component system that was chosen has been the subject of other investigations $[35,56,57]$. The simulations were modelled in two instead of three dimensions because most literature on Wang tilings is two-dimensional, future work can seek to extrapolate the findings of this work to three-dimensions and the implementation of Wang cubes. The use of two-dimensional systems also aids in understanding more complex systems that are representative of three-dimensional materials.

It is important to note that a smaller true specimen was created as a visual aid for this chapter. This smaller specimen was only used for visualization purposes and was not taken into account when generating quantitative data. Those figures that are created from this smaller true specimen state this in their caption.

\subsection{True specimen}

An amorphous $\mathrm{Cu}_{64} \mathrm{Zr}_{36}$ sample with a total number of 16,000 atoms was developed. The creation of this true specimen was all performed using the LAMMPS simulation 
package. The methodology consists of 4 steps which result in the random system defined as original specimen:

1. Creation of an initial configuration

2. Energy minimization of the initial configuration

3. Heating and quenching of the system

4. Energy minimization of the system after quenching.

The first step consisted on the creation of a two-dimensional simulation box of a user specified length that was empty. The box was then filled with particles of $\mathrm{Cu}-\mathrm{Zr}$, ensuring a 64:36 ratio. The initial coordinates of the particles was randomly determined through one of LAMMPS's features. The system's boundaries were considered periodic in all directions (section 2.2.2). Even though atom interactions would only be accounted for in two-dimensions, there would be no force calculations in the $\mathrm{z}$ direction and atoms were constrained to move only in the xy plane.

The first step results in a two-dimensional, two-component, periodic, amorphous solid with a 64:36 composition ratio, in which atoms may be too close together, generating high energy and forces. This is because the random generator does not take into account interatomic distances when placing atoms. This system is not at a local energy minima and cannot be considered representative of metallic glasses. An energy minimization is therefore required before performing MD. The initial system was equilibrated first at a fixed volume, and then under zero pressure. These minimizations lead to a densely packed random atomic arrangement that had reached an energy minimum.

Once the initial system had reached or was closer to a local energy minima, it was heated to $2250 \mathrm{~K}$ to ensure the system is above its glass transition temperature 


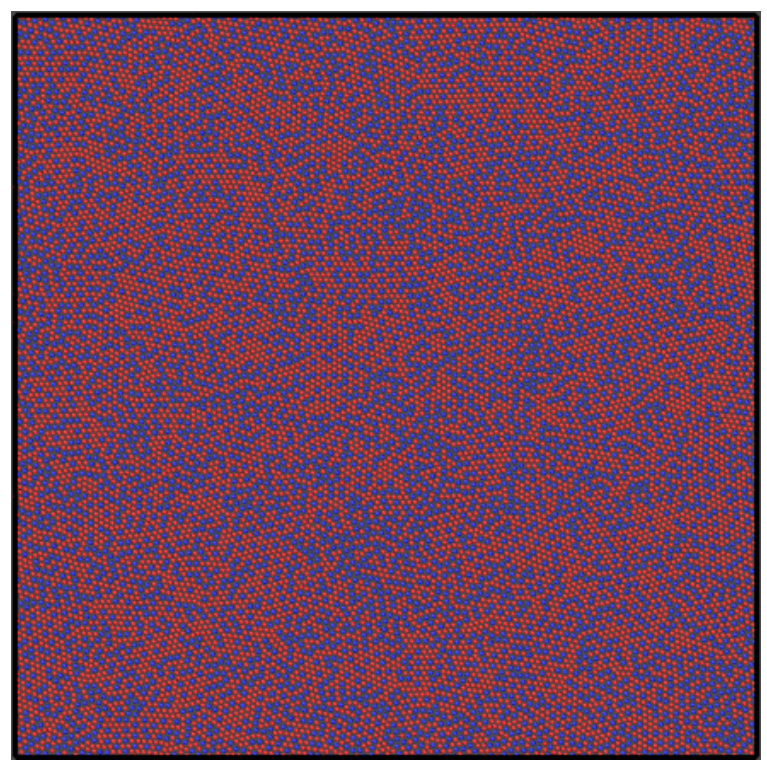

Figure 4.1: True specimen.

Representation of the resulting periodic two-dimensional two-component specimen, the colours represent $\mathrm{Cu}$ or $\mathrm{Zr}$ atoms (red and blue respectively).

(refer to chapter 2 on the importance of the glass transition temperature). It was then equilibrated for 2 nanoseconds (2,000 timesteps) and then quenched to $0 \mathrm{~K}$ using a temperature damping parameter of 20 nanoseconds (the temperature changes from $2250 \mathrm{~K}$ to $0 \mathrm{~K}$ in 20 nanoseconds or 20,000 timesteps).

Since quenching does not ensure the system is at an energy minimum, the final step is to relax the system. The system was allowed to relax first at a fixed volume and then at zero pressure; this ensured the system had no lingering residual forces that might affect the deformation analysis. The resulting system after these steps is considered the true specimen, see figure 4.1 .

The steps used for the creation of the true specimen, that were previously described were the end result of several iterations. In section 3.2 the sensitivity of metallic glasses to the initial configuration, their temperature dependence, and simulation properties were presented. Several concerns arose during the different stages; 
some of these concerns were to:

1. Determine the existence or non-existence of a system dependency on the random seed used to generate the initial configuration.

2. Ensure the system reached a local energy minima before and after quenching.

3. Determine whether there are differences (in composition or behaviour) amongst different specimens created using the same methodology.

The process was repeated several times with different parameter values to ensure the creation of an equilibrated true specimen. Some of the parameters modified were the timesteps used in minimization, heating or quenching, the temperature damping parameter, the maximum heating and minimum quenching temperatures, and the seed used in the algorithm that creates the first configuration from scratch. These simulations provided answers to the concerns mentioned previously.

1. There was a system dependence on the initial configuration that was randomly generated by LAMMPS. Some initial configurations would present atoms in close proximity or overlapping, in these systems atoms would not be able to move freely in the simulation box during heating and quenching. Performing an energy minimization and allowing system's volume to change solves this issue.

2. Using the wrong system density can result in phase segregation and a low atomic density in nucleation, as seen in figures 4.3 and 4.2 respectively. Both situations lead to systems that are not representative of metallic glasses. 


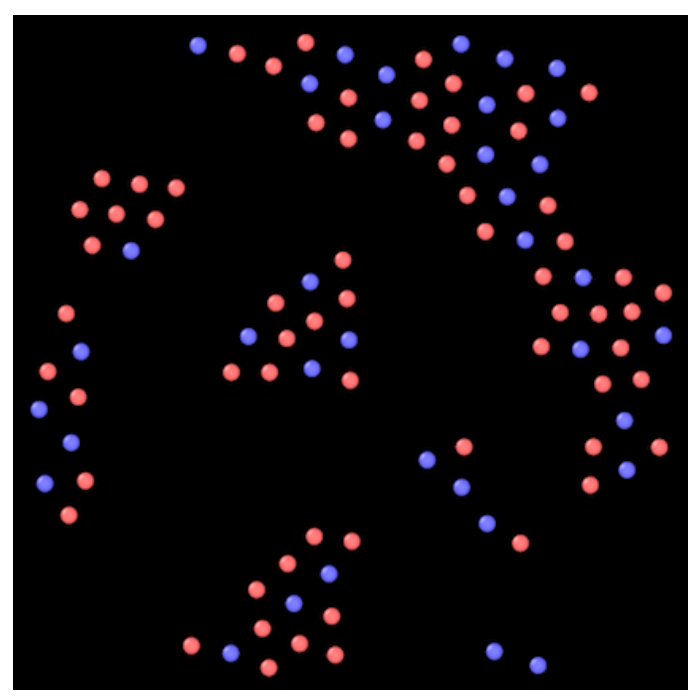

Figure 4.2: Specimen nucleation during minimization due to low initial density.

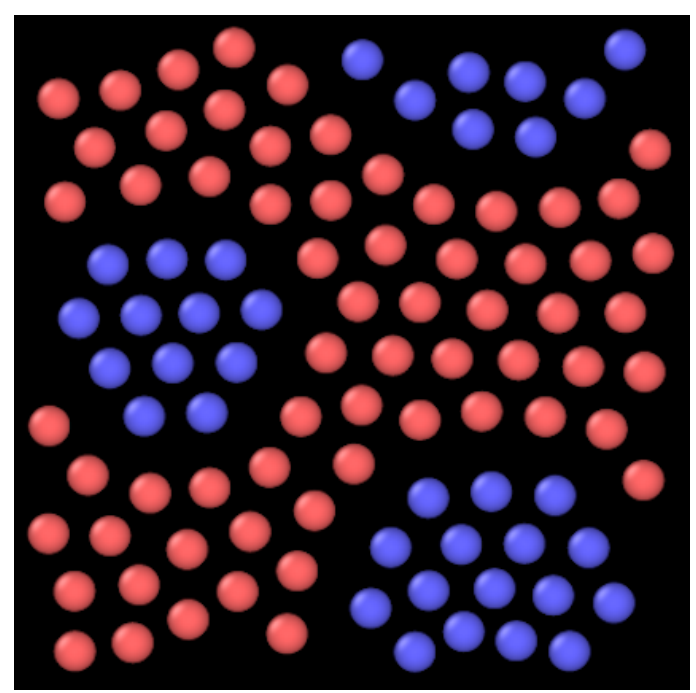

Figure 4.3: Phase segregation of particles during minimization due to low initial density.

The final specimens that were physically realistic representations of metallic glasses were those that were permitted to expand or contract at zero pressure, allowing the system to reach the correct atomic density.

3. In order to ensure that the final specimen that was created was representative of metallic glasses and that the methodology implemented did not yield significant differences, several specimens were created from different initial configurations. To verify that all specimens were homogeneous and representative of the same metallic glass, a quantitative comparison of their mechanical properties was performed. The bulk and Young's modulus for all specimens was determined with some differences between specimens (specially in the Young's modulus), see table 4.1 for the resulting values. This guaranteed that the methodology used led to a similar energy minimum and that all specimens could be considered to be representative of the $\mathrm{Cu}-\mathrm{Zr}$ metallic glass.

The Young's modulus was determined by submitting the specimen to a uniaxial 
change in length and studying it's reaction, where the Young's modulus is the gradient of the stress-strain graph and is the inclinations of the tangent as seen in figure 4.4.

Table 4.1: Elastic constants for eight different specimens, where the Bulk and Young Modulus are measured in $\mathrm{eV} / \AA^{2}$.

\begin{tabular}{|c|c|c|}
\hline Sample & 2D Bulk Modulus & 2D Young Modulus \\
\hline 1 & 46.45 & 385.53 \\
\hline 2 & 42.00 & 373.13 \\
\hline 3 & 44.85 & 378.54 \\
\hline 4 & 42.50 & 382.64 \\
\hline 5 & 41.53 & 375.97 \\
\hline 6 & 44.25 & 391.84 \\
\hline 7 & 40.17 & 411.31 \\
\hline 8 & 43.00 & 404.21 \\
\hline Average & $43.09 \pm 1.57$ & $387.89 \pm 10.92$ \\
\hline
\end{tabular}

Quenching proved to be a trial and error based approach that could be limiting for later stages of the investigation. The establishment of an adequate cooling rate determines whether one will see the creation of a metallic glass or a homogenous flowing system (system where each volume element undergoes the same strain rate and behaves like a Newtonian fluid [58]), where the desired state is a solid. A fast cooling rate would skip the crystallization phase and would result in homogeneous flow; a very slow cooling rate could result in phase segregations or nucleation of the system [1]. After ensuring the system underwent crystallization, a second energy minimization was performed on the system at a fixed volume and at zero pressure.

The end result of the iterations was the establishment of the four steps for the 


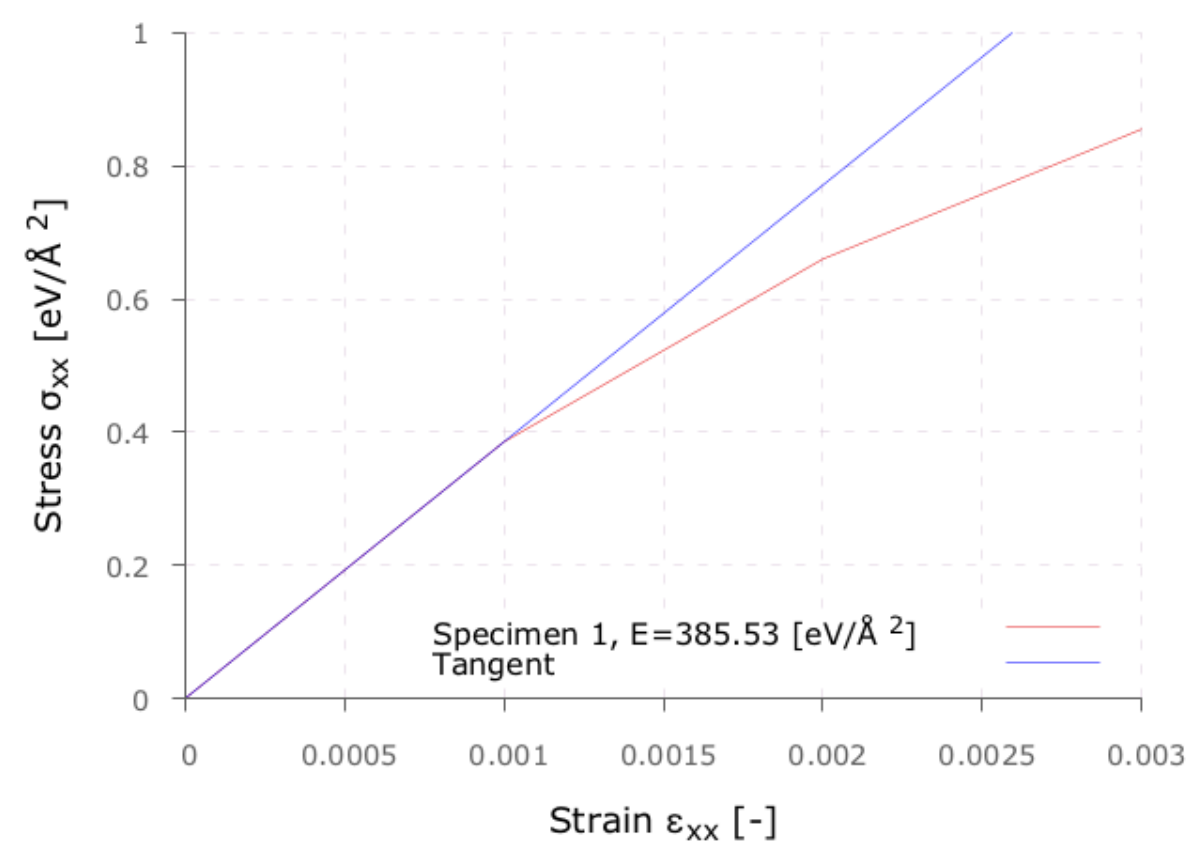

Figure 4.4: Graphic determination of the Young modulus.

The slope of a tangent line to the stress-strain curve of a specimen, in the vicinity of zero, is considered to be the Young modulus's value. The figure shows the Young modulus calculation for one of the ten true specimens that were created.

creation of a two-dimensional, two-component, homogeneous metallic glass, known as the true specimen, that has reached an energy minimum and can be considered to be in equilibrium. This specimen is considered a random system as it was formed from a randomly generated initial configuration.

\subsection{Wang tiling specimen}

In Chapter 3 the history, application and fundamentals on Wang tilings were introduced. This section includes the establishment of a methodology for the creation of Wang tilings representative of metallic glasses, that can be used in molecular dynamics simulations.

In general, the creation of a tiling involves the construct of smaller basic elements, 
such as prototiles and Wang tiles that will form a tile set, the assembly of the Wang tiles will produce the final Wang tiling specimen. A description on all the stages of the methodology, suggestions and solutions to some problems is presented in the subsequent pages. While there might be other paths that can be used for the creation of a Wang tiling specimen, the methodology presented encompasses the general steps used in other fields and produces tilings representative of two-dimensional metallic glasses; ideas on future research on the topic will be presented in chapter 6 .

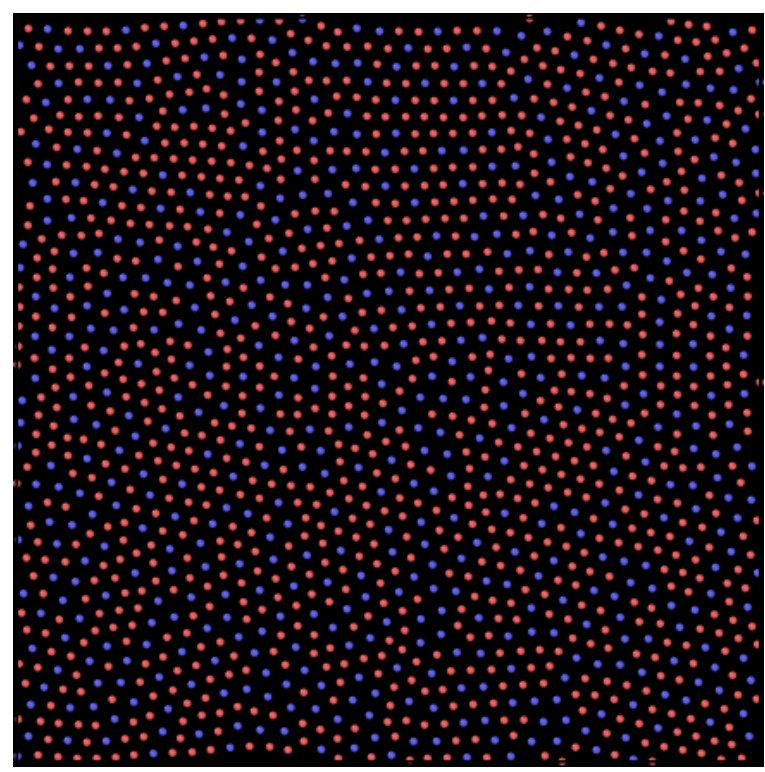

Figure 4.5: Smaller true specimen.

The purpose of the smaller true specimen is to better illustrate the steps in the creation of a Wang tiling. The true specimen is composed of 1600 atoms with a $64 \mathrm{Cu}: 36 \mathrm{Zr}$ composition ratio and is 10 times smaller than the actual true specimens developed.

The development of a stochastic Wang tiling specimen consists of four stages: (i) extraction of samples from a true specimen (see figure 4.5 for an example of a smaller true specimen), (ii) creation of prototiles from the extracted samples, (iii) creation of individual Wang tiles and establishment of a tile set, and (iv) assembly of tiles into a tiling. The Wang tiles used are taken from prototiles which are assembled sections from a reference configuration that in this research is the true specimen that 
was previously generated in section 4.1. The Wang tiling specimen is considered a semi-random system as it is formed from samples of a random system (true specimen). 


\subsubsection{Sample extraction}

In this methodology, prototiles are designed based on image fusion technology that has been useful in computer graphics $[44,59]$. The design has been extracted from the Image Quilting Algorithm (IQA) designed by Efros et al. [54] for the fusion of sample images without severe visual defects. Their work consisted on the creation of small samples that were extracted from a reference configuration. These samples were then assembled following an IQA that would place images together based on probability and statistical information. The same principle was extrapolated for the construction of a metallic glass Wang tiling specimen.

The Wang tiling specimen is developed using python code that was written in collaboration with Doškář from the Czech Technical University in Prague [55].

This first step involves the extraction of samples from the true specimen, also know as the reference configuration. Eight square samples of a fixed dimension were extracted from the true specimen, as seen in figure 4.6, where its length is determined by the user. A sample is considered to be a region within the true specimen's simulation box; the sample cannot be bigger than the specimen used or fall outside of it. The location of the samples that are generated is randomly determined and leads to homogeneous samples that are geometrically identical, they are all squares of the same size.

The extraction location is randomly determined making use of a seed that is also randomly generated, these parameters ensure that tilings from different regions are extracted in different runs. For each run, different samples would be extracted and this could potentially lead to different Wang tilings.

There are possible variations in this stage that can be of interest or make significant differences in the creation of a Wang tiling, such as: 


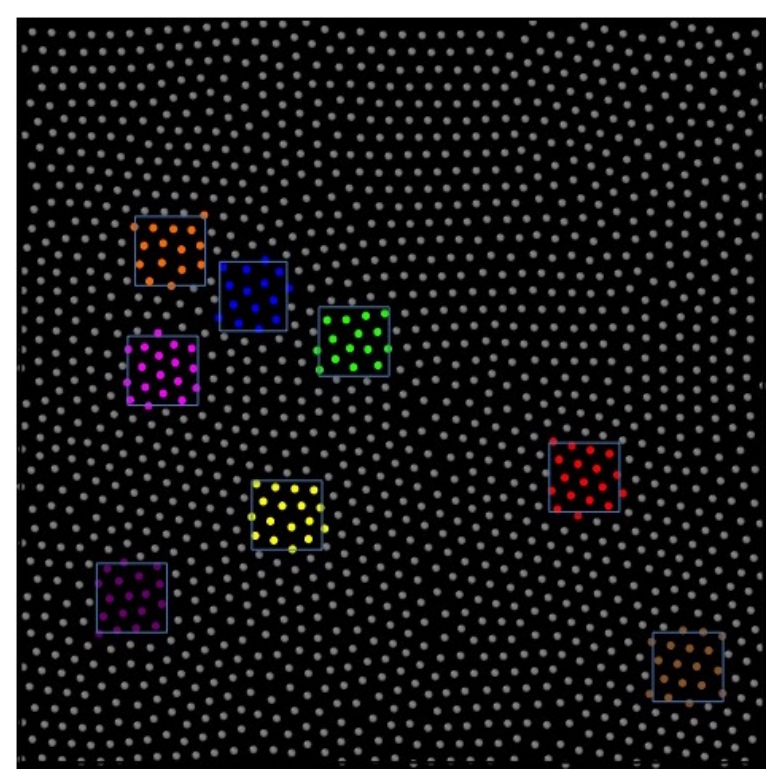

Figure 4.6: Square samples extracted from a true specimen.

The size of the samples extracted from a true specimen are user specified; in this case, they are $1 / 10$ th of the true specimen's length. The white particles in the figure represent unused particles, and coloured particles represent used particles. The different colours represent the different samples created. The extracted samples are all squares, that are hard to appreciate in the image as some samples do not seem to follow a square pattern when the atoms are very close to the edge. Image developed from the smaller true specimen.

1. Changing the size of the samples extracted.

The sample generated is only constrained to be of a geometrical shape and dimension, particle locations are not taken into account when creating it. This means, that atoms might be placed along the edge or in very close proximity to the edge.

Both cases are of interest when working with atomistic simulations, MD studies the evolution of atom interactions, where these interactions are a function of interatomic distances, see section 2.2 for more information on MD. Therefore, considering that the next stage in the development of a Wang tiling is the creation of a prototile, which is the assembly of samples, the location of these edging atoms might be influential. 
The size of the samples extracted would also impact the number of tiles needed to tile a lattice of area $L \times L$, if the area is very large, it might be beneficial to use big tiles instead of small tiles.

2. Adding more constraints for sample extractions.

If more constraints were enforced during sample extractions, cases such as atom

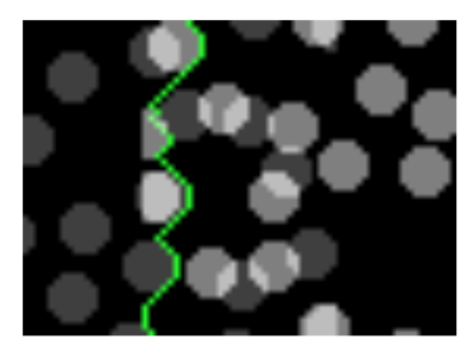

Figure 4.7: Modified Image Quilting Algorithm (IQA)

The edge of the extracted sample is winding instead of a fixed square shape. Reproduced from [60].

overlapping, small interatomic distances or large vacancies would be avoided.

The IMQ algorithm that was created by Efros et al. [54] used non symmetrical images that were geometrically similar to squares. Since they were working with images, they made a pixel density analysis to determine the path the edge should follow which results in a labyrinth path being followed as seen in figure 4.7. This ensured that there was a greater diversity in the edges so that they would look more homogeneous during assembly. Examples of the implementation of this modified IMQ algorithm can be found in [60].

A similar algorithm can be developed for working with metallic glasses. This would enable the creation of samples that during assembly might have more compatibility and exhibit less defects (which will be explained in the next section). This variation in particular was not explored during this research, but might be interesting to explore at a later date. 
Table 4.2: Wang tile set composed of 16 distinct Wang tiles. Tiles are created by placing four different samples in a specific orientation (NSEW).

\begin{tabular}{|c|c|c|c|c|}
\hline & \multicolumn{4}{|c|}{ Orientation } \\
\hline Tile & N & S & E & W \\
\hline 1 & 1 & 1 & 3 & 3 \\
\hline 2 & 1 & 1 & 3 & 4 \\
\hline 3 & 1 & 1 & 4 & 3 \\
\hline 4 & 1 & 1 & 4 & 4 \\
\hline 5 & 1 & 2 & 3 & 3 \\
\hline 6 & 1 & 2 & 3 & 4 \\
\hline 7 & 1 & 2 & 4 & 3 \\
\hline 8 & 1 & 2 & 4 & 4 \\
\hline
\end{tabular}

\begin{tabular}{|c|c|c|c|c|}
\hline & \multicolumn{4}{|c|}{ Orientation } \\
\hline Tile & $\mathbf{N}$ & $\mathbf{S}$ & $\mathbf{E}$ & $\mathbf{W}$ \\
\hline 9 & 2 & 1 & 3 & 3 \\
\hline 10 & 2 & 1 & 3 & 4 \\
\hline 11 & 2 & 1 & 4 & 3 \\
\hline 12 & 2 & 1 & 4 & 4 \\
\hline 13 & 2 & 2 & 3 & 3 \\
\hline 14 & 2 & 2 & 3 & 4 \\
\hline 15 & 2 & 2 & 4 & 3 \\
\hline 16 & 2 & 2 & 4 & 4 \\
\hline
\end{tabular}

\subsubsection{Prototile assembly}

The reference samples that were previously extracted are then assembled to create aggregates of four, as seen in figure 4.8. The use of a diamond pattern is so that when we cut out a square tile, the region near the edges will be close to the correct relaxed configuration after the tiling assembly. The samples are placed accordingly to the edge codes of the tile to be produced, following the tile set table (table 4.2). In this research 16 Wang tiles were created from 4 samples; the 16 tiles created account for all possible combination configurations of the tiles, as can be seen in table 4.2. Out of the 8 samples that were extracted, only 4 were used to create a tiling, hence, using different samples might lead to different tiles and tilings.

Atomic simulations are dependent on the interatomic interactions taking place between particles, refer to chapter 2 . One of the problems that might arise in the edges is: atoms overlapping, being located very close together or the lack of atoms (gaps) in 

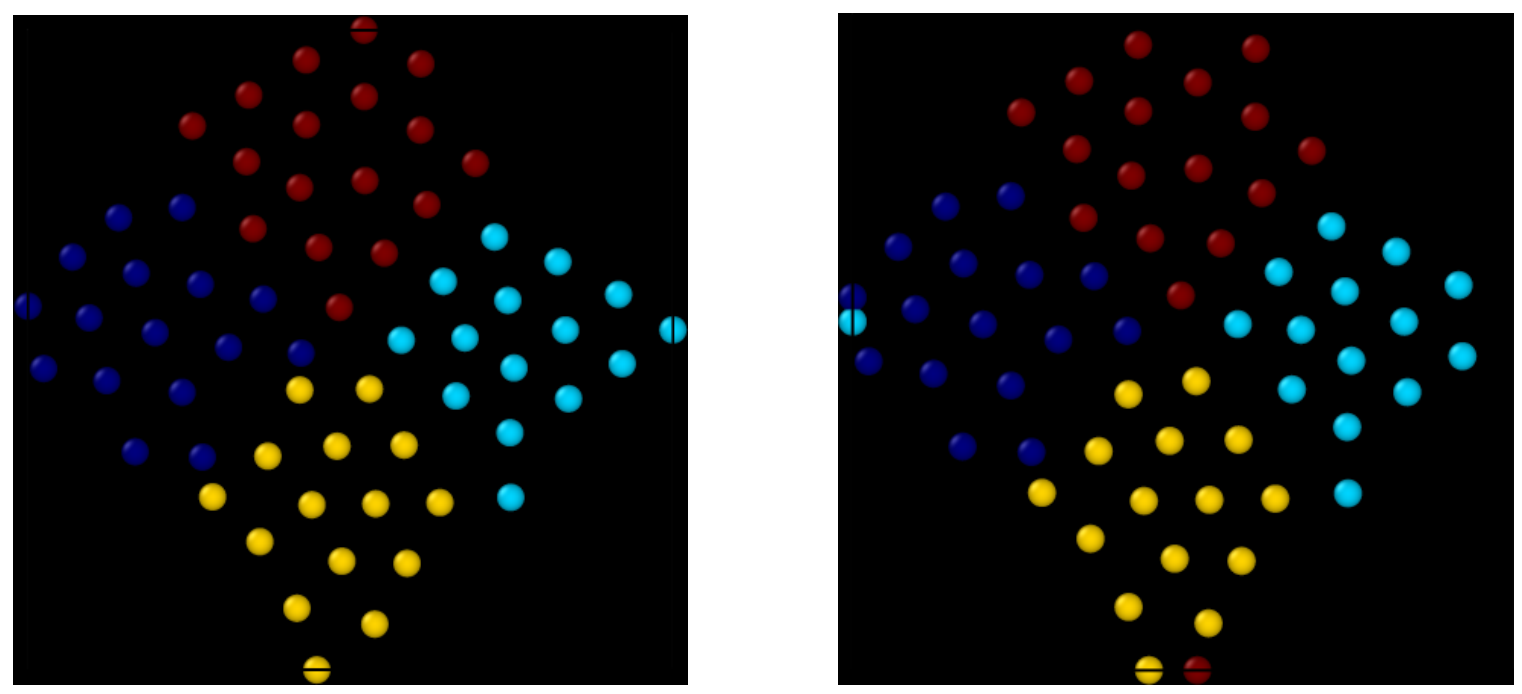

Figure 4.8: Prototile before and after minimization

The prototile to the left shows an assembly of four different samples before being minimized and the one to the right after minimization. Both images were developed from the smaller true specimen.

some regions. Figure 4.8 shows an example of atoms being in close proximity before minimization (centre of the prototile) that gets fixed during minimization. Figure 4.9 is an example of regions with few atoms. These situations are problematic because they create high energy zones and the latter a low density region; all of which are more susceptible to undergo inelastic deformations and might cause simulations to yield data that is not representative of a stable system and reality. There are several options for verifying that none of those situations is occurring:

1. Manually check that none of the prototiles assembled have these situations. A qualitative analysis can be done by visualizing the assembled prototiles through Ovito [34]. The user can visually determine whether several prototiles experience the same phenomena and can determine the specimen(s) where these issue arises. Changing the specimen to one of the other four that weren't used might yield to prototiles without these "defects". However, there is no guarantee that none of the "defects" will present themselves across prototiles regardless 
of the specimens used.

2. Perform several iterations of the extraction process and use samples that do not have atoms near the edges.

This idea is similar to the previous one and would also ensure that fewer defects are present during the prototile assembly. This solution can be time consuming and requires the user to visually determine which samples can be used and which cannot.

3. Perform selective sample extractions ensuring atoms are not located close to the edge.

This approach was briefly described in the previous subsection, during the creation of the samples. It was not explored during this research.

4. Calculate the distance between atoms $j$ and $i, d_{j i}$, and when $d_{j i}<r_{\text {min }}$, delete atom $j$ ( $r_{\min }$ is the minimum distance expected between atoms).

5. Perform a "constrained energy minimization" on all the prototiles simultaneously and allow the system to reach a stable state (close to equilibrium) where these situations are reduced or nonexistent.

In this research, a constrained energy minimization refers to an energy minimization where atoms from the same sample move simultaneously across all prototiles. Therefore, sample_i would look exactly the same in all prototiles that include it (where i can be 1 to 4 ).

Greater interest was placed in the last three solutions. In order to ensure that the prototiles were close to equilibrium, two sub-steps were explored for atoms in close proximity. First, if atom $j$ and atom $i$ were overlapping or very close together, and the distance between them was smaller than an accepted minimum, $d_{j i}<r_{\text {min }}$, atom 


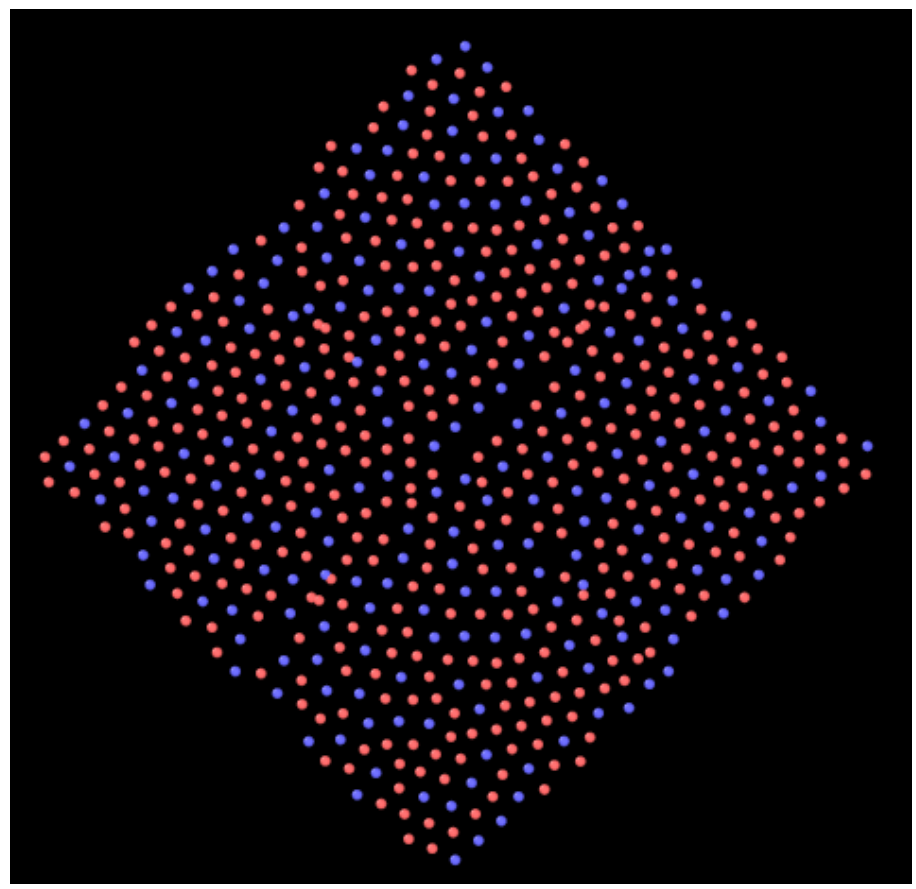

Figure 4.9: Prototile issues.

The prototile presents areas where atoms are missing (low density regions) and areas where atoms are in close contact or overlapping (high density regions). Image developed from a true specimen.

$j$ would be deleted across all prototiles. Second, a constrained energy minimization was attempted on the system, seeking to ensure that atom displacement for atom $j$ would be identical in all prototiles.

Both sub-steps presented limitations. When working with big systems, determining $d_{j i}$ was time consuming and slow because the distances between all atoms would need to be determined for all prototiles, and all atoms that needed deletion would need to be deleted from all prototiles to ensure edges remained identical.There is no guarantee that only atom $j$ would be deleted in each prototile as atom $i$ might be in close proximity to another atom in another prototile assembly, hence, regions with no atoms might occur occasionally (low density and high energy).

The second sub-step was used as a solution for the occasional low density regions that were created through atom deletion, however, this step proved to be even more 
limited and challenging than the first.

Wang tile sets are composed of coded tiles with identical edges, refer to chapter 3 for examples. Each edge is attributed a number or colour for tracking purposes and its constituent particles are identical in all tiles. This means that if atom $i$ moves from $(0,0)$ to $(0,2)$ in tile 3 , the same movement needs to occur in all tiles where the atom is located. At the prototile level, this means that all atoms need to move simultaneously across all prototiles during an energy minimization (constrained energy minimization).

Ensuring the movement of all atoms in the identical manner when performing molecular dynamics is challenging if the atoms are not bound to one another and have different neighbours in the prototiles. LAMMPS is able to implement this constraint with groups of atoms (molecules), but not with individual atoms, therefore a constrained energy minimization on all prototiles can be performed using small clusters of atoms. Performing the energy minimization on all prototiles at the same time was very time consuming and clusters moved independently in the edges, therefore edges still presented differences.

Due to the second sub-step's limitations, an alternative energy minimization was implemented that yielded a prototile close to equilibrium. Instead of performing an energy minimization on all prototiles simultaneously, the energy minimization was performed independently on individual prototiles.

In summary, 16 prototiles were created through the assembly of 4 samples of equal dimensions. Sample assembly depends on the tile combination that will be created which follows the tile set combinations from table 4.2. After assembly, atoms might be in close proximity and lead to high energy regions susceptible to fracture. Selective deletion of atoms across prototiles can reduce high energy regions but might lead to low energy regions if too many atoms are deleted. An energy minimization 
with constraints can lead to a new minimized prototile but using too small regions might mean differences in same edges from the same sample, in order to avoid this, prototiles get individually minimized. These last steps can be avoided if the initial prototile assembled does not have close-contacting atoms, hence, a more efficient sample extraction method might be more beneficial.

\subsubsection{Wang tile construction}

Wang tiles are a square tile that has been cut out from the prototiles previously assembled, see chapter 3 for the definition of a Wang tile. Edge codes are determined by the sample used in the prototiles assembly and are determined by the unique combination required to form a tile set as can be seen in table 4.2.

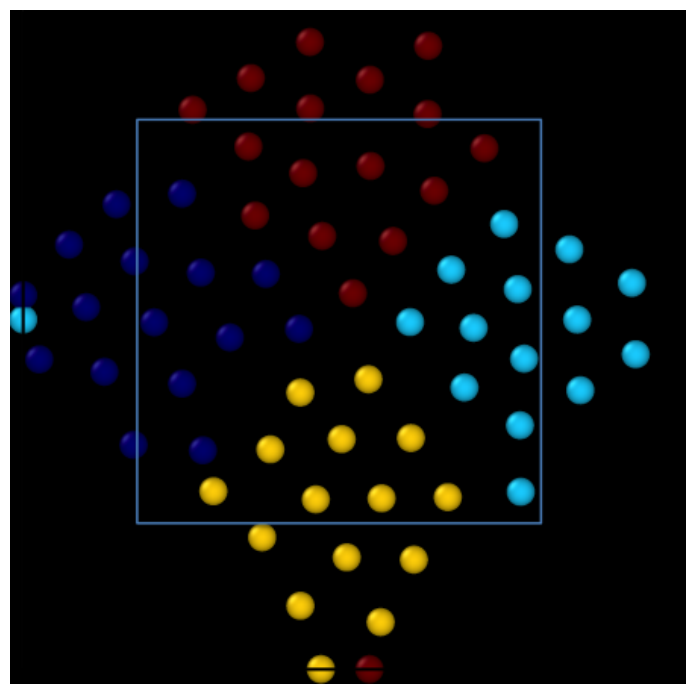

Figure 4.10: Wang tile representation inside a prototile.

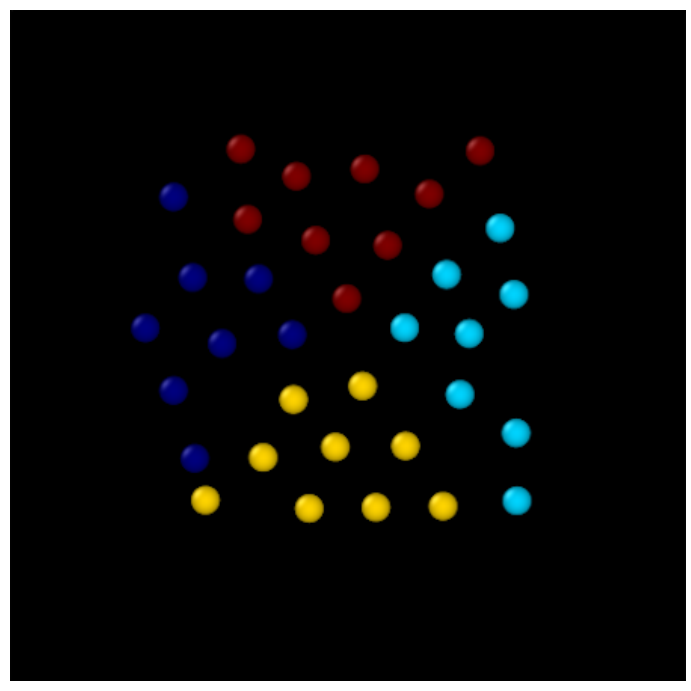

Figure 4.11: Wang tile extracted from a prototile.

Wang tile formed from cutting a $45^{\circ}$ square of length $l_{e}$ from the prototile, where $l_{e}$ is user specified and must fall within the prototile. The purpose of creating a tile using a $45^{\circ}$ rotation from the prototile, is to ensure the edges of the Wang tile are close to an energy minimum. These new edges (except for the corners) are less likely to exhibit missing atoms, atoms in close proximity or overlapping. Image developed from the smaller true specimen. 


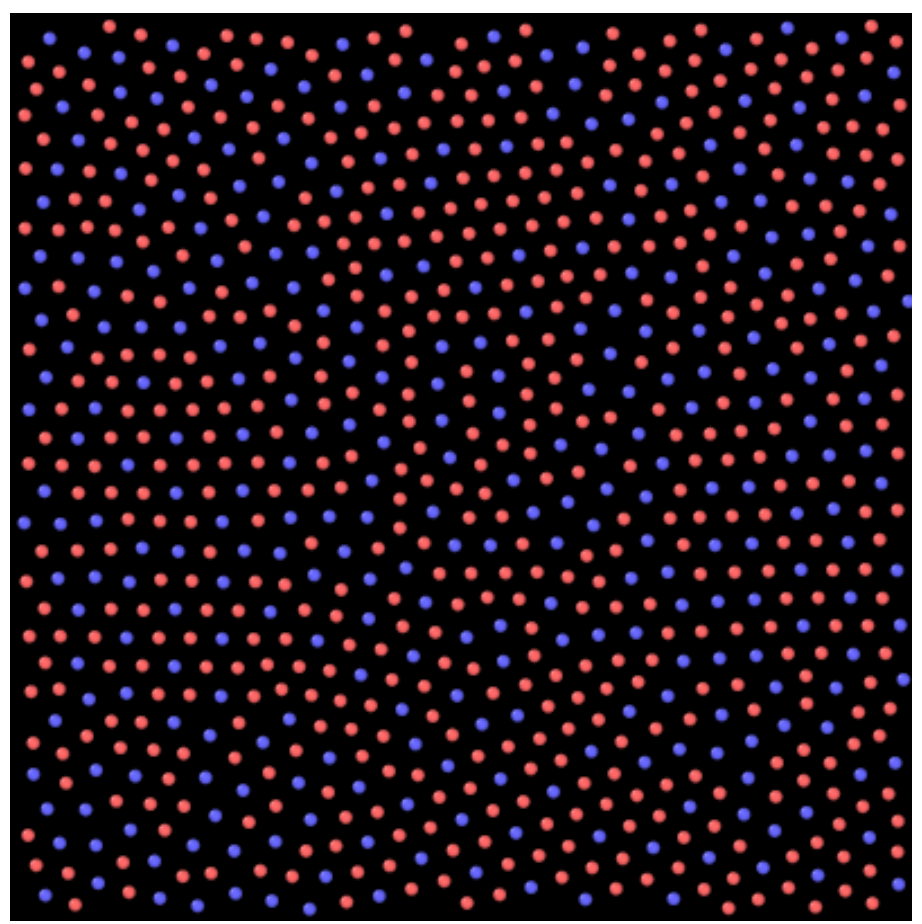

Figure 4.12: Wang tile produced from a minimized prototile.

In the prior step, prototile assembly, the possible existence of three defects was presented. These defects (overlapping, high density regions and low density regions) are present in regions where the samples come into contact and are not present in the middle regions. Therefore, Wang tiles are formed by cutting a $45^{\circ}$ square of length $l_{e}$ from the diamond shaped prototile, see figures 4.10 and 4.11 for an example on the creation of a Wang tile from a prototile. This ensures that the edges of the Wang tiles formed are relaxed and remain relaxed with neighbouring tiles when the tiling is assembled. The size of the tile created is conditioned to fitting diagonally inside any given prototile and therefore needs to satisfy $l_{e}<\sqrt{2} l$, where $l$ is the length of the prototile.

The continuity of the tile is ensured because the cut is taken diagonally across the sample, and because the same sample is used for all edges sharing the same code, represented in the figures by the different colors (see figure 4.11 for an example). 
Performing a diagonal cut keeps prototile defects confined mainly to the interior of the tile; the region that does not need to match across the tiling. If the prototiles used do not have any defects or they were corrected in the prior stage, the tile should look homogeneous and with no visible overlapping or high/low density regions, as seen in figure 4.12 .

\subsubsection{Wang tiling assembly}

Once a tile set is developed, the last step is to assemble the tiles and generate the Wang tiling. The tiling is assembled in row by row order, so that the edge codes of a newly placed tile comply with those of its neighbours placed beforehand, see figure 4.13 and chapter 3 for other visual aids. The end result is a square tiling of size $\left(N * l_{e}\right)$, where $N$ is the number of tiles to be assembled in either the $\mathrm{x}$ or y direction and $l_{e}$ is the length of the Wang tiles that conform the tile set.

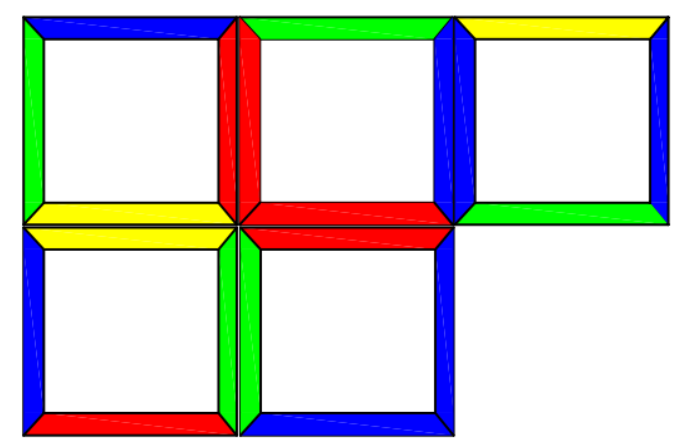

Figure 4.13: Wang tile assembly.

Wang tiles are assembled ensuring edge code compatibility. Interacting tiles have the same edges (same colour). The resulting system after assembly is the Wang tiling.

As a final step in the creation of a Wang tiling specimen, the assembled tiling is submitted to an energy minimization without any constraints. The specimen is 
allowed to relax first at fixed volume and then at zero pressure until it reaches a relaxed state. The end result is a system that is at an energy minimum and can be compared to the original specimen but cannot be considered a full tiling as some tile identities might have been. Figure 4.14 shows the $\eta_{i}^{\text {Mises }}$ values for a tiling after minimization, specifics on how this value is determined are found in chapter 5 . The image is created by comparing the resulting Wang tiling specimen after undergoing the energy minimization to the original assembled tiled system (before minimization), from the image, we can determine that some regions suffer greater displacements than other and that these tend to be where different tiles come into contact.

The result is the establishment of a methodology for the implementation of the Wang tiling theory developed by Wang in 1961 [8]. A semi-random, two-dimensional, two-component metallic glass is formed, known as the Wang tiling specimen. The specimen is created from small samples taken from a random true specimen that is in equilibrium and is considered representative of metallic glasses. There are several concerns that need to be kept in mind during the creation of a Wang tiling specimen, these concerns can condition the physical characteristics of the glass. 


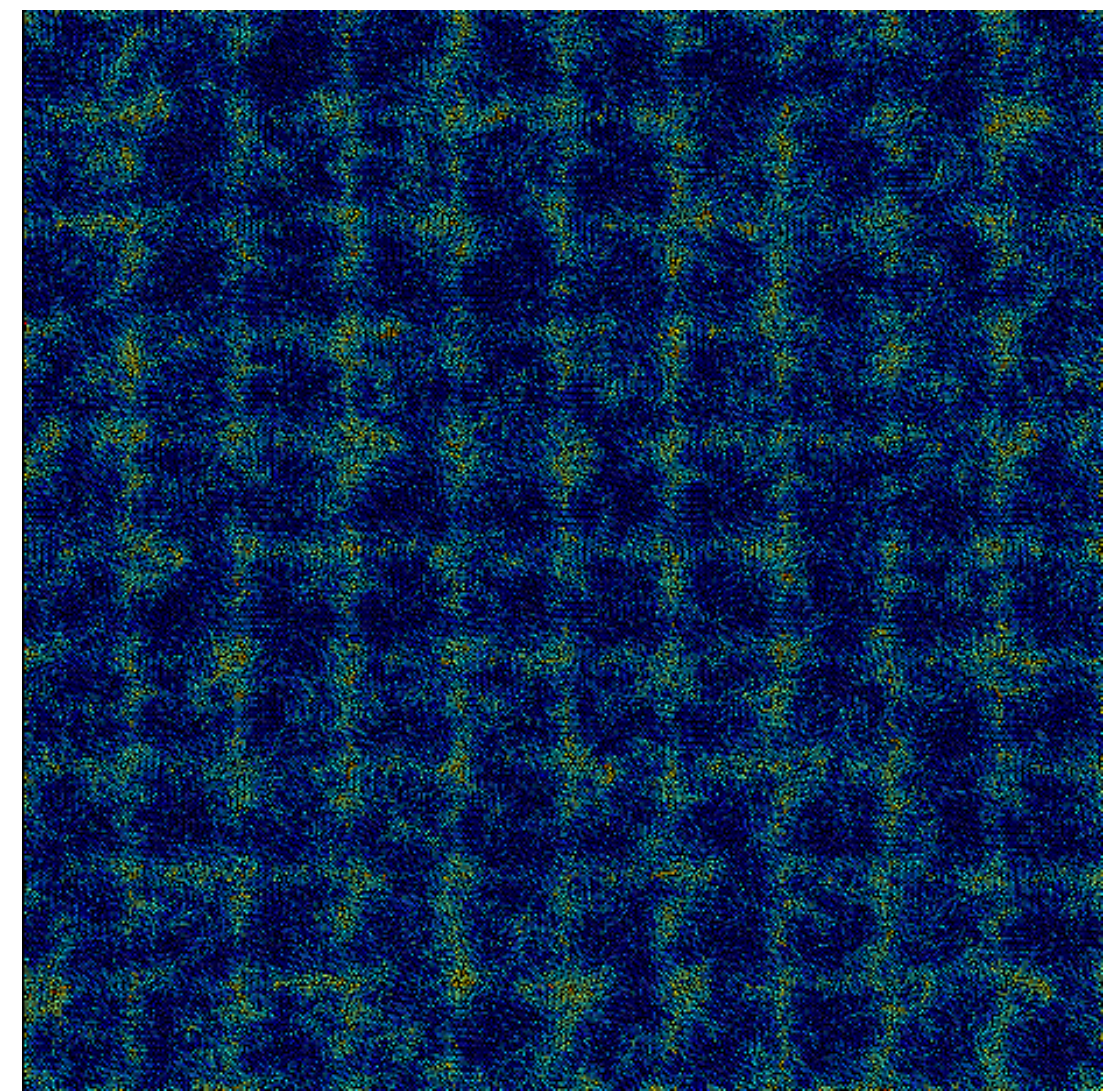

Figure 4.14: Local atomic Mises strain, $\eta_{i}^{\text {Mises }}$, analysis of a Wang tiling specimen after minimization.

In order to quantify the changes experienced by a tiling specimen before and after minimization, the atomic local Mises strain is determined for the system after minimization. The regions that experience the greatest amount of change appear to be located where the different tiles come into contact, creating the effect of a checkered specimen. The image also highlights the corners where four tiles come into contact as being the ones with most change as there is a higher concentration of affected atoms in these regions. Atoms are coloured according to their $\eta_{i}^{\text {Mises }}$ value, ranging from $0.02-0.2$, and $\eta_{i}^{\text {Mises }}{ }_{\text {max }}=0.2675$ 


\section{Chapter 5}

\section{Results Analysis}

The scope of this chapter is the macroscopic and microscopic comparison of a randomly generated true specimen and a Wang tiling specimen. Reiterating, the working material is a two-dimensional, two-component, non crystalline solid in which the molecules interact via the embedded atom model potential, also known as EAM potential.

For comparison purposes, both the true specimen and the Wang tiling specimen were subjected to uniaxial tension and their deformation characteristics were then studied at the macroscopic and the microscopic level. The two-dimensional simulations revealed a variety of behaviours that other researchers have reported for two-dimensional metallic glasses; refer to chapter 2 for information on some of these investigations. Specifically, we can observe reversible elastic deformation at small

applied stresses, irreversible elastic deformation at larger stresses and a strong dependence on the state of the system.

Section 5.1 measures the stress-strain properties of both models at a macroscopic scale. Section 5.2 looks at the microscopic properties of the system and its reaction to the applied stresses through an atomic local strain observation model [17]. The final section in this chapter is the results analysis of both the macroscopic and microscopic 
observations.

In this chapter, the terminology used to reference both models will be the true specimen and the Wang tiling specimen. The true specimen refers to the equilibrated specimen that was generated from a random atomic configuration after heating, quenching and performing an energy minimization. The Wang tiling specimen (semi-random system) refers to the specimen that was generated from a set of Wang tiles (extracted from prototiles assembled from the true specimen) that then underwent an energy minimization. On occasion the term specimen will be used to reference both the true specimen and the Wang tiling specimen.

Both specimens were subjected to the same mode of deformation. All the simulations were carried out at zero pressure and specimens were subjected to uniaxial tension. The applied strain-rate tensor was constrained to be symmetric in order to avoid physical rotations of the cell. Because the simulation cell is periodic, the application of uniaxial tension to the system does not create surfaces that might enhance system-size effects and interfere with bulk properties.

\subsection{Macroscopic observations}

The first observation made is of stress versus strain, at a constant strain rate. The specimens were subjected to an engineering strain rate, $\dot{\varepsilon}$, of 0.01 [1/picosecond]. The

box length $L_{b o x}$ will change as a function of time depending on the strain rate as seen in equation 5.1, where $L_{b o x}^{0}$ is the original box length and $t$ is the elapsed time. Thus, for $\dot{\varepsilon}=0.01$, the box will expand by $1 \%$ of its original length every picosecond.

$$
L_{b o x}(t)=L_{b o x}^{0}(1+\dot{\varepsilon} * t)
$$



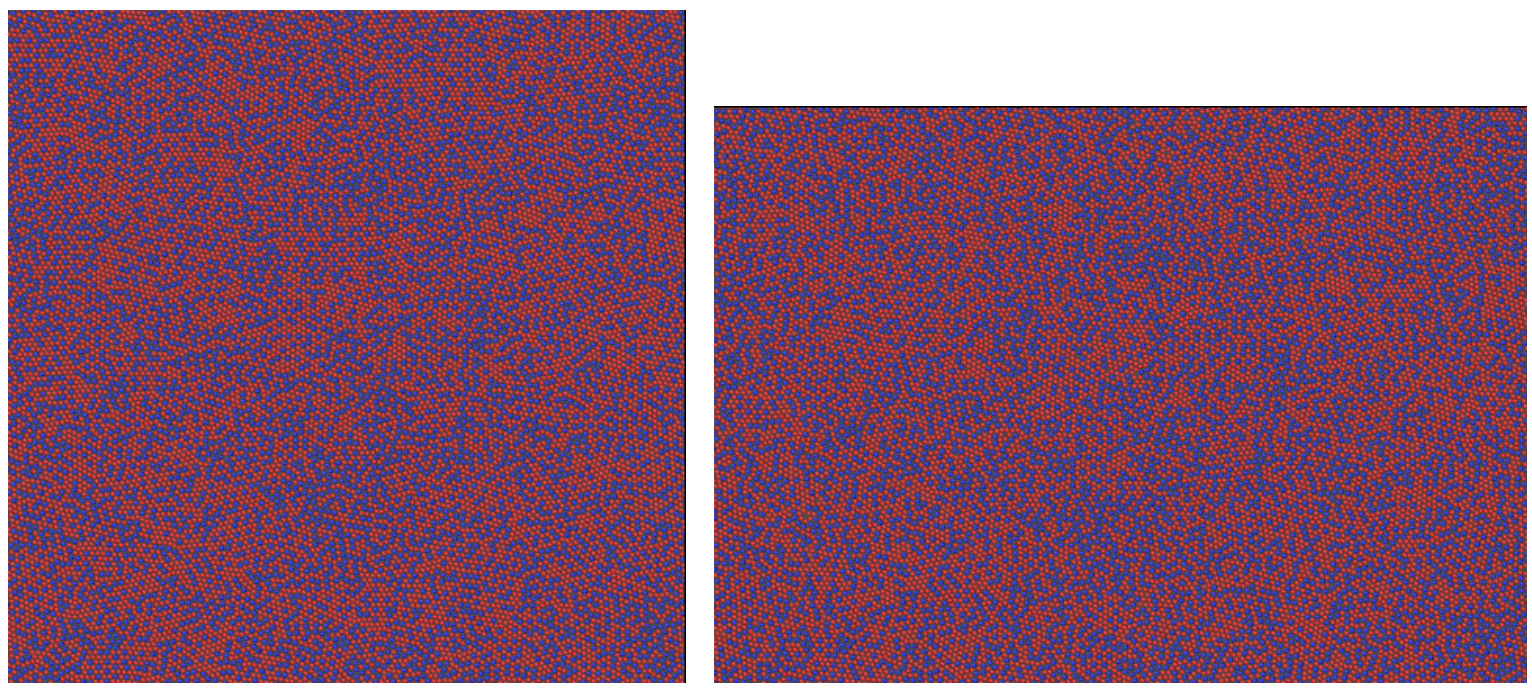

Figure 5.1: Specimen dimensions.

Specimen dimensions before (left) and after (right) undergoing uniform tension deformation. Load stress is applied in the $\mathrm{x}$ direction, forcing the system to contract in the y direction to alleviate the stress.

Load is applied in the $\mathrm{x}$ direction, forcing the system to expand/contract in the y direction to alleviate the stress. In other words, the specimens were constrained to only be able to expand or contract freely in the $\mathrm{y}$ direction and not in the $\mathrm{x}$ direction, hence, the resulting specimen looks like a rectangle as seen in figure 5.1. These figures represent the initial state of the specimen and the final state of the specimen after undergoing a deformation of $\epsilon=20 \%$.

In chapter 4 , the methodology for the creation of the true specimen and the Wang tiling specimen was presented. After several iterations were performed, and the final proposed methodology was established, eight different true specimens were created. In order to ensure that they were all representative of the same metallic glass, their mechanical properties (bulk and Young modulus) were calculated; there was slight variation amongst the specimens however they are representative of a $\mathrm{Cu}-\mathrm{Zr}$ metallic glass (refer to section 4.1 for more details on the calculation of the Young modulus). A similar procedure is used for determining the bulk modulus, the difference is in 


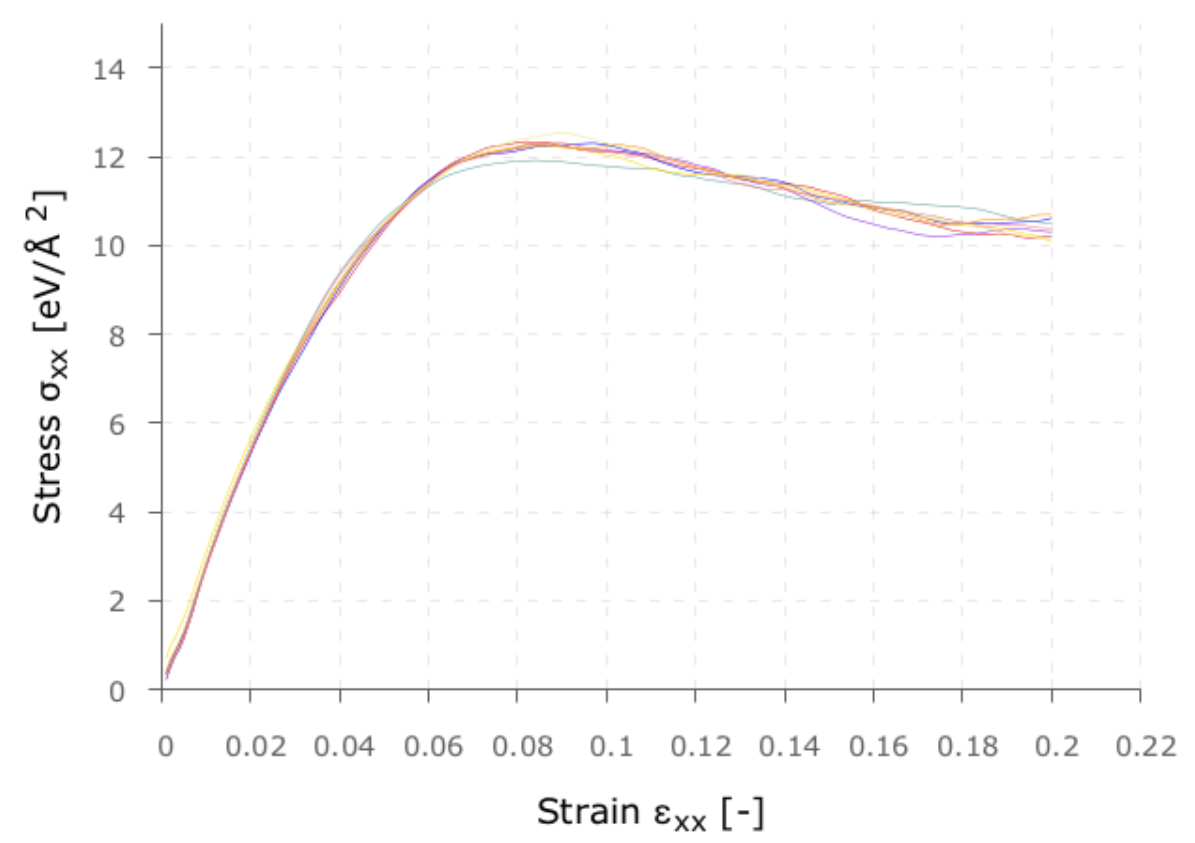

Figure 5.2: Stress-strain curve comparison for eight different true specimens.

The Young modulus for the true specimens is $387.89 \pm 10.92 \mathrm{eV} / \AA^{2}$. Determining the modulus's value for each specimen was described in figure 4.4.

There is an average $0.12 \mathrm{eV} / \AA^{2}$ Root-Mean-Square (RMS) difference between the curves.

the graph that is used for it's estimation; the bulk modulus is determined from a pressure-volume graph.

The macroscopic observations performed in this section are based on the stressstrain curve. The stress-strain curve presents two points of particular interest for the comparison of specimens: the elastic limit and the maximum strength. The elastic limit refers to the limit beyond which the material will not go back to it's original position (defined as the point where the inelastic deformation reaches $0.2 \%$ ); this point is considered to be the intersection between the original graph and a linear graph offset by $0.2 \%$ from the origin where E is the Young modulus of the graph, an example on how this point is determined can be seen in figure 5.3. The maximum strength on the other hand is the ultimate stress a material can withstand, in the 
stress-strain graph this point is represented as the highest peak in the curve and is the main point used for comparison between specimens as it is easier to visualize.

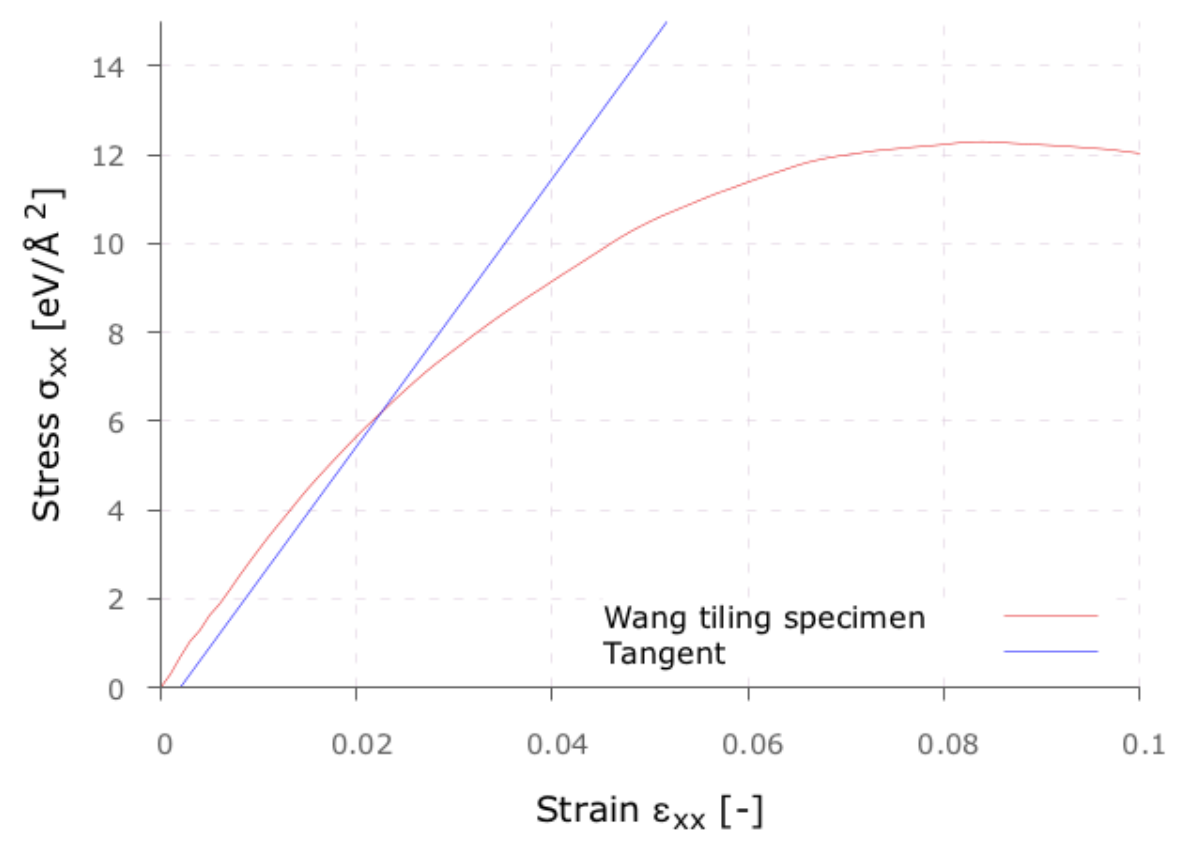

Figure 5.3: Estimation of the elastic limit from the stress-strain curve.

A line is constructed parallel to the initial portion of the stress-strain curve but offset by $0.2 \%$ from the origin. The elastic limit is the point where the constructed line intersects with the stress-strain curve as seen in the figure (approximately $2.2 \%$ strain).

One of the concerns with creating different true specimens is whether their initial configuration plays a significant role during deformation. Even though they all have similar moduli, this does not mean they will undergo inelastic and plastic deformation in the same manner. Figure 5.2 shows the stress-strain curve of the eight true specimens; they were all submitted to an $\dot{\varepsilon}$ of $0.011 /$ picosecond. It can be observed that all specimens experience the similar plastic deformation and that the main differences amongst them is in their maximum strength (the maximum strength is the maximum ordinate in the graph). The difference between specimens is quantified by determining the average Mean Square and Root-Mean-Square distance between 
the curves, specifics on how these values are determined is presented shortly in the chapter.

A similar analysis then needed to be performed on the Wang tiling specimen. In section 4.2, the methodology for the creation of a Wang tiling specimen was established. The tiling assembly is conditioned by corner code compatibilities, therefore, different tiling assemblies can result from the same set of prototiles. Some questions of interest are:

1. Is there a difference between tilings assembled using the same tile set?

2. If the same reference configuration is used to create a new tile set, is there a difference between tilings?

3. Is there a difference in deformation between tilings assembled from different tile sets that originate from different true specimens?

These questions have several follow up questions associated with them. If tilings assembled from the same tile set (use of the same samples, prototiles and Wang tiles) result in differences, is it because the system has not yet reached an energy minimum, or does system deformation depend on the atomic configuration history? If the history of the system plays a role, this same dependence would then be applicable if the tilings assembled from different tile sets also exhibited differences, where these tile sets could be formed from the same true specimen or not.

In order to quantify the differences between specimens, the Root-Mean-Square (RMS) difference between the curves is determined. To determine the RMS difference between two specimen curves (where $a$ and $b$ are different specimens), first we need to find the square displacement for all strain values. Once they are determined, they are averaged, resulting in the Mean-Square (MS) difference. The RMS difference is the 
square root of the MS difference and is measured in $\mathrm{eV} / \AA^{2}$ (see equation 5.2 , where $\mathrm{n}$ is the number of steps it takes to get from $\epsilon_{x x}=0 \%$ to $\epsilon_{x x}=20 \%$ ).

$$
R M S=\sqrt{M S}=\sqrt{\frac{\sum_{\epsilon_{x x}=0 \%}^{\epsilon_{x x}=20 \%}\left(\sigma_{a}-\sigma_{b}\right)^{2}}{n}}
$$

The first approach is looking at deformation for different tilings assembled from the same tile set. Two tilings were assembled using the same tile set. There were no differences in the prototiles used, but the tilings are different due to different random choices in the assembly. There is no significant macroscopic difference to take into account during the deformation of these tiled specimens, as can be seen in figure 5.4; differences between both tilings start to present themselves close to the maximum strength.

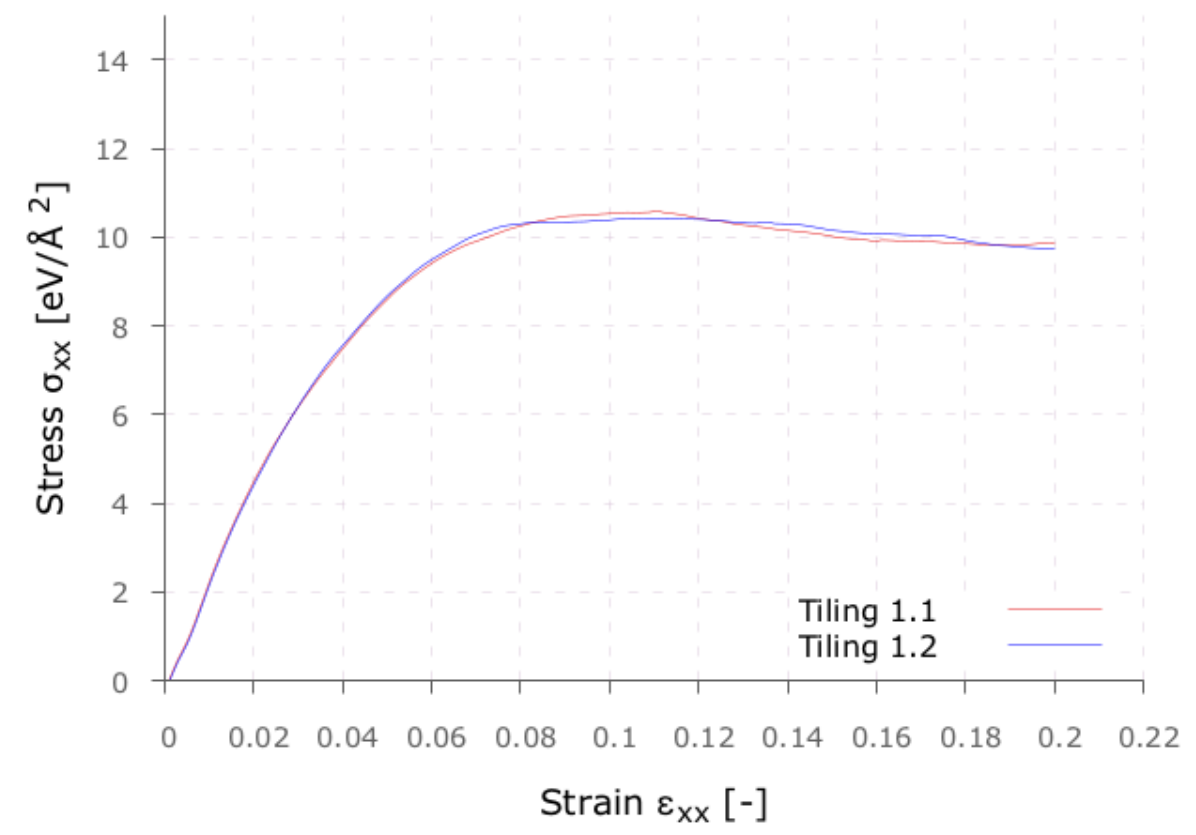

Figure 5.4: Stress-strain curve comparison for two different tilings (same tile set).

The tilings are assembled from the same tile set (same samples, prototiles, and Wang tiles). There is a $0.101 \mathrm{eV} / \AA^{2}$ Root-Mean-Square (RMS) difference between the curves. 
The second approach is to look for differences between tilings that were assembled using different tile sets that were constructed from the same true specimen. In this case, the same true specimen was used, eight samples were extracted from it and the different tile sets arise from varying the samples used for the creation of prototiles and Wang tiles. From figure 5.5, a difference between the three curves is easily observable. Some tilings are more resistant to stress deformation than others.

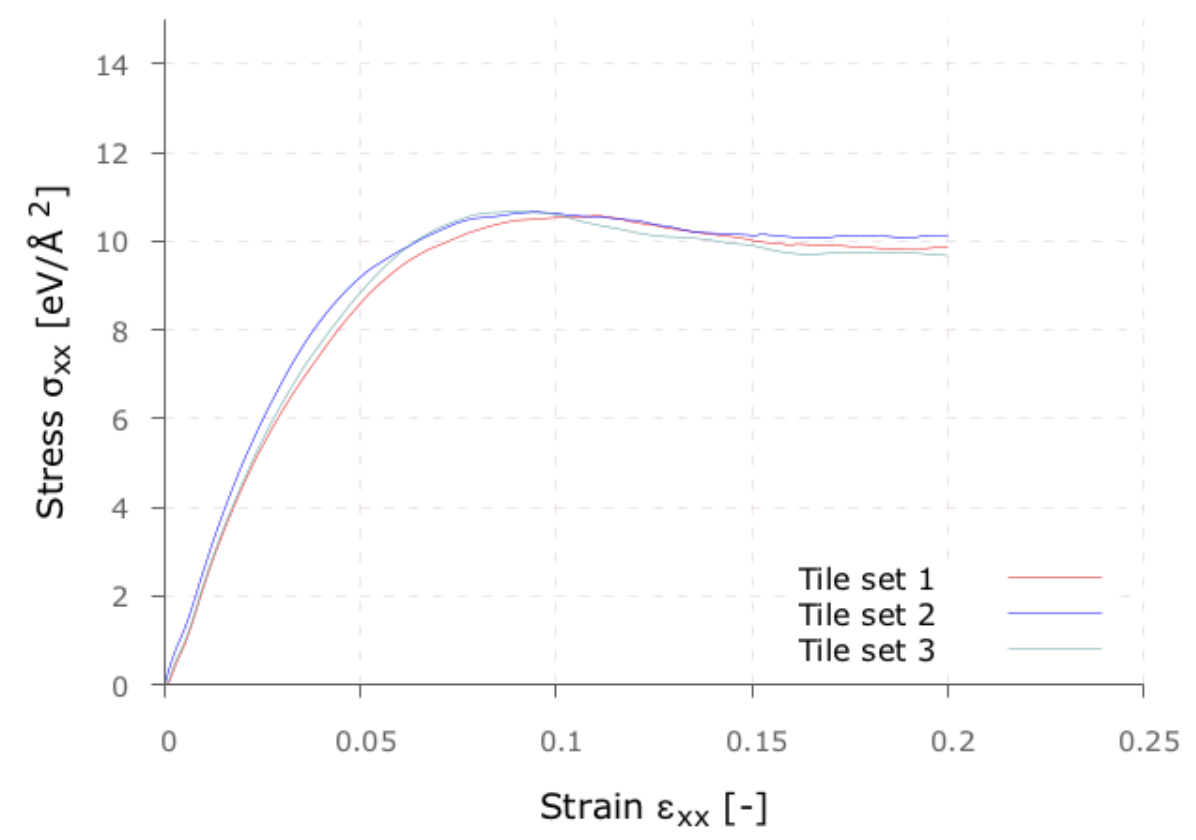

Figure 5.5: Stress-strain curve comparison for three different tilings (different samples).

The samples are assembled from different samples, samples are extracted from the same specimen. There is an average $0.21 \mathrm{eV} / \AA^{2}$ RMS difference between all the curves.

The third approach is to look for differences between tilings that are formed from different true specimens. The reference specimen can be any one of the eight true specimens that were created in section 4.1. Eight different tilings were assembled in total, one for each true specimen, and they were all submitted to uniaxial tension as in the prior approaches. Figure 5.6 shows the results from the deformation, while in the 
previous two approaches there were some differences, they could still be considered to be reasonably similar as the difference between tilings was less than an average 0.21 $\mathrm{eV} / \AA^{2}$ RMS difference, however, there is great variance when looking at tilings from different true specimens, average $0.49 \mathrm{eV} / \AA^{2}$ RMS difference.

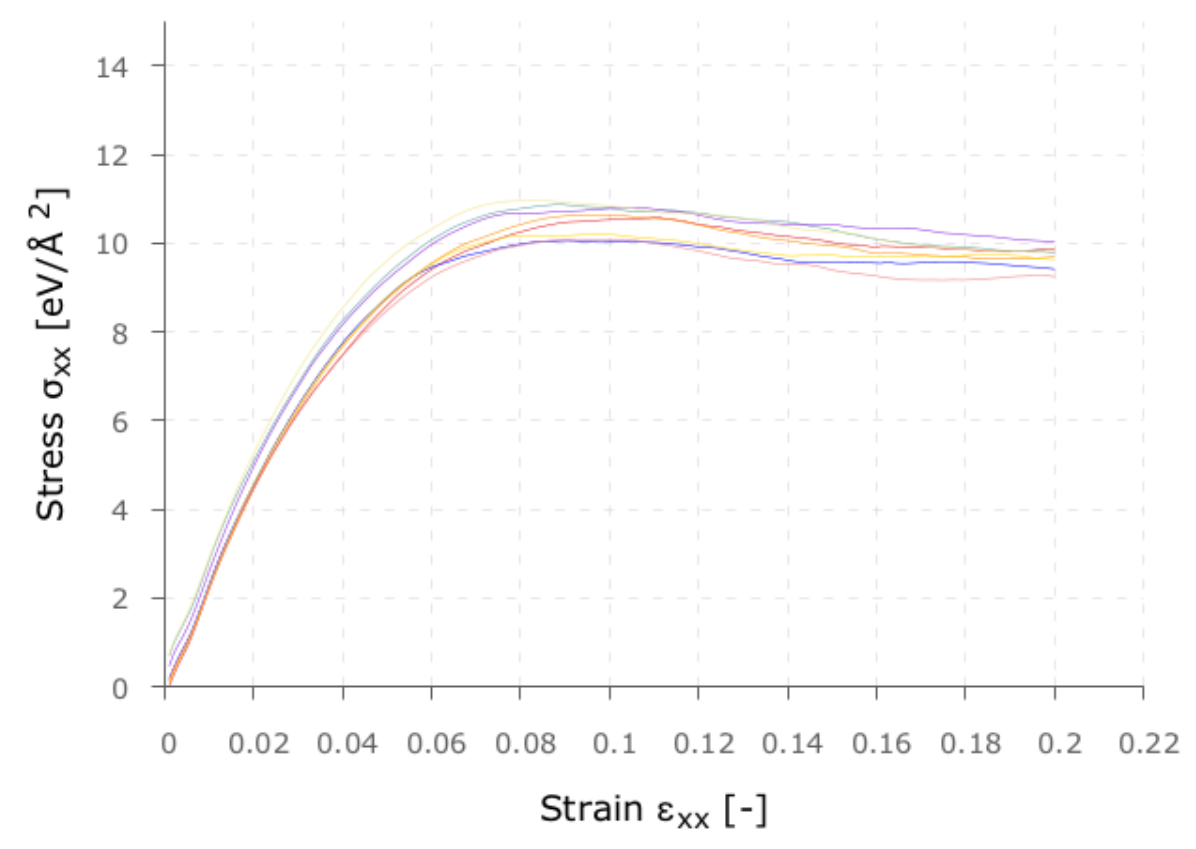

Figure 5.6: Stress-strain curve comparison for eight different tilings with samples from different specimens.

Samples are extracted from different true specimens, there is one tiling for each true specimen created; therefore, all tilings have different prototiles, Wang tiles, and tile sets. There is an average $0.49 \mathrm{eV} / \AA^{2}$ RMS difference between the tilings.

The observations of figures 5.4, 5.5 and 5.6 demonstrate that:

1. Tilings that are assembled using the same tile sets present small differences ( $\left.\mathrm{RMS}=0.101 \mathrm{eV} / \AA^{2}\right)$ when submitted to uniform tension deformation.

In this approach, the same 16 Wang tiles (that form a tile set) are assembled two consecutive times and the variations in the tilings are a result from the stochastic tiling of the lattice. 
2. Tilings assembled from different tile sets, but from the true specimen, present more visible differences $\left(\mathrm{RMS}=0.21 \mathrm{eV} / \AA^{2}\right)$.

3. Using different true specimens leads to differences in Wang tiling specimens that result in big differences. Some specimens are more susceptible to plastic deformation than others $\left(\mathrm{RMS}=0.49 \mathrm{eV} / \AA^{2}\right)$.

These observations might result from differences in the atomic configuration of the system, the way they respond to stress and the formation of shear bands in the system. This microscopic observations will be looked at in the following section, section 5.2.

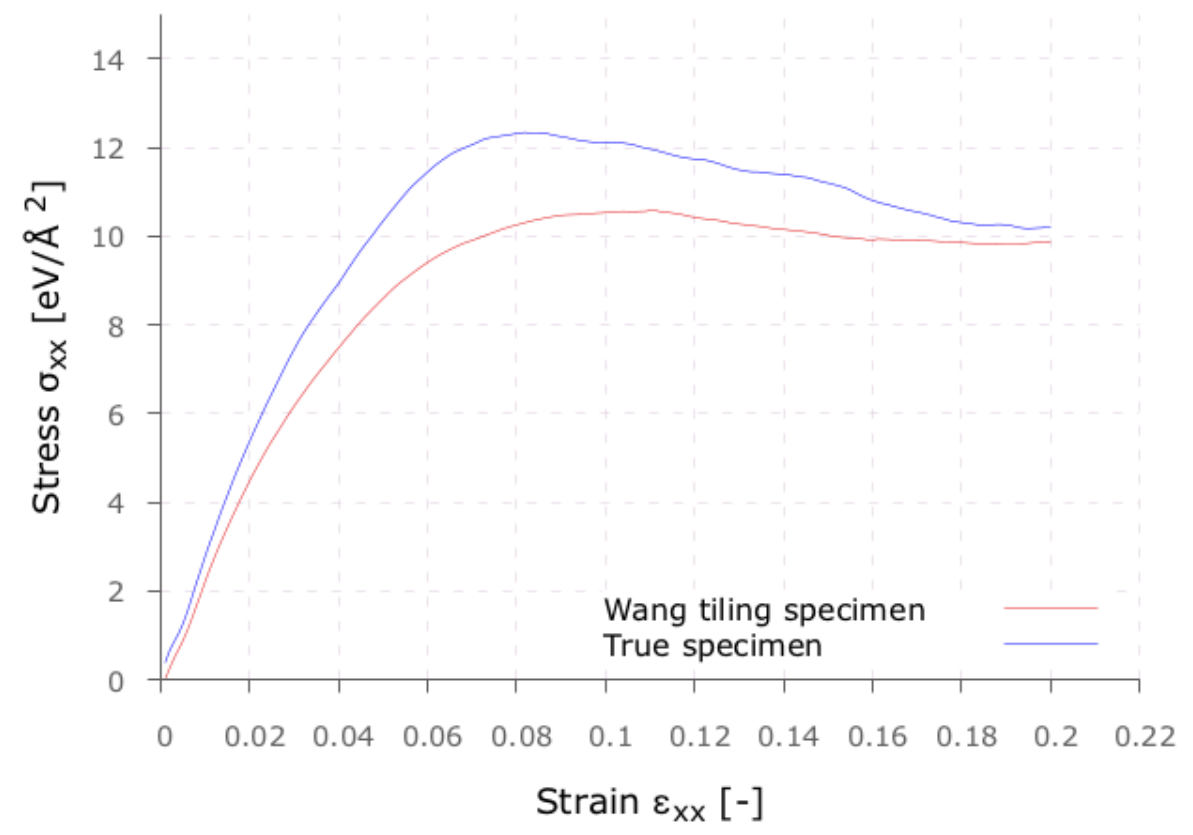

Figure 5.7: Stress-strain curve comparison for a true specimen and a Wang tiling specimen.

A true specimen and a Wang tiling specimen considered to be the average of their respective population was used for this comparison. Both specimens were submitted to the same uniform tension deformation. There is a RMS difference between both specimens of $1.35 \mathrm{eV} / \stackrel{\circ}{ }^{2}$.

Until now, the deformation of both specimens has been presented independently. 
One of the objectives of this research is to determine whether there is a difference between a randomly generated specimen (true specimen) and a Wang tiling specimen. There is no significant variation in specimens, figure 5.2, therefore any specimen can be used in the comparison. However, there is variance in the Wang tiling specimens as seen in figure 5.6, therefore an average on the tiling specimens will be used for the comparison. An average specimen from both populations was selected for the comparison, this comparison can be seen in figure 5.7.

There is a great difference between both specimens. The Wang tiling specimen suffers inelastic deformation at much smaller stresses than the original specimen, figure 5.7. At low strain, both materials behave in a linearly elastic manner. As the strain increases, the response becomes nonlinear and the material begins to deform plastically. Both specimens present very different maximum strengths, the true is more resistant to deform at larger stress values. There the Root-Mean-Square distance between both specimens is of $1.35 \mathrm{eV} / \AA^{2}$, where the Wang tiling specimen is weaker than the true specimen.

The differences in the curves between both specimens, leads to the conclusion that the Wang tiling specimen is not representative of the $\mathrm{Cu}-\mathrm{Zr}$ metallic glass or that it is yielding a weaker variant that undergoes plastic deformation at lower stresses. One possible explanation for this difference is their atomic configuration, an idea that is explored in the next section.

\subsection{Microscopic observations}

In addition to the observation of stress and strain at the macroscopic level, the average features of the population might be of interest, specially if two-state shear transformation zones occur or if there are local defects present in the specimens, e.g. atomic 
bond deficiency defects.

Atomic bond deficiency (BD) defects are commonly found in amorphous solids and are geometrically similar to crystal vacancies [61]. BD is a deviation from the coordination number of the ideal atomic structures caused by chemical and topological short-range ordering. Zhu et al. [61] concluded that BD defect concentration influences the glass transition phase and the mechanical properties of a glass. In section 5.2 .1 , an analysis of the BD defect concentration of both specimens is presented.

Shear transformation zones $(\mathrm{STZs})$ are small regions where the atomic configuration makes it susceptible to inelastic rearrangements in response to shear stresses [17]. The shear transformation zone model used here is similar to versions presented by Argon, Spaepen and others for the description of creep in metallic alloys [16,20,24]. An analysis on the presence of shear transformation zones in both specimens is presented in section 5.2.2.

The major difference between the BD model and the STZ model is that the STZ model considers that a collective movement of atoms inside the zone of applied stress will give rise to a uniform shear strain; the BD model considers shear will occur due to the uneven displacement of atoms involving multiple bond deficiencies. Both models would prove the formation of shear bands in the specimens.

\subsubsection{Atomic Bond Deficiency defects (BD defects)}

Defects are microstructural features that give a material its characteristic properties. When working with crystalline solids, these defects can be: surfaces, grain boundaries,

dislocations or point defects $[32,62]$. In amorphous solids, atomic bond deficiency defects (BD defects) are considered to be one of the most characteristic structural differences [63].

BD defects are regions with low packing efficiency. In metallic glasses, these defects 
are sometimes considered extra volumes [64], that can be conceived as voids or holes in a structure, figure 5.8 [63]. The presence of defects in amorphous metals play a controlling role in atomic transport processes such as diffusion, structural relaxation and plastic flow [65].
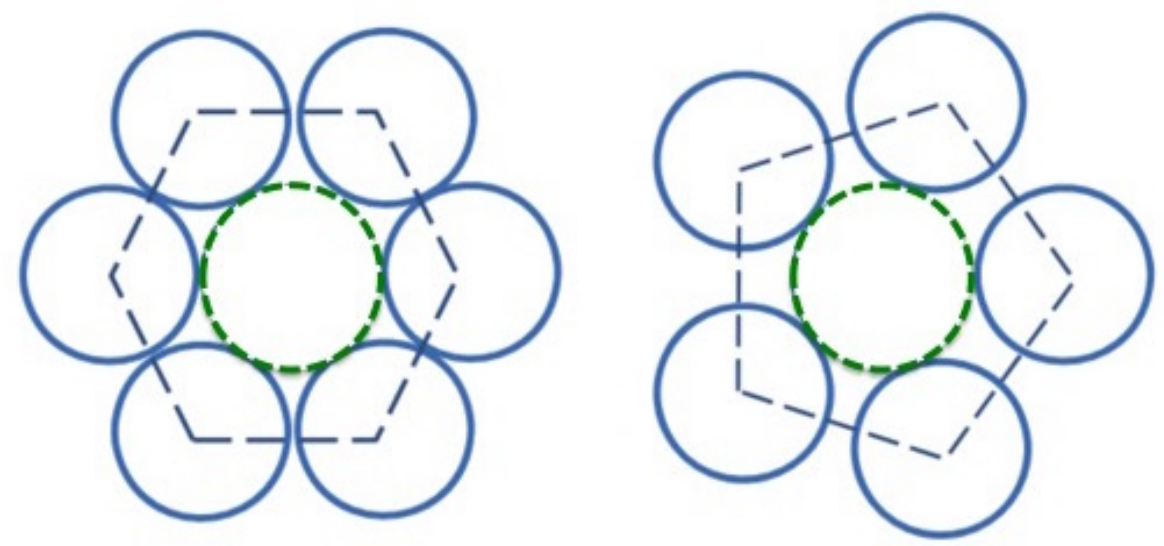

Figure 5.8: Vacancy defect and atomic Bond Deficiency (BD) defect comparison. Visual representation of a vacancy defect in a crystalline structure (left) and a single BD defect in an amorphous structure (right). The BD defect is a short-range order defect. The centre particle depicted in both images is missing the structures, creating a 6-atom or a 5-atom ring around the location. Reproduced from [63].

BD defect formulation is based on two fundamental notions: (i) atomic movement is restricted and (ii) only short-range order constrains the formation of this structural defect. BD defects are formed from thermal fluctuations or geometrical restrictions, where chemical disordering and bond deficiencies are examples for each respectively.

$\mathrm{BD}$ defects are considered diffuse carriers (motion diffusion carriers) that influence the mechanical properties of amorphous materials. Because of the small free volume of the $\mathrm{BD}$, adjacent atoms are dependent on its interaction for an effective displacement to take place. This process can be viewed as the relocation of $\mathrm{BD}$ regions and is dependent on the surrounding atomic interactions [63].

In this research, there is interest in studying BD defect based configurations as 
shear flow carriers. The cooperative and uneven (even opposite) movement of multiple bond deficiencies can lead to shear flow [63].

In the prior section, the deformation of the true specimen and the Wang tile specimen were significantly different $\left(\mathrm{RMS}=1.35 \mathrm{eV} / \AA^{2}\right)$. Considering that a difference in the concentration of $\mathrm{BD}$ defects can be influential in the properties of a structure, the aim of this section is to:

1. Determine if BD defects are present.

2. Determine when BD defects are forming.

3. Determine if BD defect concentration is influential to the deformation of each specimen.

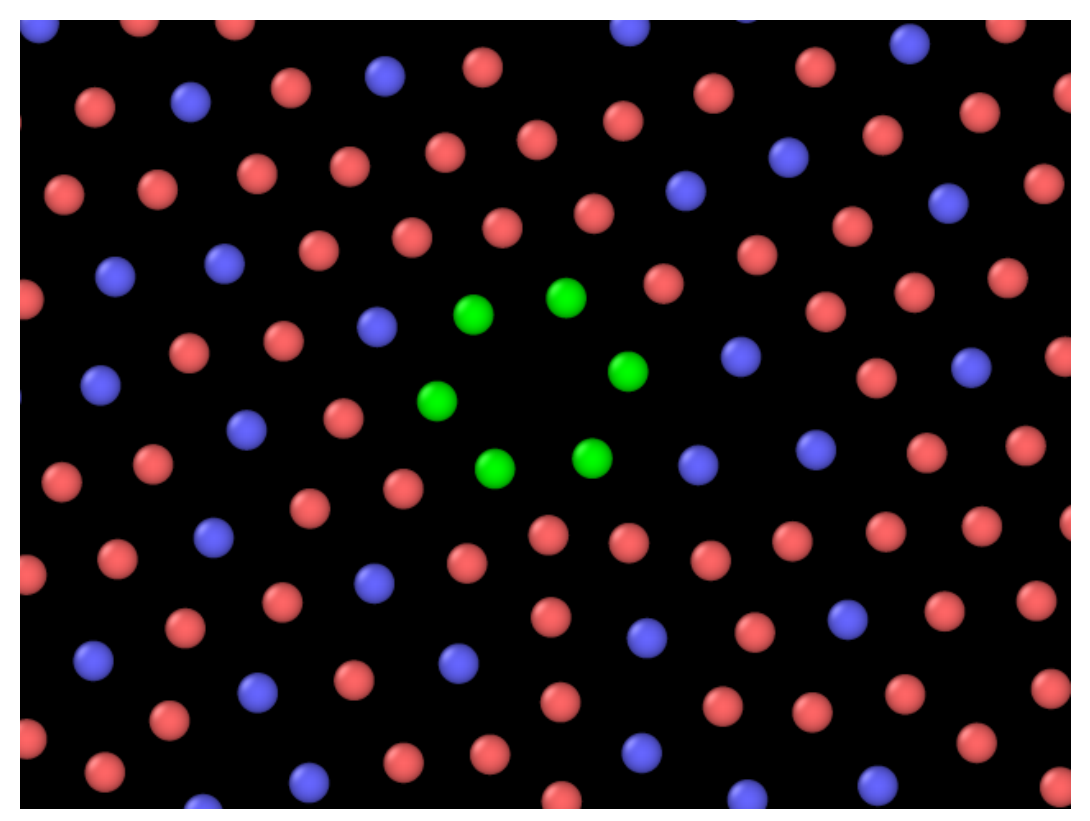

Figure 5.9: Example of a BD defect in a Wang tiling specimen

Atoms encircling the $\mathrm{BD}$ defect are coloured green. Red and blue represent $\mathrm{Cu}$ or $\mathrm{Zr}$ atoms respectively. 
The first thing that needs to be determined is whether BD defects can be found in the true specimen and in the Wang tiling specimen. The reference specimens that were used for the macroscopic comparison between both specimens (used for the development of figure 5.7), were microscopically analyzed to determine the presence of $\mathrm{BD}$ defects at two different timesteps, at the beginning of the deformation $\left(t_{0}\right)$ and when the specimen reached it's maximum strength when subjected to the uniaxial tension deformation $\left(t_{\max }\right)$, this moment was determined from the stress-strain graph of each specimen respectively. Determining the frequency with which these issues presented themselves (number of BD defects as a function of the total number of atoms) allowed us to determine the concentration of $\mathrm{BD}$ defects, $[\mathrm{BD}]$. This value is the number used for comparison at different timesteps.

The existence of BD defects was determined by performing a visual analysis of both specimens (true specimen and Wang tiling specimen). This analysis was performed through the OVITO visualization software. Regions where a visible gap surrounded by 5 or 6 neighbouring atoms were considered BD defects (as seen in figure 5.9).

The true specimen does not present very many BD defects at the beginning of the deformation, however, BD defects are formed during the deformation process, figure 5.10. In contrast, the Wang tiling specimen has a lot of BD defects already present before deformation, figure 5.11. Visually, BD defects appear following a geometry in this specimen (they can be found in straight lines along original tile edges). The presence of these defects can be due to a low density in the corners of the Wang tiles assembled that during minimization yielded these local defects. The BD defects concentration also appears to either remain the same or decrease slightly in the semi-random specimen (Wang tiling specimen), a quantitative analysis needs to be performed for more clarity. 


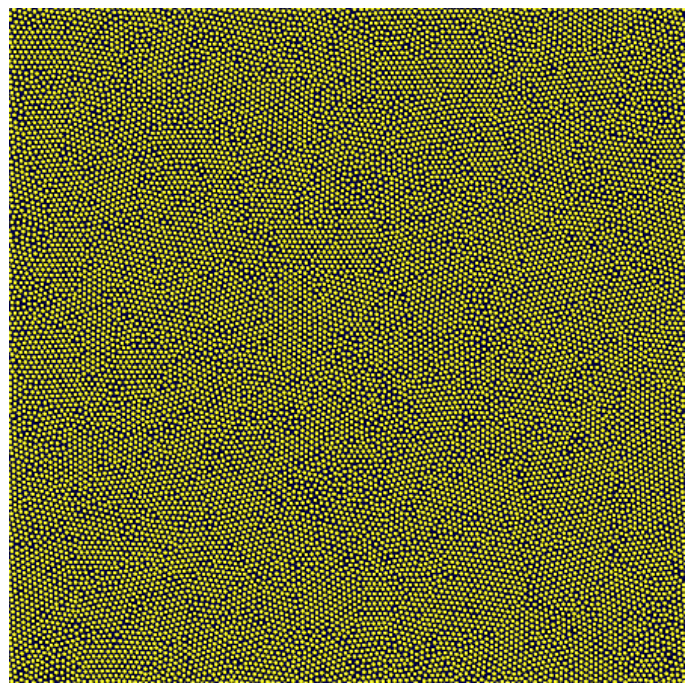

(a) $t_{0},[\mathrm{BD}]=0.017$

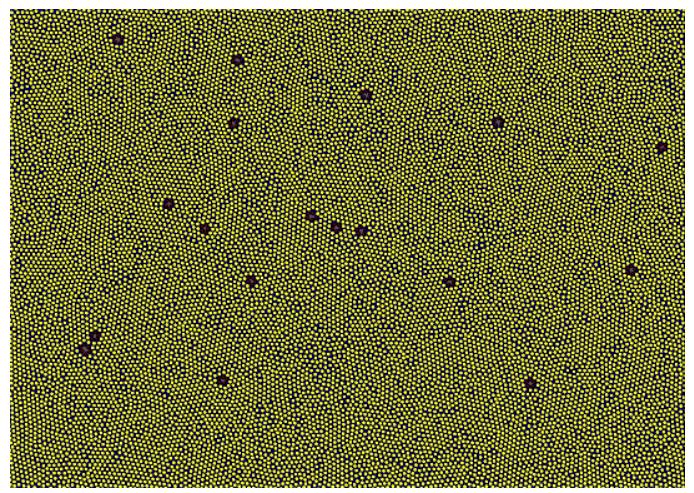

(b) $t_{\max },[\mathrm{BD}]=0.1679$

Figure 5.10: True specimen BD defects.

$\mathrm{BD}$ defects are highlighted in the true specimen for two different timesteps: before undergoing uniaxial tension deformation $\left(t_{0}\right)$ and when reaching the maximum strength $\left(t_{\max }\right)$.

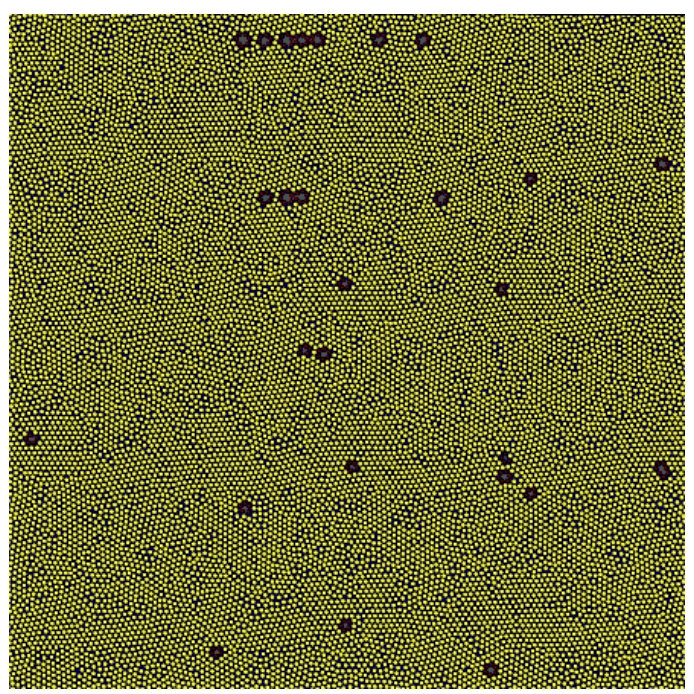

(a) $t_{0},[\mathrm{BD}]=0.1789$

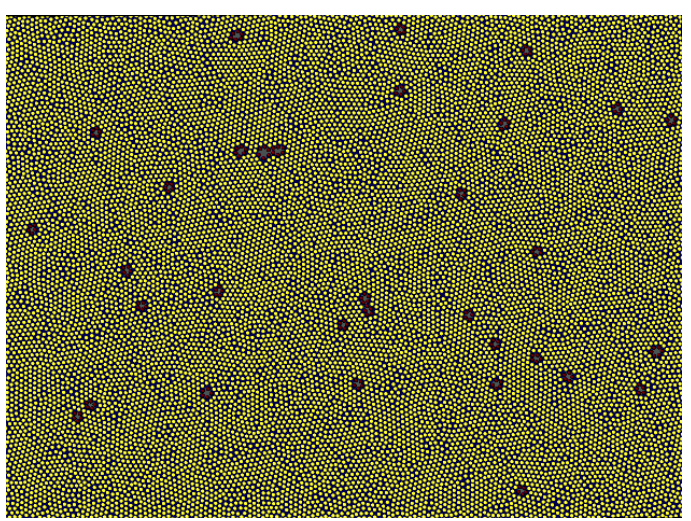

(b) $t_{\max },[\mathrm{BD}]=0.1840$

Figure 5.11: Wang tiling specimen BD defects.

BD defects are highlighted in the Wang tiling specimen for two different timesteps: before undergoing uniaxial tension deformation $\left(t_{0}\right)$ and when reaching the maximum strength $\left(t_{\max }\right)$. 
An analysis on the nearest neighbours per particle was performed in order to determine the location of $\mathrm{BD}$ defects. It was found that in regions where BD defects are present, there is a difference in the number of neighbours the surrounding atoms have. Instead of having 30 atoms as nearest neighbours, they can have more or less depending on how far they have been displaced by the void.

The figures presented are proof that BD defects are present in both specimens. It can also be observed that there is a difference in BD defect concentration throughout the process, BD defects are being formed throughout the deformation. The formation of one of these defects is presented in figure 5.12; for visualization purposes, atoms that form part of the defect are coloured green, these atoms can be either $\mathrm{Cu}$ or $\mathrm{Zr}$ particles.
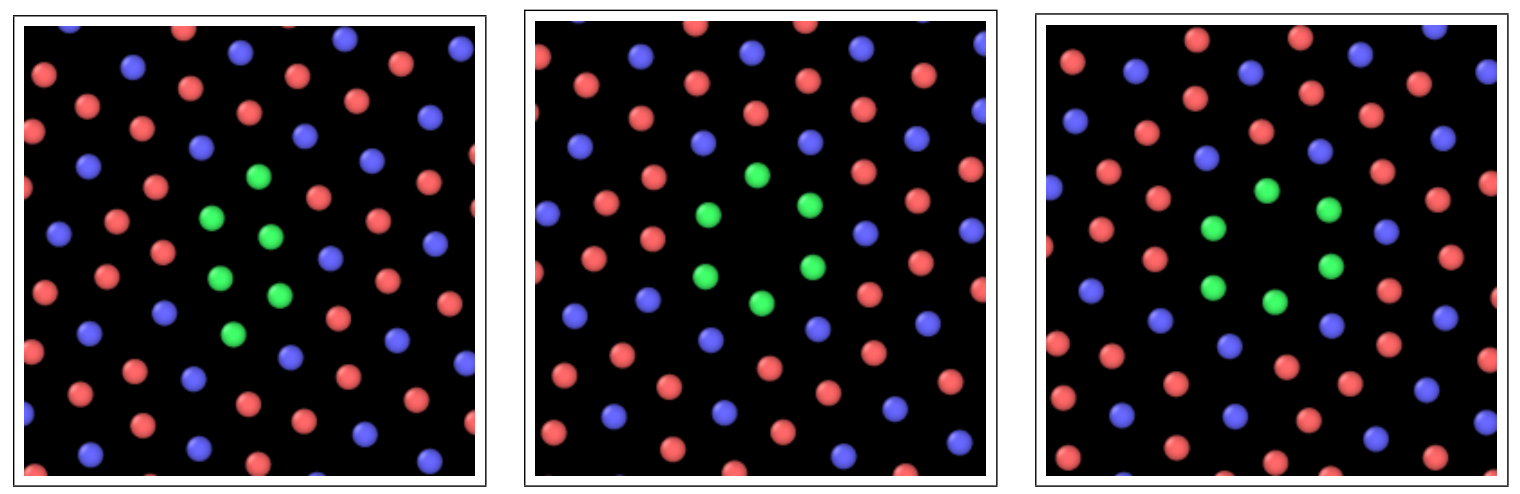

Figure 5.12: BD defect formation

Formation of a BD defect during uniform tension deformation for the true specimen. The images represent three different timesteps: $t=t_{0}$ (left) $t=t_{\text {max }}$ (centre) and $t=t_{\text {final }}$ (right).

So far, it has been proven that some specimens present BD defects either from their initial configuration (as seen in the Wang tiling specimens) or from their formation throughout the deformation process (as seen in figure 5.12).

The BD defect concentration influence on a system's properties was presented by Zhu et al. [63], in their work a statistical thermodynamic analysis of binary systems 


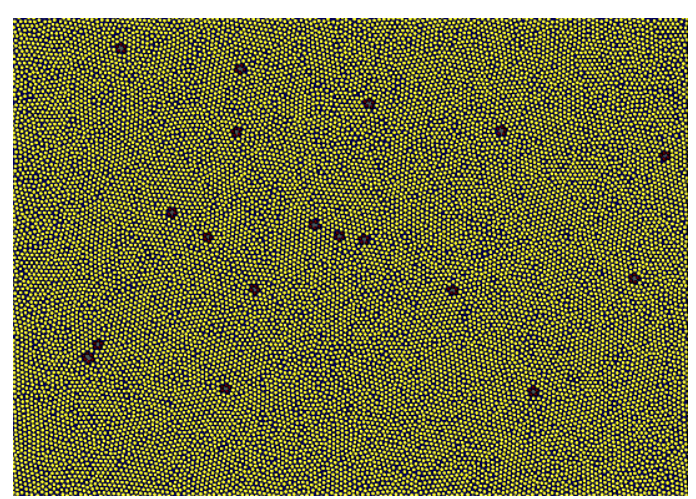

(a) $[\mathrm{BD}]=0.1679$

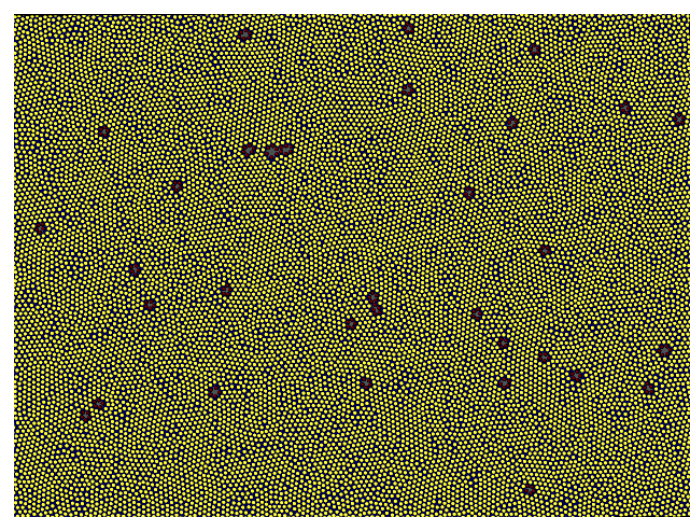

(b) $[\mathrm{BD}]=0.1840$

Figure 5.13: BD defects at the maximum strength, $t_{\max }$.

$\mathrm{BD}$ defects are highlighted. The left image represents the true specimen and the right the Wang tiling specimen. There is a higher concentration of BD defects in the tiling than in the true specimen.

showed that systems with higher BD defect concentrations were more susceptible to plastic flow of their particles. The distinct difference between the maximum strength of the specimens can be attributed to a difference in their BD defect concentrations. One explanation for the higher maximum strength exhibited by the true specimen can be the lower concentration of BD defects at that timestep, as seen in figure 5.13.

The difference in stress-strain curves between specimens might be influenced by the formation of BD defects in the specimens. According to Zhu et al., the appearance of these defects condition the transportation of particles inside a system [63]. Therefore, specimens that present these defects (Wang tiling specimen) might present atom movement that is influenced by the presence of the defects; the defect will move as a unit throughout the volume. The mobility of the clusters might lead to lower maximum strengths than in systems without them (e.g. the true specimen). 


\subsubsection{Local atomic Mises strain, $\eta_{i}^{\text {Mises }}$, observation}

The local atomic Mises strain $\left(\eta_{i}^{\text {Mises }}\right)$ permits quantifying the plastic deformation of a system at an atomic level. Its calculation requires two atomic configurations: the current configuration (configuration of particular interest) and a reference configuration to which atom displacement is to be compared (configuration at $t=t_{0}$ is commonly used).

Determining $\eta_{i}^{\text {Mises }}$ consists of three general steps, that were adapted to fit a two-dimensional system:

1. Jacobian calculation.

2. Calculation of local Lagrangian strain matrix.

3. $\eta_{i}^{\text {Mises }}$ calculation.

The first step is to determine the local transformation matrix, also known as the Jacobian, $\boldsymbol{J}_{\boldsymbol{i}}$ that best maps the vector separations $\boldsymbol{d}$ between two atoms, atom $i$ and atom $j$, equation 5.3; where the superscript 0 means the reference configuration (considered to be the configuration at $t=t_{0}$ for this section of the microscopic analysis).

$$
\begin{gathered}
\left\{\boldsymbol{d}_{\boldsymbol{j} i}^{\mathbf{0}}\right\} \rightarrow\left\{\boldsymbol{d}_{\boldsymbol{j i}}\right\}, \forall j \in N_{i}^{0} \\
\boldsymbol{d}_{\boldsymbol{j i}}=\boldsymbol{d}_{\boldsymbol{j} \boldsymbol{i}}^{\mathbf{0}} \boldsymbol{J}_{\boldsymbol{i}}
\end{gathered}
$$

In equation 5.3, atoms $i$ and $j$ are considered to be nearest neighbours, and $N_{i}^{0}$ is the total number of nearest neighbours that atom $i$ has at $t_{0}$. The number of neighbours taken into consideration is determined through a sampling radius $\left(d_{c u t}\right)$, which in this case was chosen to be the cutoff radius stated in the EAM potential [35]. 
The Jacobian is determined through equation 5.5 using a least squares fit process. Once the Jacobian is found, the local Lagrangian strain matrix can be computed through equation 5.6, where the matrix components are used for determining $\eta_{i}^{\text {Mises }}$, equation 5.8 .

$$
\begin{gathered}
\sum_{j \in N_{i}^{0}}\left|\boldsymbol{d}_{\boldsymbol{j} \boldsymbol{i}}^{\mathbf{0}} \boldsymbol{J}_{\boldsymbol{i}}-\boldsymbol{d}_{\boldsymbol{j} \boldsymbol{i}}\right|^{2} \rightarrow J_{i}=\left(\sum_{j \in N_{i}^{0}} \boldsymbol{d}_{\boldsymbol{j} \boldsymbol{i}}^{\mathbf{T}} \boldsymbol{d}_{\boldsymbol{j} \boldsymbol{i}}^{\mathbf{0}}\right)^{-1}\left(\sum_{j \in N_{i}^{0}} \boldsymbol{d}_{\boldsymbol{j} \boldsymbol{i}}^{\mathbf{0} T} \boldsymbol{d}_{\boldsymbol{j} \boldsymbol{i}}\right) \\
\boldsymbol{\eta}_{\boldsymbol{i}}=\frac{1}{2}\left(\boldsymbol{J}_{\boldsymbol{i}} \boldsymbol{J}_{\boldsymbol{i}}^{T}-\boldsymbol{I}\right) \\
\boldsymbol{\eta}_{\boldsymbol{i}}=\left(\begin{array}{cc}
\eta_{x x} & \eta_{y x} \\
\eta_{x y} & \eta_{y y}
\end{array}\right) \\
\eta_{i}^{\text {Mises }}=\sqrt{\eta_{x y}^{2}+\frac{\eta_{y y}^{2}+\eta_{x x}^{2}+\left(\eta_{x x}-\eta_{y y}\right)^{2}}{4}}
\end{gathered}
$$

The $\eta_{i}^{\text {Mises }}$ is a good measure of the local inelastic deformation, this value was calculated and then visualized through the visualization program OVITO [34]. Atoms were colour coded according to their $\eta_{i}^{\text {Mises }}$ value.

The $\eta_{i}^{\text {Mises }}$ for both specimens was determined at four timesteps of interest: (i) at $t=t_{1}$, this is the first timestep affected by the deformation parameters; this configuration and all others are compared to the initial configuration at $t=t_{0}$. (ii) At $\epsilon_{x x}=2 \%$. (iii) At $t=t_{\max }$ when each specimen reaches their maximum strength. (iv) And finally at $\epsilon_{x x}=20 \%$ which is the end of the uniaxial tension deformation. The location of these timesteps in the stress strain graph can be seen in figure 5.14. Considering that the Wang tiling specimen presented a higher concentration of 


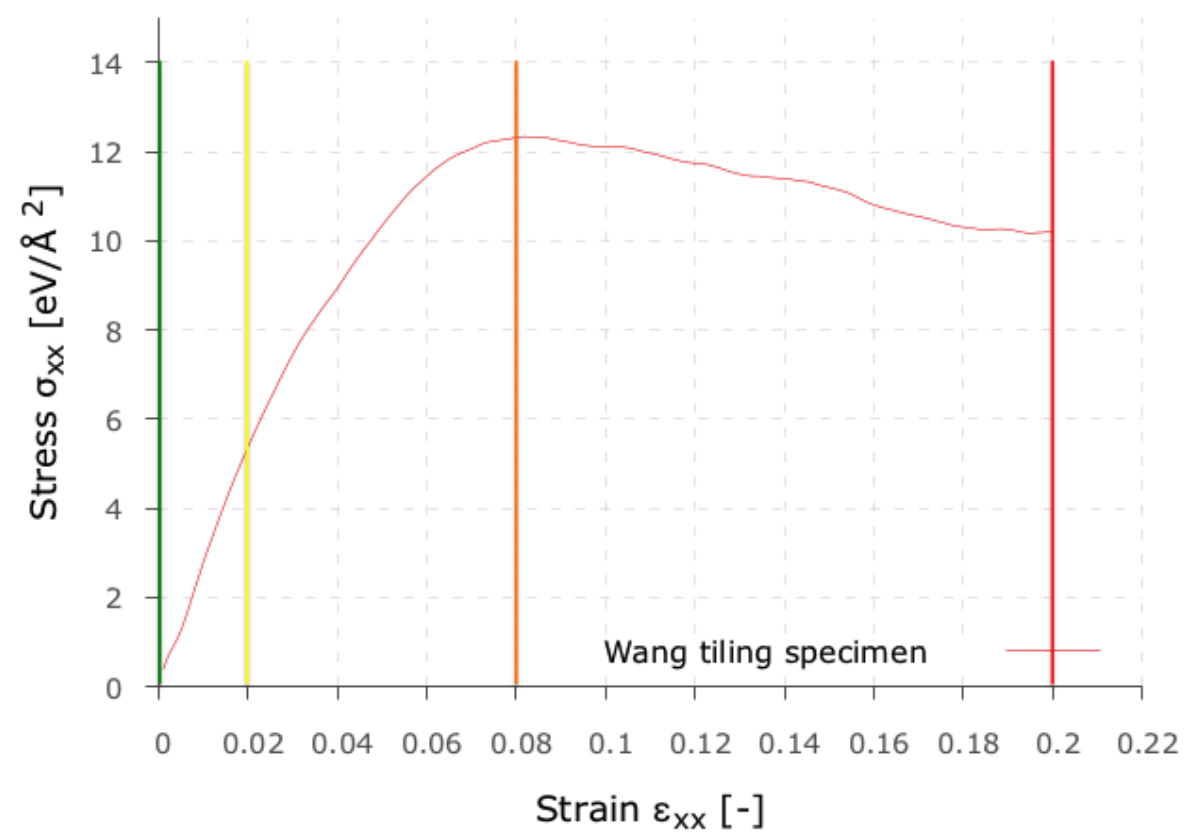

Figure 5.14: Timesteps of interest for visualizing the local atomic Mises strain, $\eta_{i}^{\text {Mises }}$

$\eta_{i}^{\text {Mises }}$ is estimated for four different timesteps: $t=t_{1}$ (green), $\epsilon_{x x}=2 \%$ (yellow), $t=t_{\text {max }}$ (orange), and $\epsilon_{x x}=20 \%$ (red). The second timestep of interest was randomly selected as a strain value between the beginning of the deformation and the maximum strength. The stress-strain graph used to visualize the timesteps is that of the true specimen.

atomic bond deficiency defects (BD defects) than the true specimen, and that BD defects are considered transport enablers that condition atom motion [63], this specimen might exhibit a different $\eta_{i}^{\text {Mises }}$ behaviour.

A comparison of $\eta_{i}^{\text {Mises }}$ at each timestep was performed between specimens. The specimens that are used here for the comparisons were those selected to represent both the true specimen and the Wang tiling specimen in the macroscopic analysis. These specimens are considered the average of their respective populations and present basic features that can be found in all other like specimens.

The intent is to identify the formation of shear transformation zones (STZs) throughout the uniaxial tension deformation of both specimens. Figure 5.15 shows 

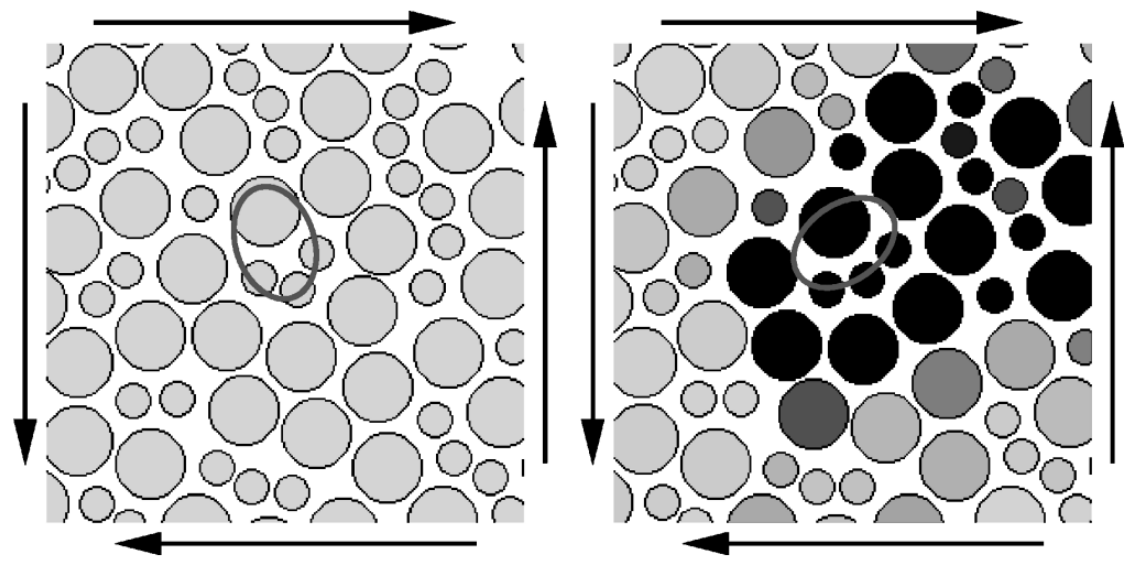

Figure 5.15: Shear Transformation Zone (STZ) before and after deformation. The arrows show the direction of the applied stress and ovals are solely guides for the eye. Reproduced from [24].

an example on what a STZ looks like. A zone is considered to be a STZ when the particles within the region experience an atomic deviation from the original configuration (this deviation is not experienced by the surrounding atoms) [24]. According to Shimizu et al. [17], a shear transformation zone (STZ) has values of $\eta_{i}^{\text {Mises }}>0.3$.

\section{Timestep: $t_{1}$}

The first timestep for each specimen was analyzed. This timestep is the initial reaction of the system to uniaxial tension deformation. The $\eta_{i}^{\text {Mises }}$ for each specimen was determined by the comparison of the current configuration to the initial configuration at $t_{0}$.

The Wang tiling specimens starts to undergo atomic local shear strain $\left(\eta_{i}^{\text {Mises }}\right)$ at a faster rate than the true specimen, as can be seen in figure 5.16. The figure shows the comparison of both specimens after only one timestep being subjected to uniaxial tension deformation. In the true specimen there is a lack of atoms experiencing significant strain, contrary to the semi-random specimen (Wang tilings specimen). It can be expected that as both specimens continue undergoing deformation, $\eta_{i}^{\text {Mises }}$ 


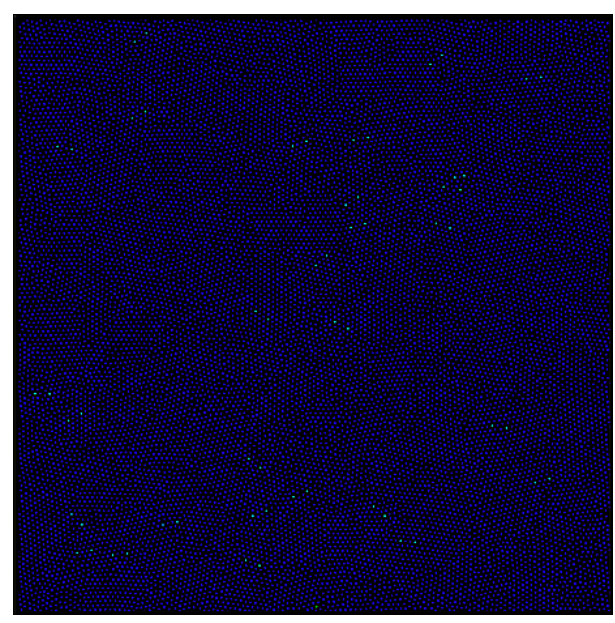

(a) True specimen

$\eta_{i}^{\text {Mises }}$ max $=0.09$

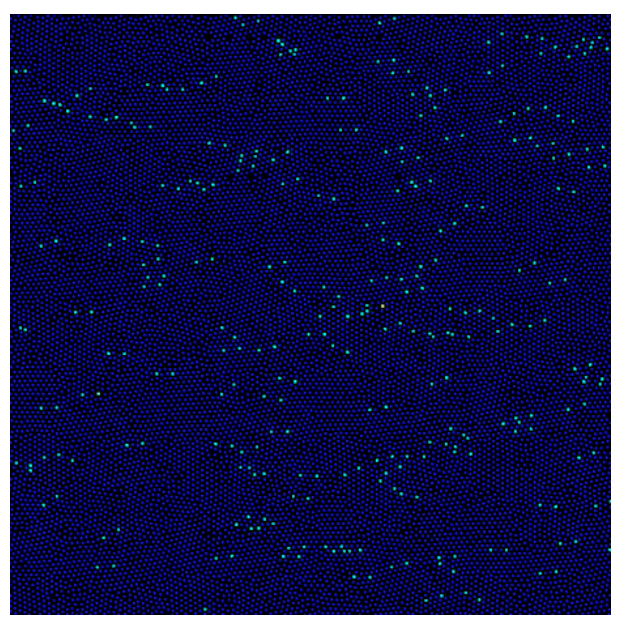

0.18

0.16

0.14

0.12

0.1

0.08

0.06

0.04

0.02

Figure 5.16: Intensity plots of $\eta_{i}^{\text {Mises }}$ for $t_{1}$. Atoms are coloured according to their $\eta_{i}^{\text {Mises }}$ value, ranging from $0.02-0.2$

propagates in the vicinity of the already affected values.

Timestep: $t$ when $\epsilon_{x x}=2 \%$

The $\eta_{i}^{\text {Mises }}$ values for both specimen when $\epsilon_{x x}=2 \%$ were compared.

The intent was to determine whether shear transformation zones were presenting themselves at this timestep. The results of the $\eta_{i}^{\text {Mises }}$ analysis can be seen in figure 5.17 .

In figure 5.16, it was assumed that the coloured points on the Wang tiling specimen would then propagate to form a shear transformation zone (STZ). Through figure 5.17 , it can be inferred that the strain got evenly distributed throughout the entire specimen rather than create large localized shear bands. There is no clear distinction on the formation of STZs, however, a closer look at the regions with the higher $\eta_{i}^{\text {Mises }}$ values at earlier timesteps can clarify whether STZs were initially formed and propagated throughout the system. 


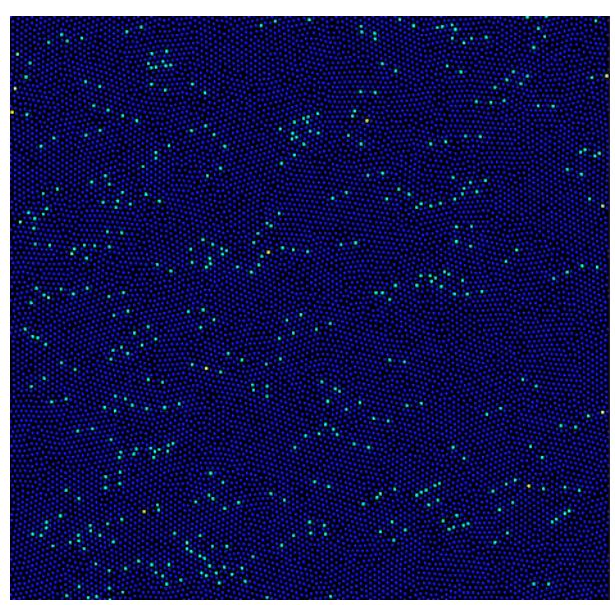

(a) True specimen $\eta_{i}^{\text {Mises }}{ }_{\max }=0.076$

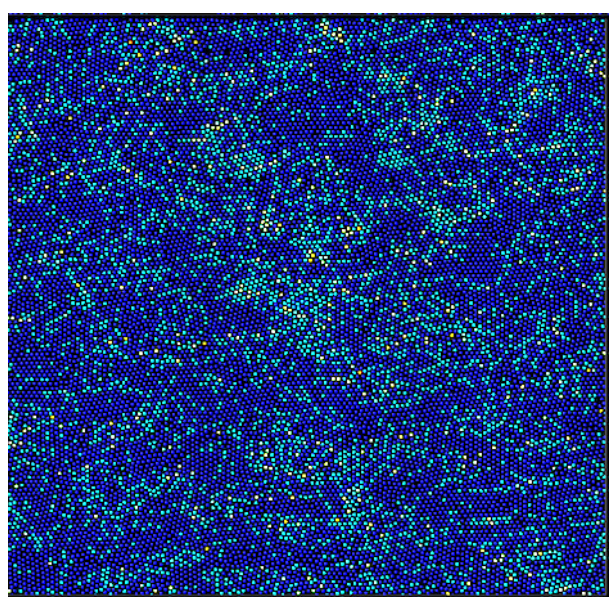

0.2

0.18

0.16

0.14

0.12

0.1

0.08

0.06

0.04

0.02

Figure 5.17: Intensity plots of $\eta_{i}^{\text {Mises }}$ for $\epsilon_{x x}=2 \%$. Atoms are coloured according to their $\eta_{i}^{\text {Mises }}$ value, ranging from $0.02-0.2$

A detailed look at earlier timesteps reveals that some of the initial affected atoms from 5.16 did not lead to STZs as $\eta_{i}^{\text {Mises }}<0.3$ [17]. A closeup view of these zones and their aspect reveals that atoms present high $\eta_{i}^{\text {Mises }}$ values but this does not mean there is a significant atomic rearrangement taking place within the affect zones, as can be seen in figures 5.18 and 5.19 .

A correlation can be implied between the differences in the shear-strain curves of both specimens, and the $\eta_{i}^{\text {Mises }}$ concentration in both specimens. Figure 5.17 shows a large difference in the concentration between specimens. The true specimen does not have as many atoms experiencing $\eta_{i}^{\text {Mises }}$, this might be related to the higher maximum strength that this specimen presents when compared to the Wang tiling specimen.

\section{Timestep: $t_{\max }$}

As the deformation continues, more stress is applied to the system where both specimens show an even distribution of the $\eta_{i}^{\text {Mises }}$ in their volume, as seen in figure 


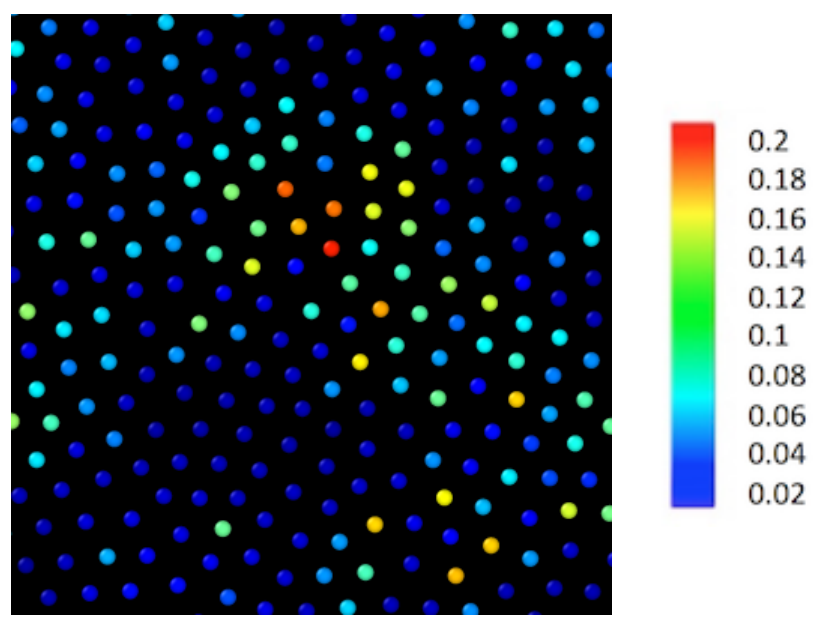

Figure 5.18: High $\eta_{i}^{\text {Mises }}$ value zone in the Wang tiling specimen. Atoms are coloured according to their $\eta_{i}^{\text {Mises }}$ value, ranging from 0.02 to 0.2 .
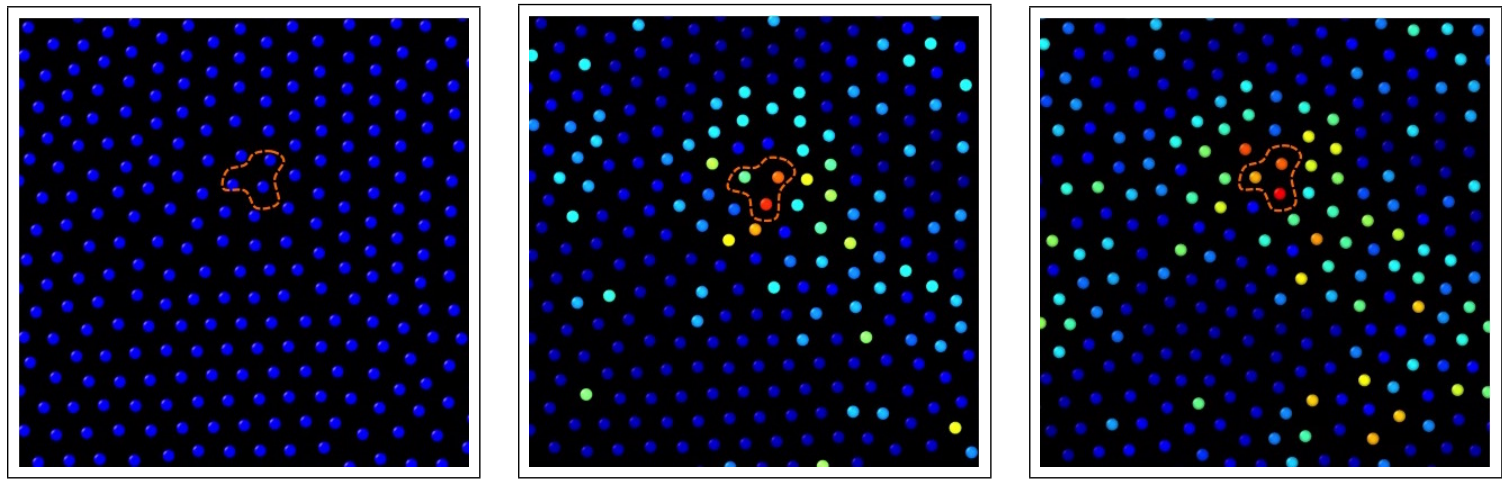

Figure 5.19: Evolution of a zone with high values of $\eta_{i}^{\text {Mises }}$ from $t_{1}$ to $t_{\max }$ to $\epsilon_{x x}=20 \%$ respectively.

The zone shown is the same as the one used in figure 5.18. Atoms are coloured according to their $\eta_{i}^{\text {Mises }}$ value, ranging from 0.02-0.2

5.20. There does not seem to be a great difference between the two prior $\eta_{i}^{\text {Mises }}$ figures (figures 5.17 and 5.20), other than an increase in the number of affected atoms (strain propagation) and an increase in the $\eta_{i}^{\text {Mises }}$ value for atoms that were already affected. 


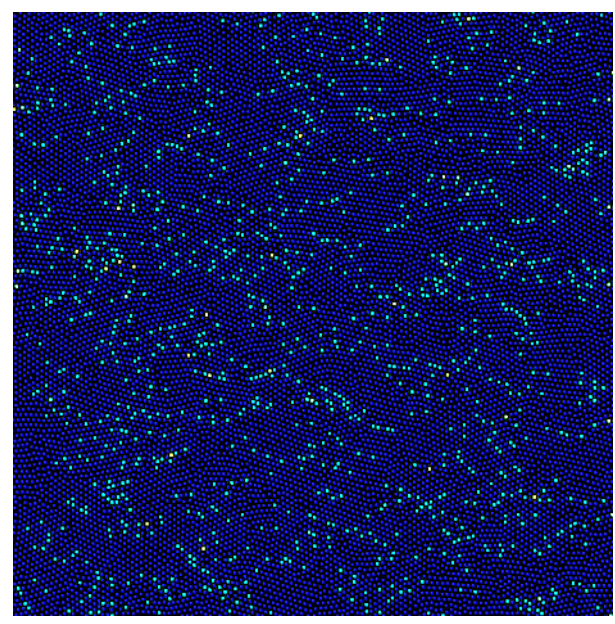

(a) True specimen $\eta_{i}^{\text {Mises }}{ }_{\max }=0.1341$

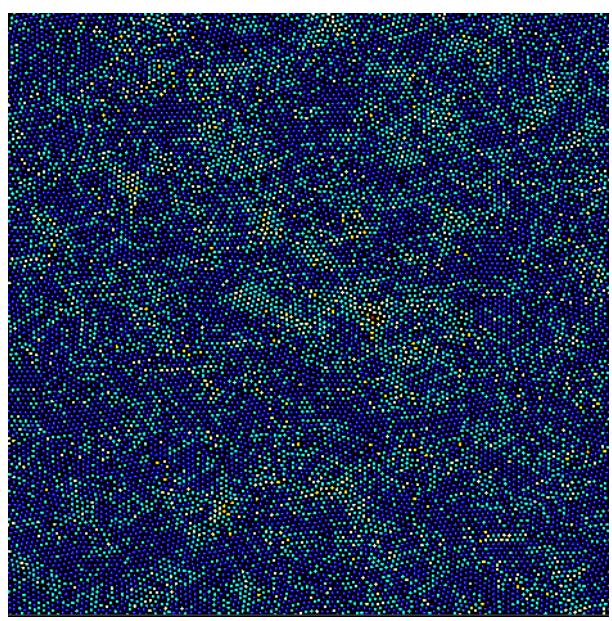

0.2

0.18

0.16

0.14

0.12

0.1

0.08

0.06

0.04

0.02

Figure 5.20: Intensity plots of $\eta_{i}^{\text {Mises }}$ for $t=t_{\max }$. Atoms are coloured according to their $\eta_{i}^{\text {Mises }}$ value, ranging from $0.02-0.2$

Timestep: $t$ when $\epsilon_{x x}=20 \%$

By the end of the deformation, the strain has been distributed across the entire specimen. Figure 5.21 shows the resulting $\eta_{i}^{\text {Mises }}$ for both specimens. The $\eta_{i}^{\text {Mises }}$ values have increased in both specimens and the affected areas have propagated. The somewhat even distribution of the strain and its propagation throughout the deformation make the initial affected areas hard to distinguish at the end.

In the Wang tiling specimen, some regions present high values of $\eta_{i}^{\text {Mises }}$, as seen in closeup figure 5.18. One of the concerns was if $\mathrm{BD}$ defects enabled the formation of STZs, the microscopic analysis shows that no STZs were created during the deformation. We can therefore conclude the $\mathrm{BD}$ defect does not enable the formation of STZs but might have an influence on strain distribution in the volumes. 


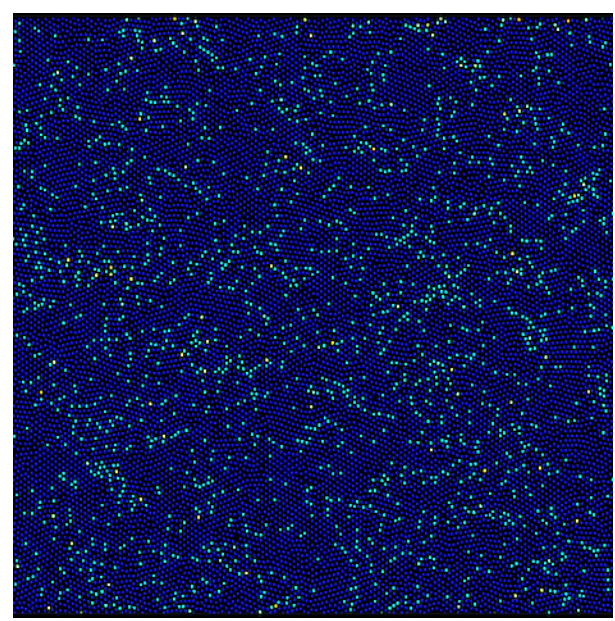

(a) True specimen

$$
\eta_{i}^{\text {Mises }}{ }_{\text {max }}=0.2886
$$

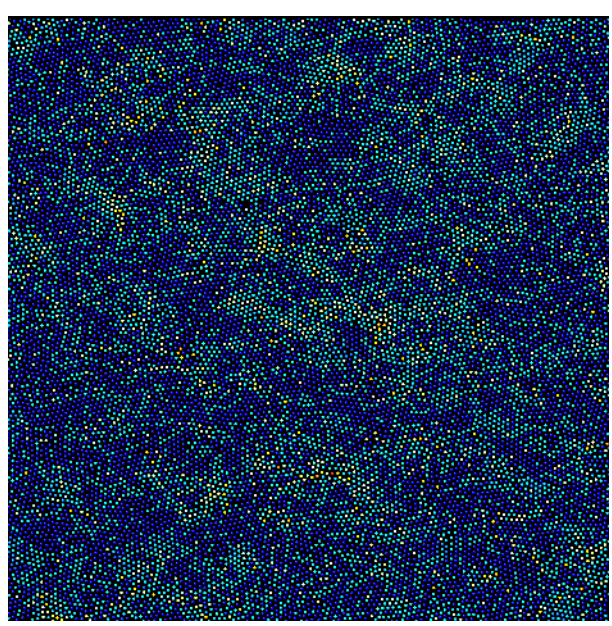

0.2

0.18

0.16

0.14

0.12

0.1

0.08

0.06

0.04

0.02

Figure 5.21: Intensity plots of $\eta_{i}^{\text {Mises }}$ for $\epsilon_{x x}=20 \%$. Atoms are coloured according to their $\eta_{i}^{\text {Mises }}$ value, ranging from $0.02-0.2$

\subsection{Results analysis}

In this chapter, clear differences between a random generated (true specimen) and semi-random generated (Wang tiling specimen) system were presented. While both specimens are composed of the same particles, $\mathrm{Cu}$ and $\mathrm{Zr}$, the system's mechanical responses are very different. The stress-strain reaction curve for both specimens is different; the two-dimensional true specimen presented a higher maximum strength than the Wang tiling specimen.

It is difficult to determine the causes of these differences from a macroscopic perspective. Stress-strain curves provide good information on how a specimen deforms. These graphs (figures 5.2 to 5.7) provided a good comparison on the deformation of both specimens and the differences in their strength.

In order to get a better understanding on the macroscopic differences of both specimens, two microscopic analysis were conducted on the formation of atomic bond deficiency defects (BD defects) and shear transformation zones (STZs). 
One feature of metallic glasses is the presence of BD defects. These defects are defined as gaps or holes in the system where the density is lower. BD defects are considered to be transport carriers of motion; Zhu et al. [63] found that they played an important role in motion diffusion and the formation of shear bands in a system.

A comparison on both specimens was conducted to determine the presence of BD defects and their influence on the stress-strain curve of each specimen. Wang tiling specimens presented this defect from the starting configuration $\left(t_{0}\right)$ and their concentration increased throughout the deformation process. The BD defect concentration for the Wang tiling specimen at the timesteps studied were always higher than in the true specimen. Considering they are motion carriers, the true specimen might have a higher maximum strength because it has a lower concentration of them.

The second analysis consisted in determining the formation of STZs in the system. The visualization of STZs was possible by calculating $\eta_{i}^{\text {Mises }}$ per atom and visualizing the differences in value through OVITO.

The conclusion from the deformation analysis is that there is a mechanical response difference between both specimens (which was quantified through the RMS). The true specimens are significantly stronger than the Wang tiling specimens. This difference can be attributed to a higher concentration of BD defects in the Wang tiling specimen that might condition the distribution of strain in the system. The resulting Wang tiling specimen cannot be considered representative of the true specimen as there are considerable differences in its mechanical response. Further investigation into methods of Wang tiling generation are needed to determine if better fidelity can be achieved with the tiled specimens. 


\section{Chapter 6}

\section{Conclusions}

This research consisted of the implementation of a mathematical model for the representation of random structures, known as Wang tiling. It has been used for the creation of non-periodic lattices in fields related to math, computer graphics, biology and to a limited extend, materials engineering. This work is an extension to the list of applications to include metallic glasses.

The objectives of this research were met by the establishment of a successful methodology for the creation of metallic glasses using Wang tilings, the implementation of the Wang tiling model in atomistic simulations and by the comparison between the Wang tiling specimen and the true specimen. However, the conclusion is that the resulting two-dimensional Wang tiling specimen was very different from the true specimen; the Wang tiling specimen cannot be considered a surrogate of the true specimen.

The current chapter will summarize the different tasks that were performed throughout the research, the results, and set the scene for future work. 


\subsection{Methodology findings}

Chapter 4 consisted of the establishment of two methodologies that create a random system and a semi-random system (true specimen and Wang tiling specimen respectively). The true specimen, considered a random system, originates from an initial random atomic configuration that is heated, quenched and minimized to equilibrium. The Wang tiling specimen on the other hand, considered a semi-random system, is formed from the assembly of Wang tiles constrained to meet edge code compatibilities. These Wang tiles are formed from the assembly of extracted regions of the true specimen.

\subsubsection{True specimen findings}

The first model created in this research was the random system, referred to as the true specimen. It consisted of the creation of a two-dimension two-component system that would later be used for the creation of the Wang tiling specimen.

The development of a true specimen consisted of four steps: (i) creation of a random initial configuration, (ii) energy minimization of the initial configuration, (iii) heating and quenching of the system, and (iv) energy minimization of the system after quenching. This specimen was created using the LAMMPS simulation package [33], and the result was the development of an equilibrated, two-dimensional, twocomponent $(\mathrm{Cu}-\mathrm{Zr})$, homogeneous metallic glass specimen.

The true specimen methodology can be improved if the initial configuration takes into account interatomic distances. This would reduce the need to have an energy minimization after the initial configuration is created which might save time. 


\subsubsection{Wang tiling specimen findings}

One of the key requirements for the creation of a Wang tiling specimen is the stability of the true specimen. Using a homogeneous and equilibrated true specimen leads to less issues during the Wang tiling specimen creation. The development of a Wang tiling specimen consisted on i) sample extraction from the true specimen, ii) creation of prototiles, iii) creation of Wang tiles and establishment of a tile set, and iv) assembly of Wang tiles for the creation of the tiling.

The Wang tiling specimen creation procedure presented several limitations that are great opportunities for future work. The two main findings were:

1. Prototiles and Wang tiles depend on the components used during the assembly. Edges with atoms in close proximity, overlapping or missing atoms, are challenges that need to be addressed. Several solutions are presented in section 4.2. An alternative to the proposed solutions can be a selective selection of samples. Sample extraction can be done taking into account the atomic density at the edges. These samples generated would not have a fixed geometry but would have a uniform density edge. This might decrease the issues found during assembling and save time reaching more stable structures. This alternative was not included in this research as edge issues were dealt with during the procedure and the prototiles and Wang tiles created are close to stability.

2. An energy minimization with constraints can be a good solution for ensuring periodicity in all prototiles. The energy minimizations performed in this research were done with groups of atoms (similar to molecules) rather than with individual atoms. This energy minimization was limited by the software use (LAMMPS).

The end result was the creation of a two-dimensional semi-random metallic glass 
(Wang tiling specimen), that was formed by 'stitching' images taken from the true specimen. The stability of the assembled Wang tiling specimen is limited by Wang tile edge issues during assembly. Edges of neighbouring tiles can present atoms in close proximity, overlapping or regions without atoms. These issues lead to regions with high energy that are more susceptible to plastic yield.

\subsection{Validation}

Once the true specimen and Wang tiling specimen were created, the next step was their comparison. Both specimens were submitted to uniform tension deformation and their reaction was analyzed and compared at the macroscopic and microscopic level.

At the macroscopic level, several systems for each specimen were created and the deviation of each population was determined. Wang tiling specimens presented high deviations between one another. The differences between specimens can be attributed to the use of different true specimens for their creation, edge issues that can be present in higher concentrations in some specimens than in others, and the presence of defects at the microscopic level. On the other hand, the true specimens created presented smaller variations amongst themselves.

A comparison between the true specimen and Wang tiling specimen showed a great deviation between them. The Wang tiling specimen has a lower maximum strength than the true specimen and yields plastically sooner. A possible explanation for this difference can be found at the microscopic level.

Two features were of interest at the microscopic level: the presence of atomic bond deficiency defects (BD defects) and the formation of shear transformation zones (STZs). The findings were a higher concentration of BD defects in the Wang tiling 
specimen since the start of the deformation and BD defect formation throughout the process. This high concentration can limit atom mobility and influences the distribution of shear throughout the system.

STZs were not identified during the deformation of the specimens. Rather, there is a homogenous distribution of strain across the volume. More atoms were affected by the deformation in the Wang tiling specimen than in the true specimen which explains the lower maximum strength.

In summary, this research presents a methodology for the creation of a twodimensional Wang tiling specimen and a true specimen, where the Wang tiling specimen is highly dependent on assembly issues. This specimen was less stable, presented more defects and reaches lower maximum strength values when submitted to uniform tension deformation.

The differences found in the macroscopic and microscopic analysis of this work might not be present if the Wang tiling specimen created were a better representation of a metallic glass. This can potentially be accomplished by: making more efficient sample extractions where edge densities are taken into account, yielding less edge related issues during the assembly of prototiles and the tiling, and by performing energy minimizations where an atom in a particular edge code (that is repeated several times in the tiling) would experience the same displacement throughout the entire tiling (ensuring Wang tile identities are identical after minimization).

Regardless of the differences found between the random system and the Wang tiling system, this work was a success as it adapted the Wang tiling model to atomistic simulations, created a successful methodology for the creation and implementation of Wang tilings, and determined the accuracy of the tiling system in comparison to a metallic glass. 


\section{List of References}

[1] R. Aga and J. Morris, Materials Science and Technology. 2008.

[2] Y. Waseda and T. Masumoto, "Structure of amorphous fe80-p13-c7 alloy by x-ray diffraction," Zeitschrift für Physik B Condensed Matter, vol. 22, no. 2, pp. 121-126, 1975.

[3] L. H. Van Vlack, Elements of Materials Science and Engineering. AddisonWesley Publishing Company, 6 ed., 1989.

[4] Fréchette, Non-crystalline solids. John Wiley \& Sons, Inc., 1958.

[5] W. Nowacki, T. Matsumoto, and A. Edenharter, "Classification of crystalline substances by crystal systems, crystal classes, bravais lattices and space groups," Acta crystallographica, vol. 22, no. 6, pp. 935-940, 1967.

[6] M. Ashby and A. Greer, "Metallic glasses as structural materials," Scripta Materialia, vol. 54, no. 3, pp. 321-326, 2006.

[7] B. J. Alder and T. E. Wainwright, "Studies in molecular dynamics. i. general method," The Journal of Chemical Physics, vol. 31, no. 2, 1959.

[8] H. Wang, "Proving theorems by pattern recognitionii," Bell system technical journal, vol. 40, no. 1, pp. 1-41, 1961.

[9] S. Elliot, Physics of Amorphous Materials. Longman Scientific and Technical, 2 ed., 1990.

[10] J. M. Ziman, Models of disorder: the theoretical physics of homogeneously disordered systems. CUP Archive, 1979.

[11] W. Klement, R. Willens, and P. Duwez, "Non-crystalline structure in solidified gold-silicon alloys," Nature, vol. 187, pp. 869-870, 1960. 
[12] M. Ashby and A. Greer, "Metallic glasses as structural materials," Scripta Materialia, vol. 54, no. 3, pp. 321-326, 2006.

[13] D. Turnbull and M. H. Cohen, "Free-volume model of the amorphous phase: glass transition," The Journal of chemical physics, vol. 34, no. 1, pp. 120-125, 1961.

[14] W.-H. Wang, C. Dong, and C. Shek, "Bulk metallic glasses," Materials Science and Engineering: R: Reports, vol. 44, no. 2, pp. 45-89, 2004.

[15] A. Takeuchi and A. Inoue, "Classification of bulk metallic glasses by atomic size difference, heat of mixing and period of constituent elements and its application to characterization of the main alloying element," Materials Transactions, vol. 46, no. 12, pp. 2817-2829, 2005.

[16] T.-W. Wu and F. Spaepen, "The relation between enbrittlement and structural relaxation of an amorphous metal," Philosophical Magazine B, vol. 61, no. 4, pp. 739-750, 1990.

[17] F. Shimizu, S. Ogata, and J. Li, "Theory of shear banding in metallic glasses and molecular dynamics calculations," Materials transactions, vol. 48, no. 11, pp. 2923-2927, 2007.

[18] G. Shen, M. L. Rivers, S. R. Sutton, N. Sata, V. B. Prakapenka, J. Oxley, and K. S. Suslick, "The structure of amorphous iron at high pressures to 67gpa measured in a diamond anvil cell," Physics of the Earth and Planetary Interiors, vol. 143, pp. 481-495, 2004.

[19] T. R. Anantharaman, "Metallic glasses: production, properties and applications," Trans Tech Publications SA, Trans Tech House, CH-4711 Aedermannsdorf, Switzerland, 1984. 302, 1984.

[20] A. Argon, "A theory for the low-temperature plastic deformation of glassy polymers," Philosophical Magazine, vol. 28, no. 4, pp. 839-865, 1973.

[21] V. Keryvin, "Indentation as a probe for pressure sensitivity of metallic glasses," Journal of Physics: Condensed Matter, vol. 20, no. 11, p. 114119, 2008.

[22] C. A. Schuh and A. C. Lund, "Atomistic basis for the plastic yield criterion of metallic glass," Nature materials, vol. 2, no. 7, pp. 449-452, 2003. 
[23] J. Lewandowski and A. Greer, "Temperature rise at shear bands in metallic glasses," Nature materials, vol. 5, no. 1, pp. 15-18, 2006.

[24] M. Falk and J. Langer, "Dynamics of viscoplastic deformation in amorphous solids," Physical Review E, vol. 57, no. 6, p. 7192, 1998.

[25] P. S. Steif, "Ductile versus brittle behavior of amorphous metals," Journal of the Mechanics and Physics of Solids, vol. 31, no. 5, pp. 359-388, 1983.

[26] Y. Li, Q. Guo, J. Kalb, and C. Thompson, "Matching glass-forming ability with the density of the amorphous phase," science, vol. 322, no. 5909, pp. 1816-1819, 2008.

[27] M. P. Allen and D. J. Tildesley, Computer simulation of liquids. Oxford university press, 1989.

[28] D. Frenkel and B. Smit, Understanding molecular simulation: from algorithms to applications, vol. 1. Academic press, 2001.

[29] K. Binder, J. Horbach, W. Kob, W. Paul, and F. Varnik, "Molecular dynamics simulations," Journal of Physics: Condensed Matter, vol. 16, no. 5, p. S429, 2004.

[30] B. Leimkuhler and C. Matthews, Molecular Dynamics: with deterministic and stochastic numerical methods, vol. 39. Springer, 2015.

[31] P. A. Dirac, "Quantum mechanics of many-electron systems," in Proceedings of the Royal Society of London A: Mathematical, Physical and Engineering Sciences, vol. 123, pp. 714-733, The Royal Society, 1929.

[32] E. B. Tadmor and R. E. Miller, Modeling materials: continuum, atomistic and multiscale techniques. Cambridge University Press, 2011.

[33] S. Plimpton, "Lammps users manual," Sandia National Laboratory, 2005.

[34] A. Stukowski, "Visualization and analysis of atomistic simulation data with ovitothe open visualization tool," Modelling and Simulation in Materials Science and Engineering, vol. 18, no. 1, p. 015012, 2010.

[35] M. Mendelev, M. Kramer, R. Ott, D. Sordelet, D. Yagodin, and P. Popel, "Development of suitable interatomic potentials for simulation of liquid and amorphous cu-zr alloys," Philosophical Magazine, vol. 89, no. 11, pp. 967-987, 2009. 
[36] J. Nocedal and S. J. Wright, "Conjugate gradient methods," Numerical Optimization, pp. 101-134, 2006.

[37] E. Bitzek, P. Koskinen, F. Gähler, M. Moseler, and P. Gumbsch, "Structural relaxation made simple," Physical review letters, vol. 97, no. 17, p. 170201, 2006.

[38] H. Ramalingam, M. Asta, A. Van de Walle, and J. Hoyt, "Atomic-scale simulation study of equilibrium solute adsorption at alloy solid-liquid interfaces," Interface Science, vol. 10, no. 2-3, pp. 149-158, 2002.

[39] H.-J. Lee, T. Cagin, W. L. Johnson, and W. A. Goddard III, "Criteria for formation of metallic glasses: The role of atomic size ratio," The Journal of chemical physics, vol. 119, no. 18, pp. 9858-9870, 2003.

[40] F. Albano and M. L. Falk, "Shear softening and structure in a simulated threedimensional binary glass," The Journal of chemical physics, vol. 122, no. 15, p. $154508,2005$.

[41] J. Rottler and M. O. Robbins, "Unified description of aging and rate effects in yield of glassy solids," Physical review letters, vol. 95, no. 22, p. 225504, 2005.

[42] J. Kari, "A small aperiodic set of wang tiles," Discrete Mathematics, vol. 160, no. 1, pp. 259-264, 1996.

[43] K. Culik II and J. Kari, "An aperiodic set of wang cubes," in J. UCS The Journal of Universal Computer Science, pp. 675-686, Springer, 1996.

[44] M. F. Cohen, J. Shade, S. Hiller, and O. Deussen, Wang tiles for image and texture generation, vol. 22. ACM, 2003.

[45] M. Doškář, Wang tiling for modelling of heterogeneous materials. PhD thesis, Czech Technical University in Prague, 2016.

[46] D. Shechtman, I. Blech, D. Gratias, and J. W. Cahn, "Metallic phase with long-range orientational order and no translational symmetry," Physical Review Letters, vol. 53, no. 20, p. 1951, 1984.

[47] D. Levine and P. J. Steinhardt, "Quasicrystals: a new class of ordered structures," Physical review letters, vol. 53, no. 26, p. 2477, 1984.

[48] R. Penrose, "The role of aesthetics in pure and applied mathematical research," Bulletin of the Institute of Mathematics and its Application, vol. 10, pp. 266-271, 1974. 
[49] L. Leuzzi and G. Parisi, "Thermodynamics of a tiling model," Journal of Physics A: Mathematical and General, vol. 33, no. 23, p. 4215, 2000.

[50] D. Aristoff and C. Radin, "First order phase transition in a model of quasicrystals," Journal of Physics A: Mathematical and Theoretical, vol. 44, no. 25, p. 255001, 2011.

[51] E. Winfree, F. Liu, L. A. Wenzler, and N. C. Seeman, "Design and self-assembly of two-dimensional dna crystals," Nature, vol. 394, no. 6693, pp. 539-544, 1998.

[52] H. Yan, S. H. Park, G. Finkelstein, J. H. Reif, and T. H. LaBean, "Dnatemplated self-assembly of protein arrays and highly conductive nanowires," Science, vol. 301, no. 5641, pp. 1882-1884, 2003.

[53] J. Novák, A. Kučerová, and J. Zeman, "Compressing random microstructures via stochastic wang tilings," Physical Review E, vol. 86, no. 4, p. 040104, 2012.

[54] A. A. Efros and W. T. Freeman, "Image quilting for texture synthesis and transfer," in Proceedings of the 28th annual conference on Computer graphics and interactive techniques, pp. 341-346, ACM, 2001.

[55] M. Doškář, J. Novák, and J. Zeman, "Aperiodic compression and reconstruction of real-world material systems based on wang tiles," Phys. Rev. E, vol. 90, p. 062118, Dec 2014.

[56] N. Mattern, J. Bednarčik, S. Pauly, G. Wang, J. Das, and J. Eckert, "Structural evolution of cu-zr metallic glasses under tension," Acta Materialia, vol. 57, no. 14, pp. 4133-4139, 2009.

[57] T. Fukunaga, K. Itoh, T. Otomo, K. Mori, M. Sugiyama, H. Kato, M. Hasegawa, A. Hirata, Y. Hirotsu, and A. Hannon, "Voronoi analysis of the structure of CuZr and NiZr metallic glasses," Intermetallics, vol. 14, no. 8-9, pp. 893-897, 2006.

[58] F. Spaepen, "Homogeneous flow of metallic glasses: A free volume perspective," Scripta materialia, vol. 54, no. 3, pp. 363-367, 2006.

[59] A. Lagae and P. Dutré, "An alternative for wang tiles: colored edges versus colored corners," ACM Transactions on Graphics (TOG), vol. 25, no. 4, pp. 14421459, 2006.

[60] M. Doškář, "Wang tilings for real world material systems," Master's thesis, Czech Technical University in Prague, Prague, 2013. 
[61] A. Zhu, G. Shiflet, and S. Poon, "Atomic bond deficiency as a structural defect in amorphous metals: Relevance to glass transitions," Acta Materialia, vol. 56, no. 3, pp. 593-601, 2008.

[62] A. Seeger, D. Schumacher, and W. Schilling, "Vacancies and interstitials in metals(proceedings of the international conference held at jülich, germany, 23-28 sept 1968)," 1970.

[63] A. Zhu, G. J. Shiflet, and S. J. Poon, "Atomic bond deficiency defects in amorphous metals," Metallurgical and Materials Transactions A, vol. 43, no. 10, pp. 3501-3509, 2012.

[64] Y. Limoge, "On the concept of free volume in amorphous materials," Scripta metallurgica et materialia, vol. 26, no. 5, pp. 809-812, 1992.

[65] F. Spaepen, R. Balian, M. Kléman, and J. Poirer, Defects in amorphous metals. North Holland Press, 1981. 